\title{
Application of 1,1-ADEQUATE, HMBC, and Density Functional Theory to Determine Regioselectivity in the Halogenation of Pyridine $\mathrm{N}$-Oxides
}

Tsang-Lin Hwang ${ }^{1, *}$, Michael D. Bartberger ${ }^{2,}$, and Ying Chen $^{3}$

${ }^{1}$ Attribute Sciences, ${ }^{2}$ Molecular Engineering, Therapeutic Discovery, and ${ }^{3}$ Drug Substance Technologies, Amgen Inc., One Amgen Center Drive, Thousand Oaks, CA 91320

*Corresponding authors: thwang@amgen.com; mbartber@amgen.com 


\section{Supporting information \\ Table of Contents}

S1. Experimental and Computational Details

Page 3

S2. Complete reference for the Gaussian 09 program system

Page 4

S3. Long range proton to carbon coupling constants in the pyridyl rings for compounds $\mathbf{2 ,}, \mathbf{3}, \mathbf{5}$ and 6

Page 5

S4. ${ }^{1} \mathrm{H}$ and ${ }^{13} \mathrm{C}$ NMR chemical shifts for the pyridyl rings of compounds $2,3,5,6,7$ to 20 and relevant regioisomers

Page 6

S5. 2D 1,1-ADEQUATE spectra for major compounds derived from $2, \mathbf{6}, \mathbf{1 3}, \mathbf{1 4}, \mathbf{1 5}, \mathbf{1 6}, \mathbf{1 7}, \mathbf{1 8}$, 19 and 20

Pages 7 to 16

S6. M06-2X/6-31+G(d,p) coordinates and energies of major and minor intermediates for species $2,6,7,9,11,13,15,17$, and 20

Pages 17 to 34

S7. M06-2X/6-31+G(d,p) coordinates and energies of major and minor halogenated products for species $2,6,7,9,11,13,15,17$, and 20

Pages 35 to 52

S8. Electrostatic potential-derived charges for substrate 7 and resultant major and minor deprotonated intermediates

Pages 53 to 56

S9. Product ratios and relative free energies of intermediates in Table 2, $\mathrm{CH}_{2} \mathrm{Cl}_{2}$ solvent

Page 57

S10. IEFPCM-M06-2X/6-31+G(d,p) coordinates and energies of major and minor intermediates for species $2,6,7,9,11,13,15,17$, and $20, \mathrm{CH}_{2} \mathrm{Cl}_{2}$ solvent

Pages 58 to 75 
S1. Experimental and Computational Details

All compounds in this study were synthesized and reported in ref. 13. Samples were dissolved into either $99.8 \%$ deuterated $\mathrm{DMSO}^{-\mathrm{d}_{6}}$ or $\mathrm{CDCl}_{3}$ (Cambridge Isotope Laboratories, Andover, Mass, USA), and placed into $5 \mathrm{~mm}$ NMR tubes. The NMR spectra were acquired on a Bruker ACANCE III $600 \mathrm{MHz}$ NMR spectrometer equipped with a $5 \mathrm{~mm} \mathrm{CPTCl}$ cryoprobe at 27 or $30^{\circ} \mathrm{C}$ (Bruker BioSpin Corporation, Billerica, Mass, USA). Standard 1D and 2D pulse sequences in the Bruker library were used for the study. Bruker's TopSpin and ACD (Advanced Chemical Development, Toronto, Canada) software were used for data processing. Chemical shifts were in the $\delta$ scale (ppm) by assigning the TMS proton and carbon peaks to $0 \mathrm{ppm}$. If TMS is not in the solvent, then assign residual solvent proton peak to $2.50 \mathrm{ppm}$ for $\mathrm{CD}_{2} \mathrm{HSOCD}_{3}$ or 7.27 ppm for $\mathrm{CHCl}_{3}$, and solvent carbon peak to $39.5 \mathrm{ppm}$ for the methyl group of DMSO or 77.0 ppm for $\mathrm{CDCl}_{3}$.

Density Functional Theory Calculations:

All calculations were performed with the Gaussian 09 program system. An initial conformational ensemble for each of the major and minor deprotonation intermediates were subject to geometry optimization on the $M 06-2 X / 6-31+G(d, p)$ energy surface, followed by harmonic frequency calculation for purposes of stationary point characterization and free energy determination. Vibrational frequencies and resultant thermochemical analyses were unscaled. Computational investigation of solvent effects utilized the IEFPCM implicit dichloromethane solvent model. 
S2. Complete reference for the Gaussian 09 program system:

Gaussian 09, Revision D.01, Frisch, M. J.; Trucks, G. W.; Schlegel, H. B.; Scuseria, G. E.; Robb, M. A.; Cheeseman, J. R.; Scalmani, G.; Barone, V.; Mennucci, B.; Petersson, G. A.; Nakatsuji, H.; Caricato, M.; Li, X.; Hratchian, H. P.; Izmaylov, A. F.; Bloino, J.; Zheng, G.; Sonnenberg, J. L.; Hada, M.; Ehara, M.; Toyota, K.; Fukuda, R.; Hasegawa, J.; Ishida, M.; Nakajima, T.; Honda, Y.; Kitao, O.; Nakai, H.; Vreven, T.; Montgomery, J. A., Jr.; Peralta, J. E.; Ogliaro, F.; Bearpark, M.; Heyd, J. J.; Brothers, E.; Kudin, K. N.; Staroverov, V. N.; Kobayashi, R.; Normand, J.; Raghavachari, K.; Rendell, A.; Burant, J. C.; Iyengar, S. S.; Tomasi, J.; Cossi, M.; Rega, N.; Millam, J. M.; Klene, M.; Knox, J. E.; Cross, J. B.; Bakken, V.; Adamo, C.; Jaramillo, J.; Gomperts, R.; Stratmann, R. E.; Yazyev, O.; Austin, A. J.; Cammi, R.; Pomelli, C.; Ochterski, J. W.; Martin, R. L.; Morokuma, K.; Zakrzewski, V. G.; Voth, G. A.; Salvador, P.; Dannenberg, J. J.; Dapprich, S.; Daniels, A. D.; Farkas, Ö.; Foresman, J. B.; Ortiz, J. V.; Cioslowski, J.; Fox, D. J. Gaussian, Inc., Wallingford CT, 2009. 
S3. Long range proton to carbon coupling constants in the pyridyl rings for compounds $2,3,5$ and 6 .

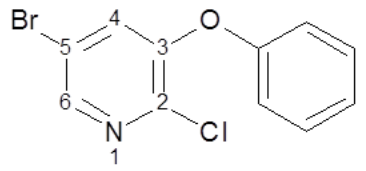

2

$$
\begin{aligned}
& { }^{3} \jmath_{\mathrm{H} 4 \mathrm{C} 2}=7.3 \mathrm{~Hz} \\
& { }^{2} \mathrm{~J}_{\mathrm{H} 4 \mathrm{C} 3}=3.9 \mathrm{~Hz} \\
& { }^{2} \mathrm{~J}_{\mathrm{H} 4 \mathrm{C} 5}=4.4 \mathrm{~Hz} \\
& { }^{3} \mathrm{~J}_{\mathrm{H} 4 \mathrm{C6}}=5.9 \mathrm{~Hz} \\
& { }^{3} \mathrm{~J}_{\mathrm{HGC}}=13.4 \mathrm{~Hz} \\
& { }^{4} \mathrm{~J}_{\mathrm{H} 6 \mathrm{C} 3}=1.9 \mathrm{~Hz} \\
& { }^{3} \mathrm{~J}_{\mathrm{H6C4}}=5.6 \mathrm{~Hz} \\
& { }^{2} \mathrm{~J}_{\mathrm{H} 6 \mathrm{C} 5}=5.5 \mathrm{~Hz}
\end{aligned}
$$

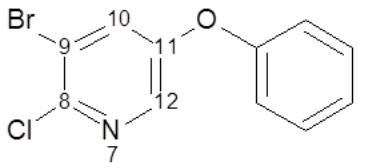

3

${ }^{3} \mathrm{~J}_{\mathrm{H} 10 \mathrm{C} 8}=8.2 \mathrm{~Hz}$

${ }^{2} \mathrm{~J}_{\mathrm{H} 10 \mathrm{CO}}=4.3 \mathrm{~Hz}$

$2 \mathrm{~J}_{\mathrm{H} 10 \mathrm{C} 11}=4.0 \mathrm{~Hz}$

${ }^{3} \mathrm{~J}_{\mathrm{H} 10 \mathrm{C} 12}=5.0 \mathrm{~Hz}$

${ }^{3} \mathrm{~J}_{\mathrm{H} 12 \mathrm{C} 8}=13.7 \mathrm{~Hz}$

${ }^{4} \mathrm{~J}_{\mathrm{H} 12 \mathrm{C} 9}=1.9 \mathrm{~Hz}$

${ }^{3} \mathrm{~J}_{\mathrm{H} 12 \mathrm{C} 10}=4.6 \mathrm{~Hz}$

${ }^{2} \mathrm{~J}_{\mathrm{H} 12 \mathrm{C} 11}=3.7 \mathrm{~Hz}$
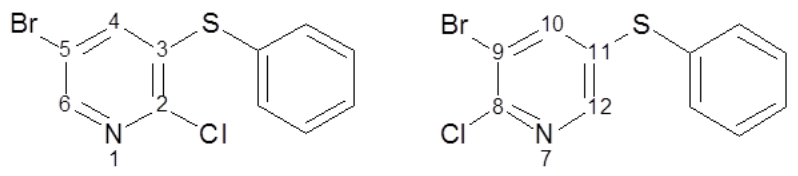

5

6

${ }^{3} \mathrm{~J}_{\mathrm{H} 4 \mathrm{C2}}=8.8 \mathrm{~Hz}$

${ }^{3} \mathrm{~J}_{\mathrm{H} 1 \mathrm{OC} 8}=8.1 \mathrm{~Hz}$

${ }^{2} \mathrm{~J}_{\mathrm{H} 4 \mathrm{C3}}=2.0 \mathrm{~Hz}$

${ }^{2} \mathrm{~J}_{\mathrm{H} 10 \mathrm{CO} 9}=3.6 \mathrm{~Hz}$

${ }^{2} \mathrm{~J}_{\mathrm{H} 4 \mathrm{CS}}=3.7 \mathrm{~Hz}$

${ }^{2} \mathrm{~J}_{\mathrm{H} 10 \mathrm{C} 11}=1.9 \mathrm{~Hz}$

${ }^{3} \mathrm{~J}_{\mathrm{H} 4 \mathrm{CC}}=5.8 \mathrm{~Hz}$

${ }^{3} \mathrm{~J}_{\mathrm{H} 10 \mathrm{C} 12}=6.5 \mathrm{~Hz}$

${ }^{3} \mathrm{~J}_{\mathrm{H} 6 \mathrm{C} 2}=13.7 \mathrm{~Hz}$

${ }^{3} \mathrm{~J}_{\mathrm{H} 12 \mathrm{C8}}=13.9 \mathrm{~Hz}$

${ }^{4} \mathrm{~J}_{\mathrm{H} 6 \mathrm{CC} 3}=1.7 \mathrm{~Hz}$

${ }^{4} \mathrm{~J}_{\mathrm{H} 12 \mathrm{C} 9}=1.8 \mathrm{~Hz}$

${ }^{3} \mathrm{~J}_{\mathrm{H} 6 \mathrm{C} 4}=5.2 \mathrm{~Hz}$

${ }^{3} \mathrm{~J}_{\mathrm{H} 12 \mathrm{C} 10}=5.6 \mathrm{~Hz}$

${ }^{2} \mathrm{~J}_{\mathrm{H} 6 \mathrm{C} 5}=5.1 \mathrm{~Hz}$

${ }^{2} \mathrm{~J}_{\mathrm{H} 12 \mathrm{C} 11}=6.8 \mathrm{~Hz}$ 
S4. ${ }^{1} \mathrm{H}$ and ${ }^{13} \mathrm{C}$ NMR chemical shifts for the pyridyl rings of compounds $2,3,5,6,7$ to 20 and relevant regioisomers.

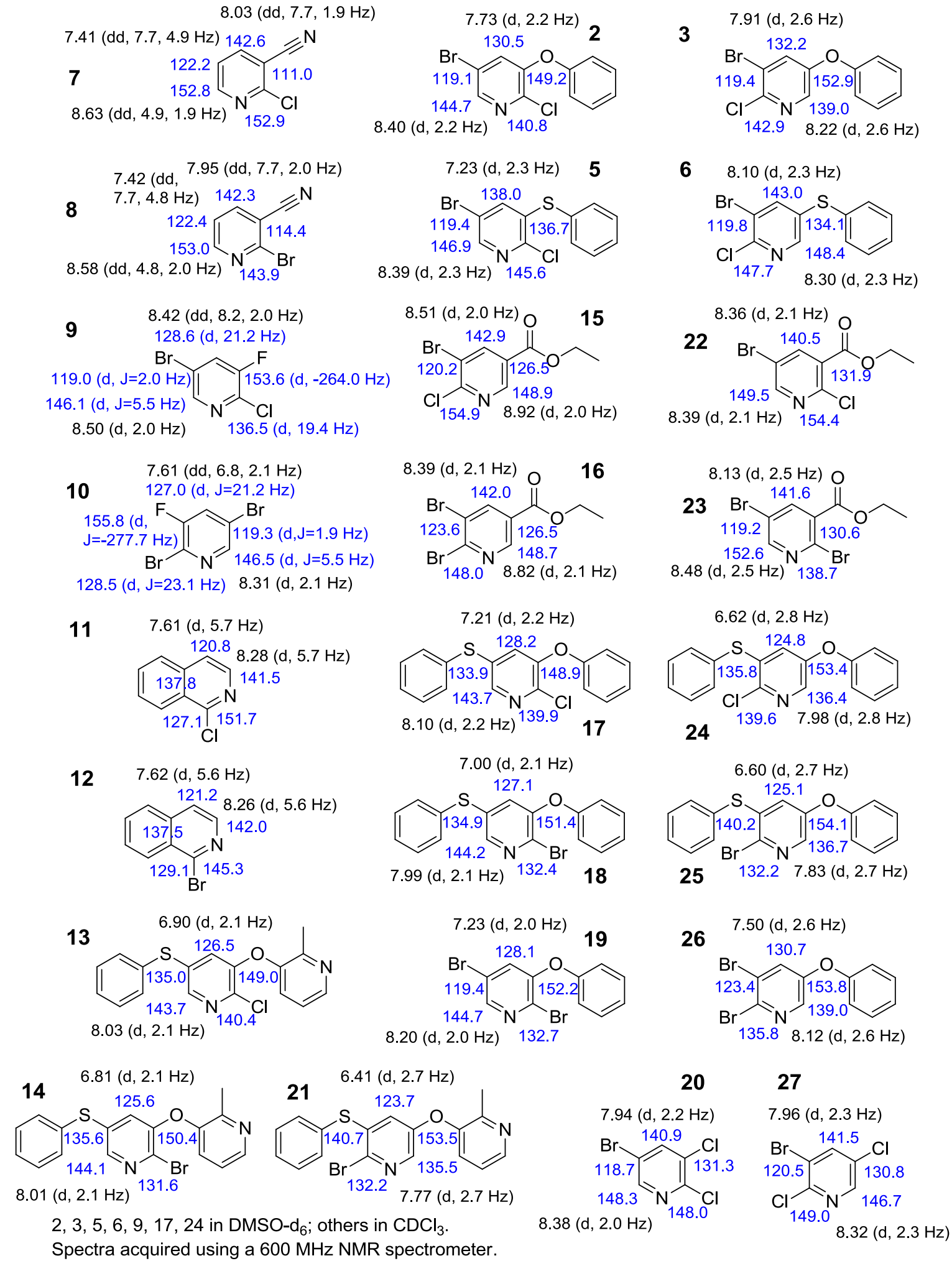


S5. 2D 1,1-ADEQUATE spectra for major compounds derived from $\mathbf{2}, \mathbf{6}, \mathbf{1 3}, \mathbf{1 4}, \mathbf{1 5}, \mathbf{1 6}$, $17,18,19$ and 20

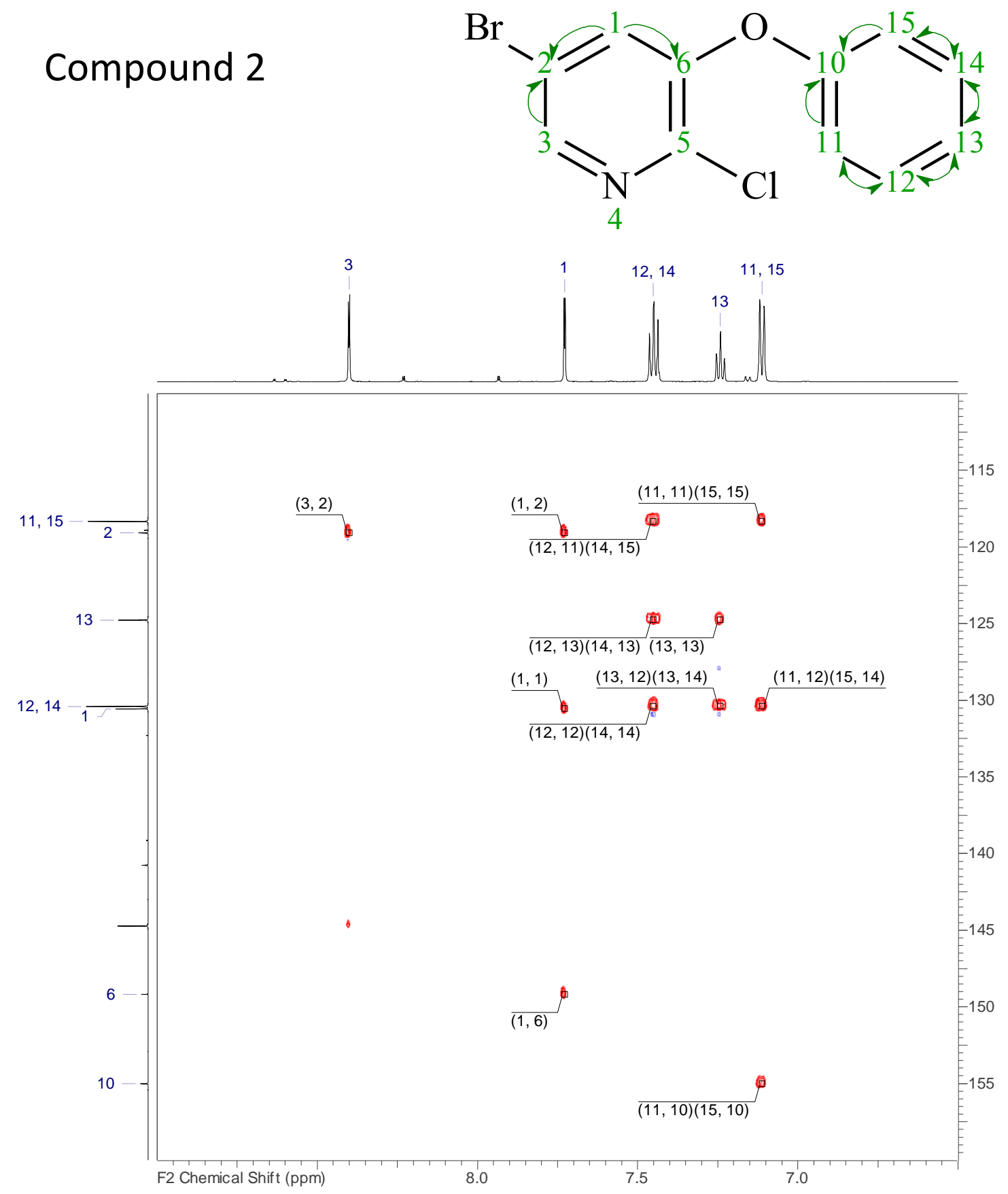

Compound 2 in DMSO- $\mathrm{d}_{6}$; 2D 1,1-ADEQUATE spectrum acquired using a $600 \mathrm{MHz}$ NMR spectrometer. 

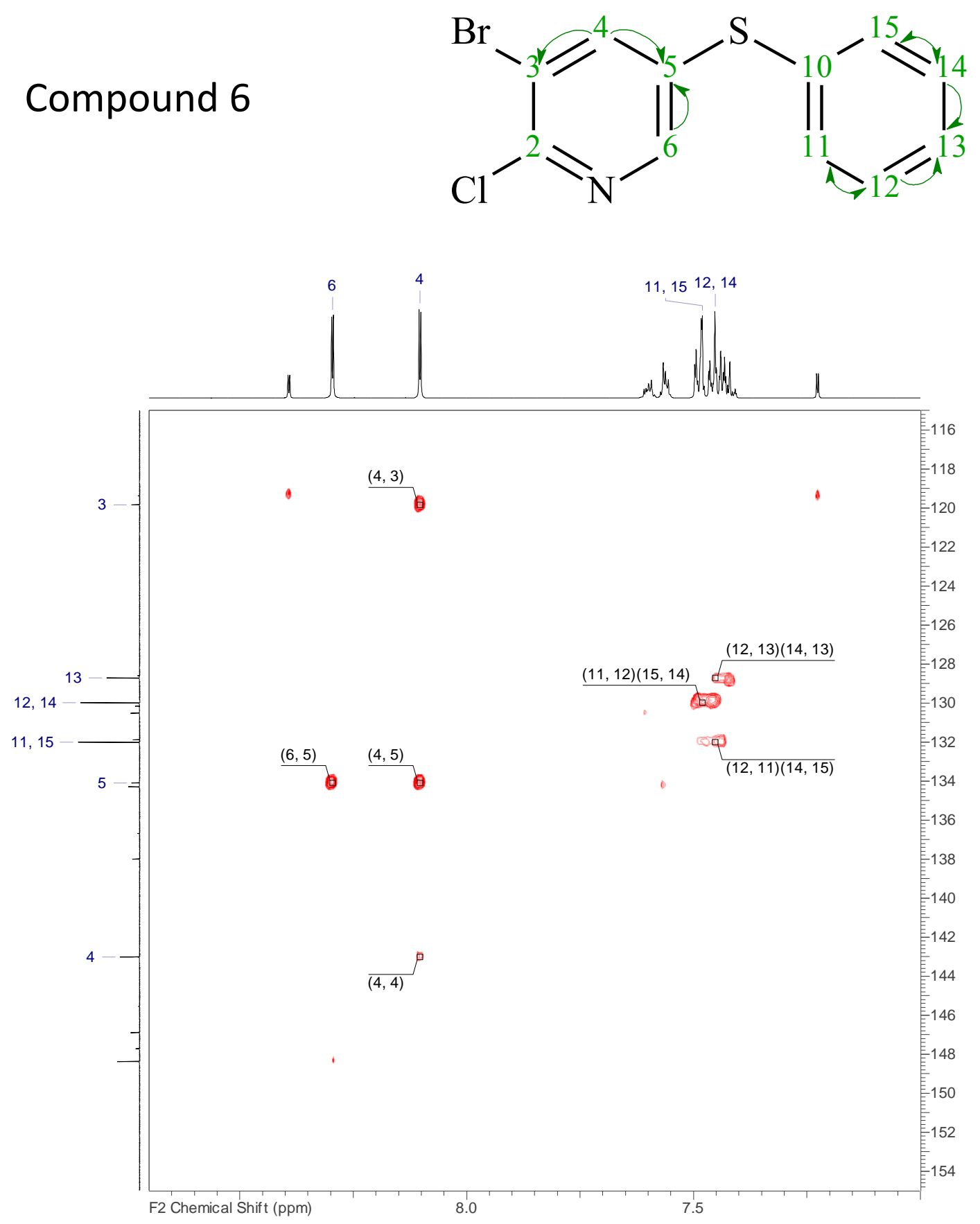

Compound 6 in DMSO- $\mathrm{d}_{6}$; 2D 1,1-ADEQUATE spectrum acquired using a $600 \mathrm{MHz}$ NMR spectrometer. 


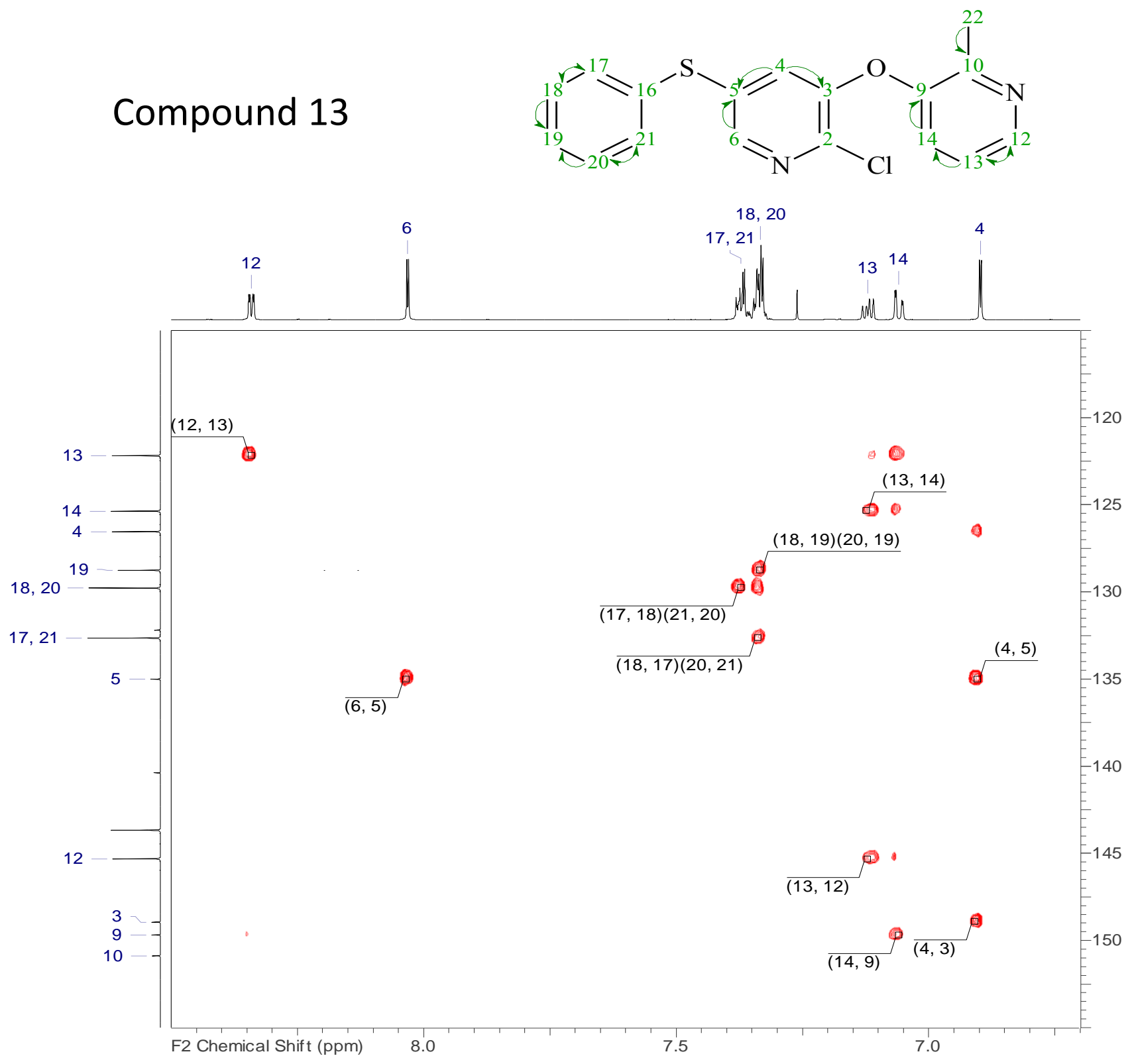

Compound 13 in $\mathrm{CDCl}_{3}$; 2D 1,1-ADEQUATE spectrum acquired using a $600 \mathrm{MHz} \mathrm{NMR}$ spectrometer. 


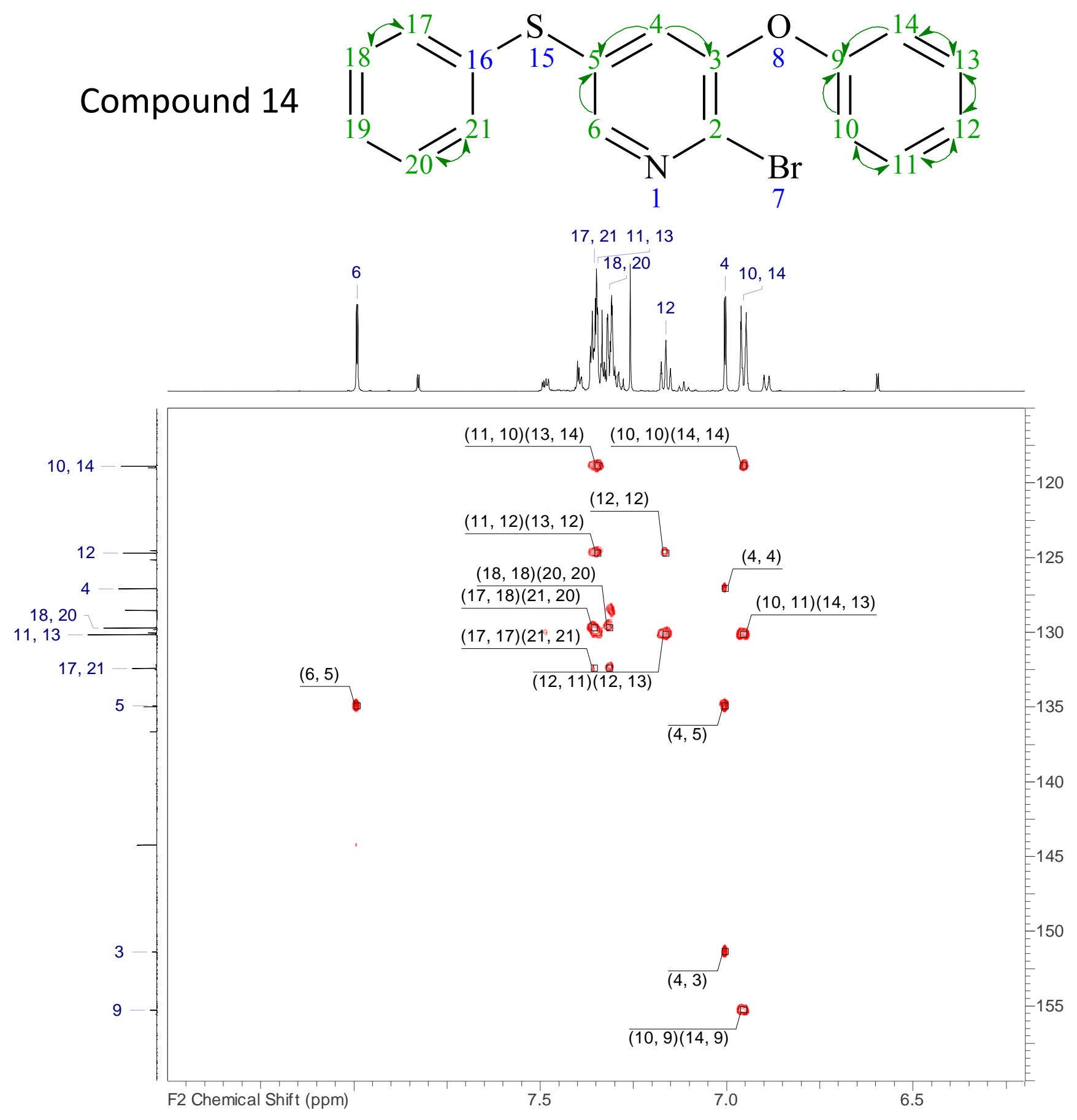

Compound 14 in $\mathrm{CDCl}_{3}$; 2D 1,1-ADEQUATE spectrum acquired using a $600 \mathrm{MHz} \mathrm{NMR}$ spectrometer. 


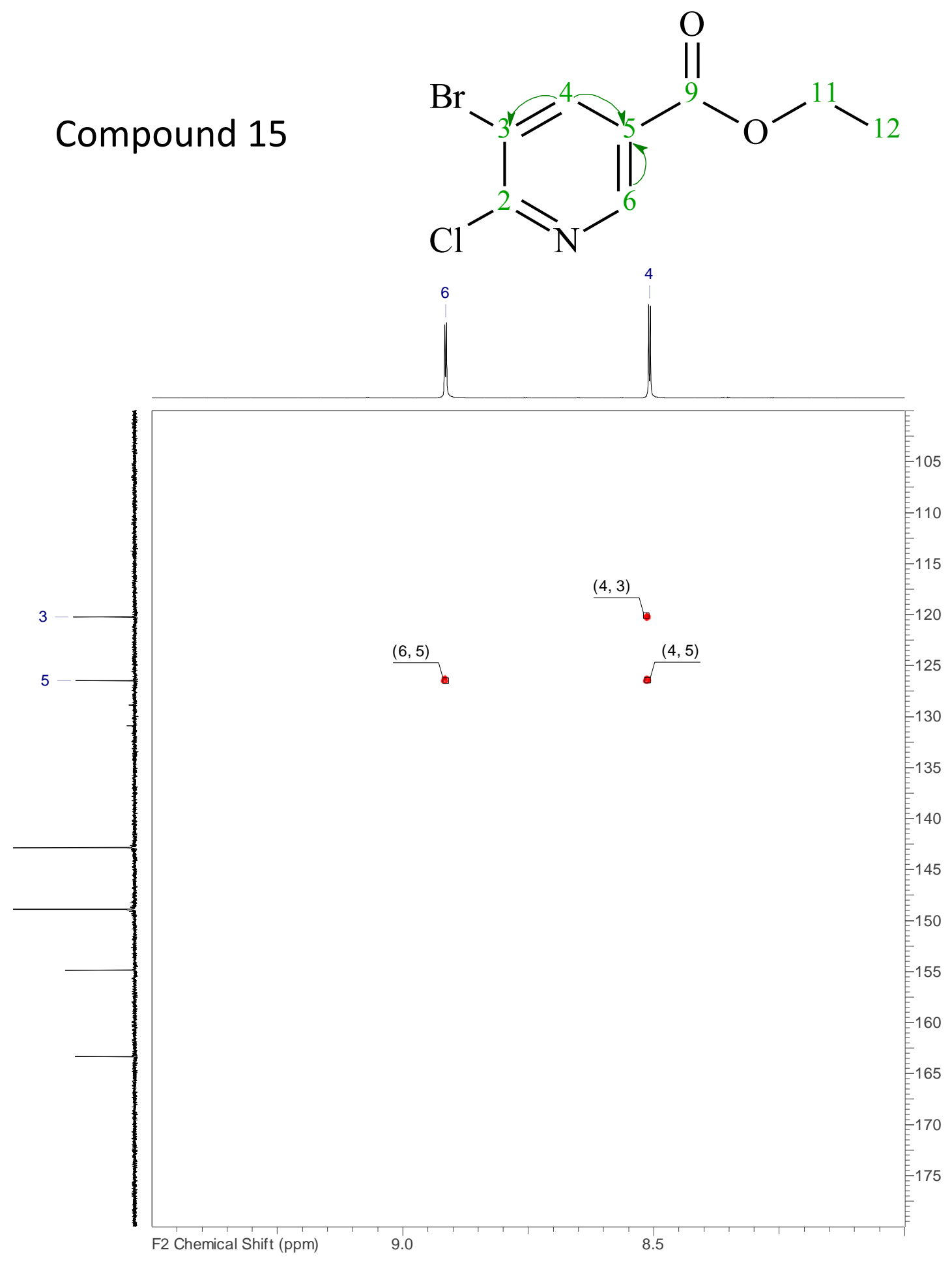

Compound 15 in $\mathrm{CDCl}_{3}$; 2D 1,1-ADEQUATE spectrum acquired using a $600 \mathrm{MHz} \mathrm{NMR}$ spectrometer. 


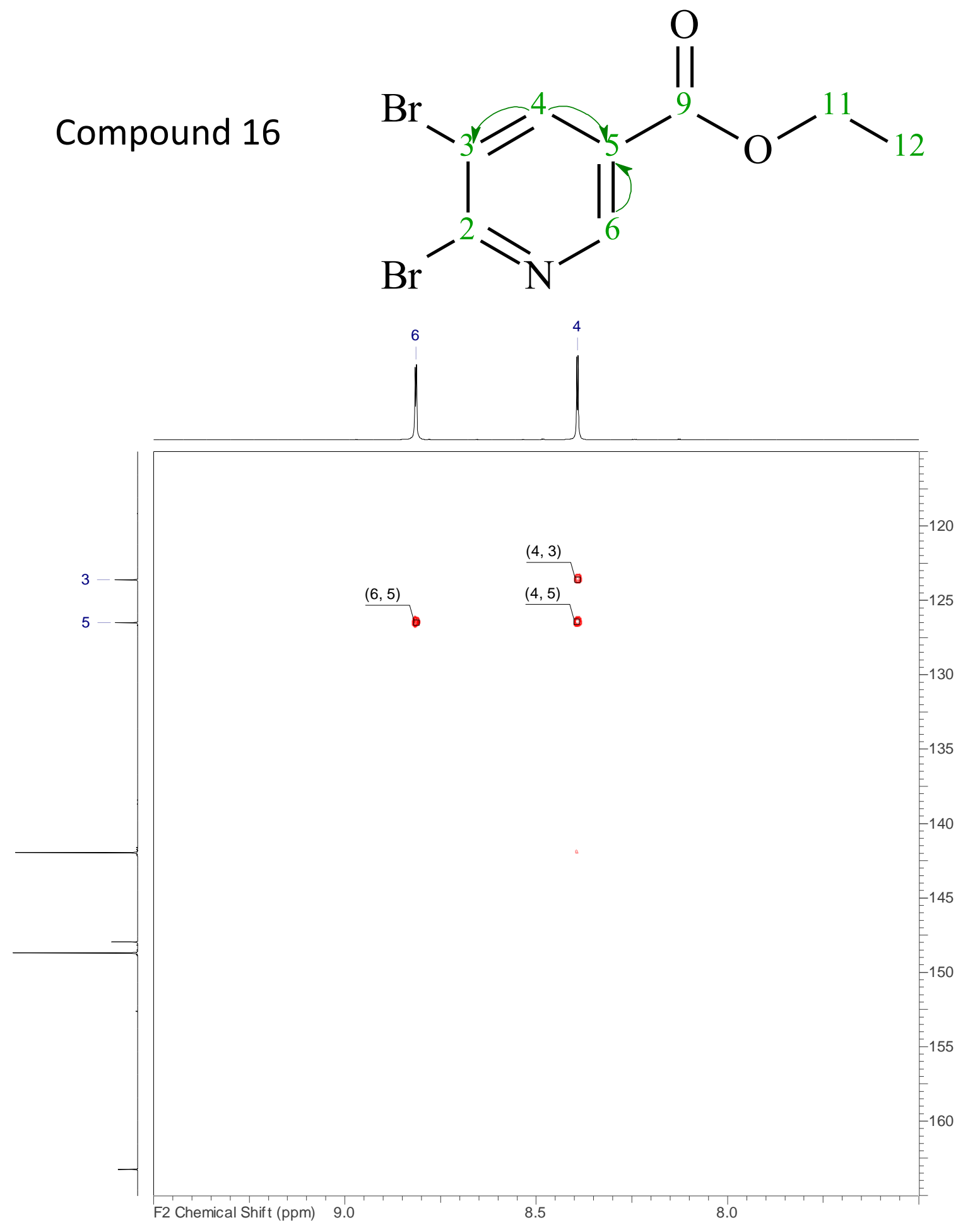

Compound 16 in $\mathrm{CDCl}_{3} ; 2 \mathrm{D}$ 1,1-ADEQUATE spectrum acquired using a $600 \mathrm{MHz} \mathrm{NMR}$ spectrometer. 


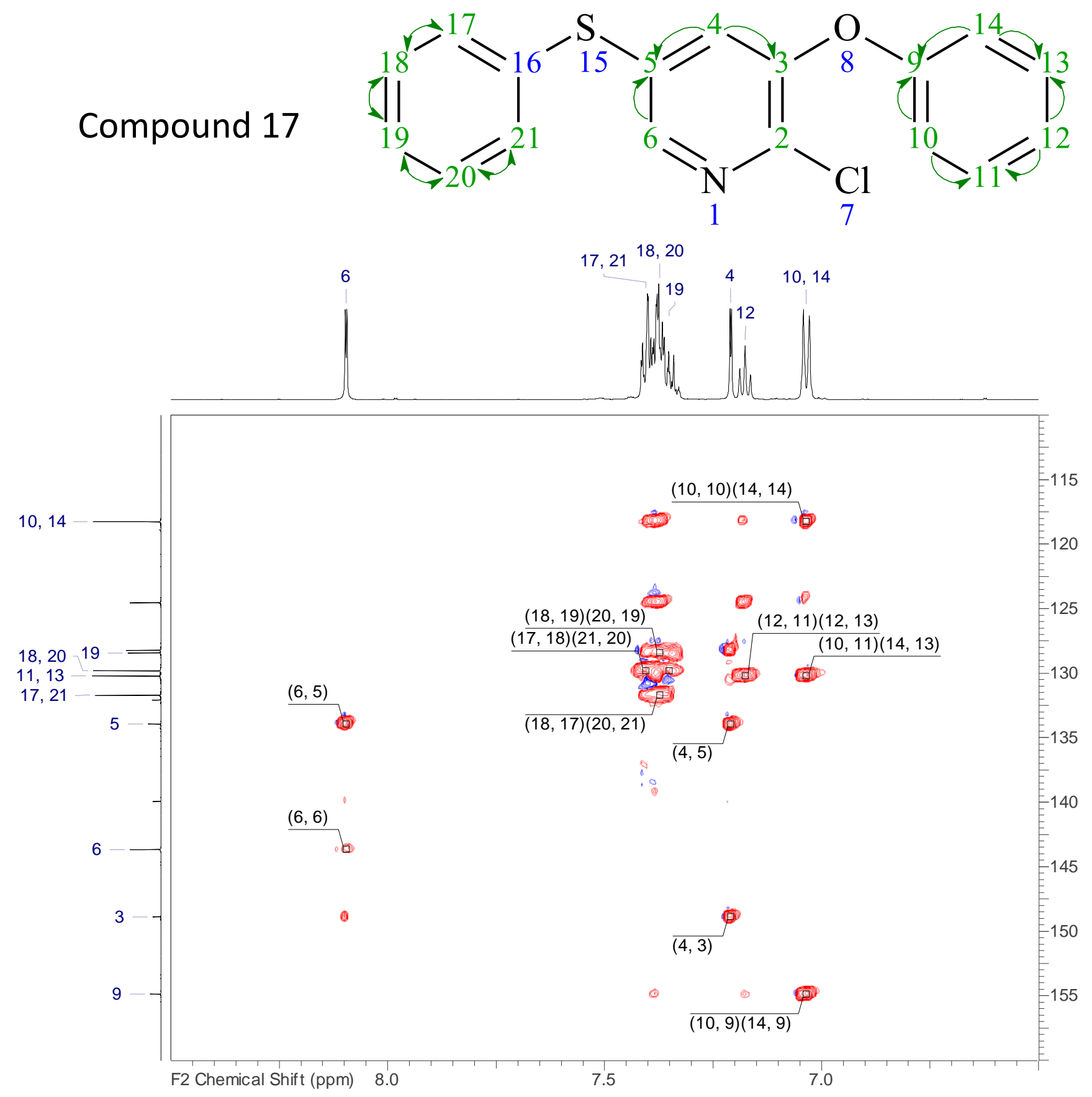

Compound 17 in DMSO- $\mathrm{d}_{6}$; 2D 1,1-ADEQUATE spectrum acquired using a $600 \mathrm{MHz}$ NMR spectrometer. 


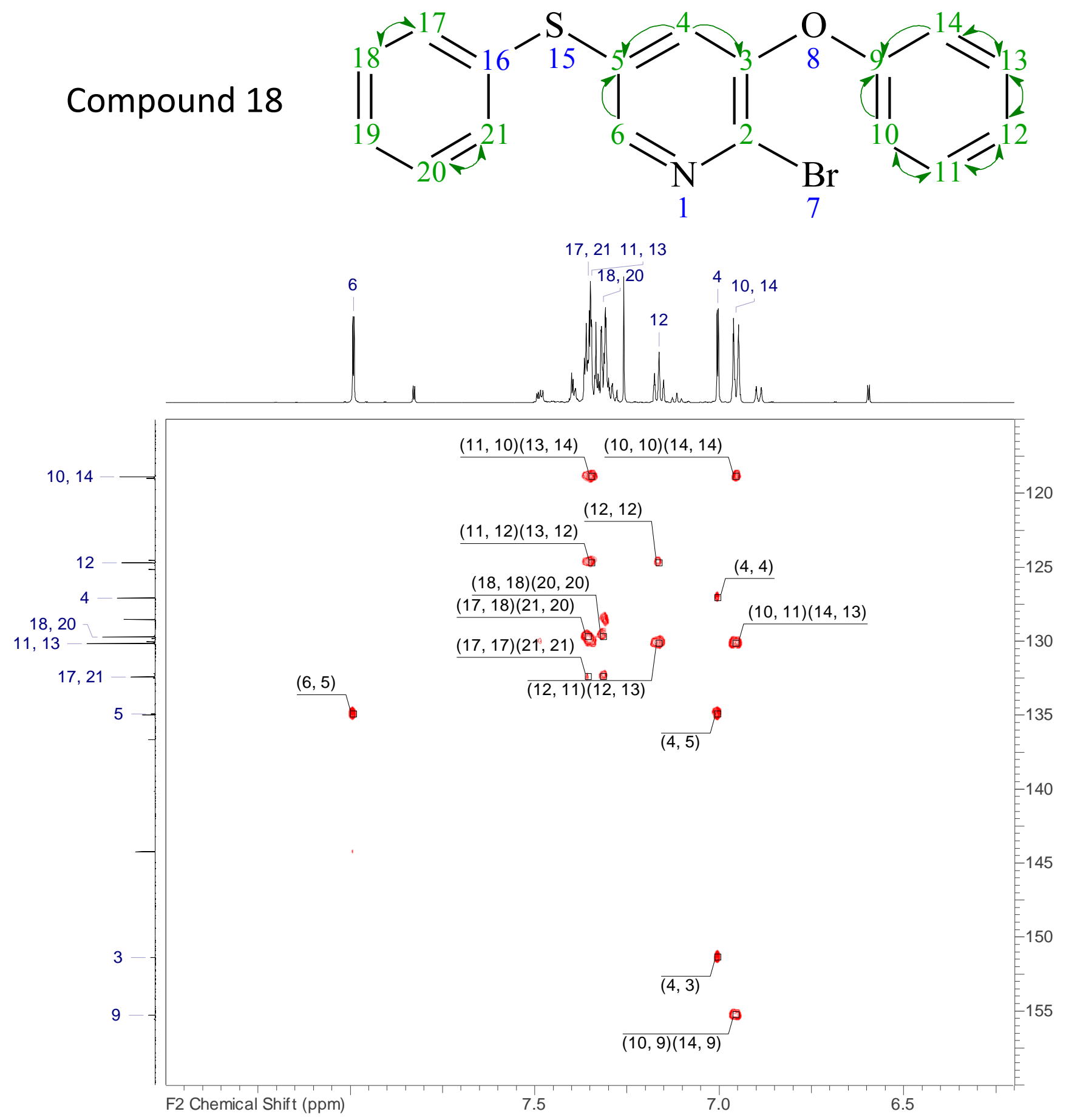

Compound 18 in $\mathrm{CDCl}_{3} ; 2 \mathrm{D}$ 1,1-ADEQUATE spectrum acquired using a $600 \mathrm{MHz} \mathrm{NMR}$ spectrometer. 


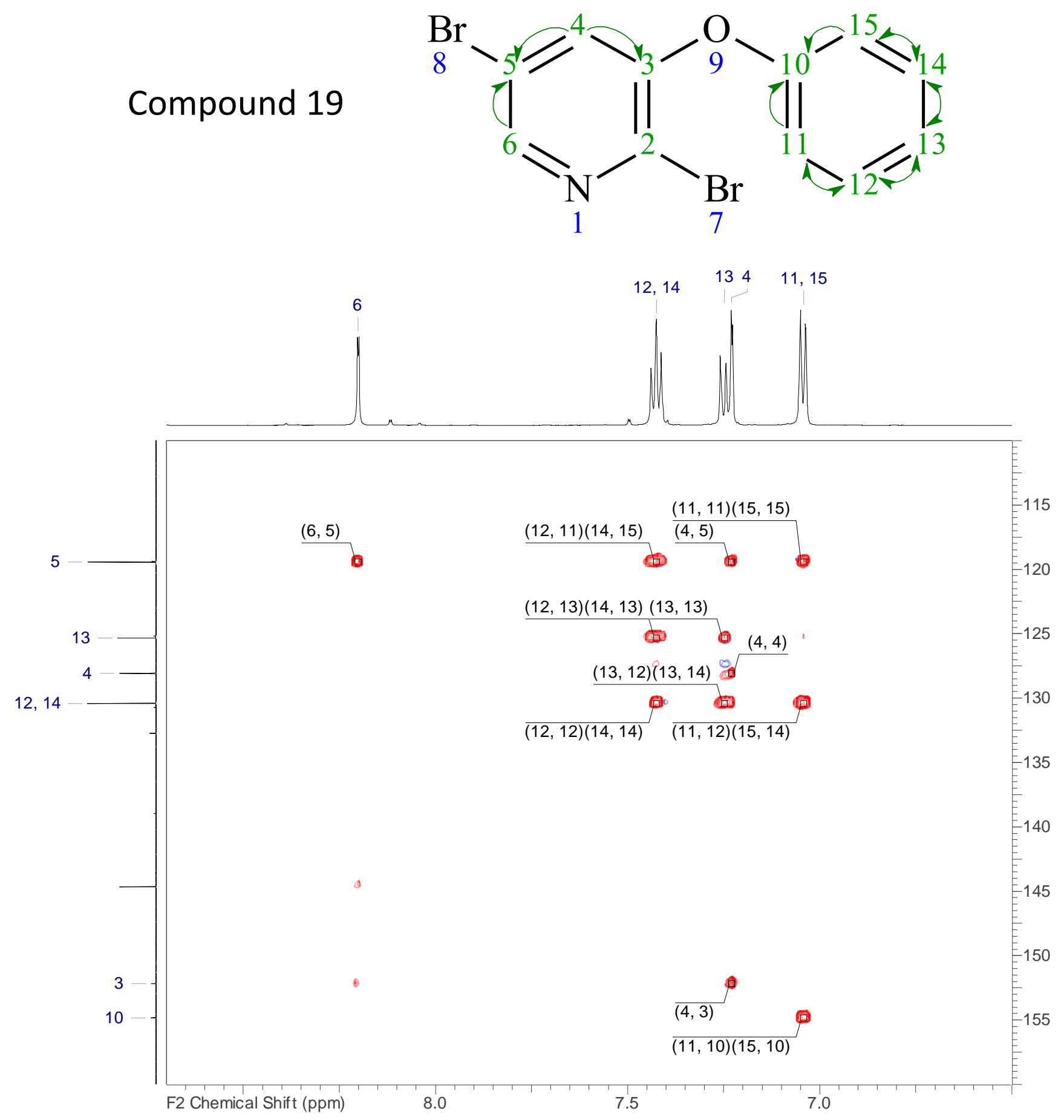

Compound 19 in $\mathrm{CDCl}_{3}$; 2D 1,1-ADEQUATE spectrum acquired using a $600 \mathrm{MHz} \mathrm{NMR}$ spectrometer. 
Compound 20
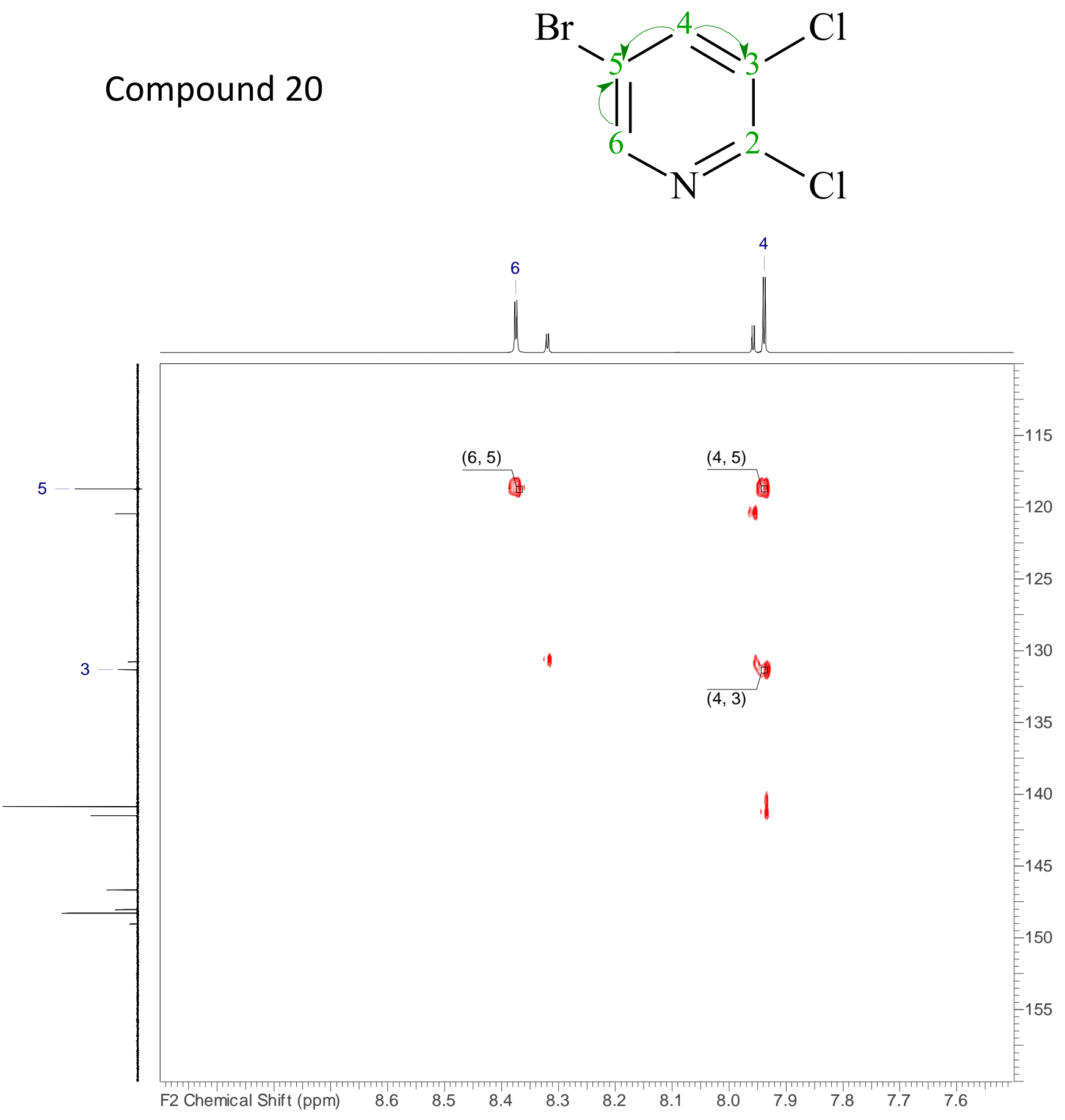

Compound 20 in $\mathrm{CDCl}_{3}$; 2D 1,1-ADEQUATE spectrum acquired using a $600 \mathrm{MHz} \mathrm{NMR}$ spectrometer. 


\section{S6. M06-2X/6-31+G(d,p) coordinates and energies of major and minor intermediates for species 2, 6, 7, 9, 11, 13, 15, 17, and 20}

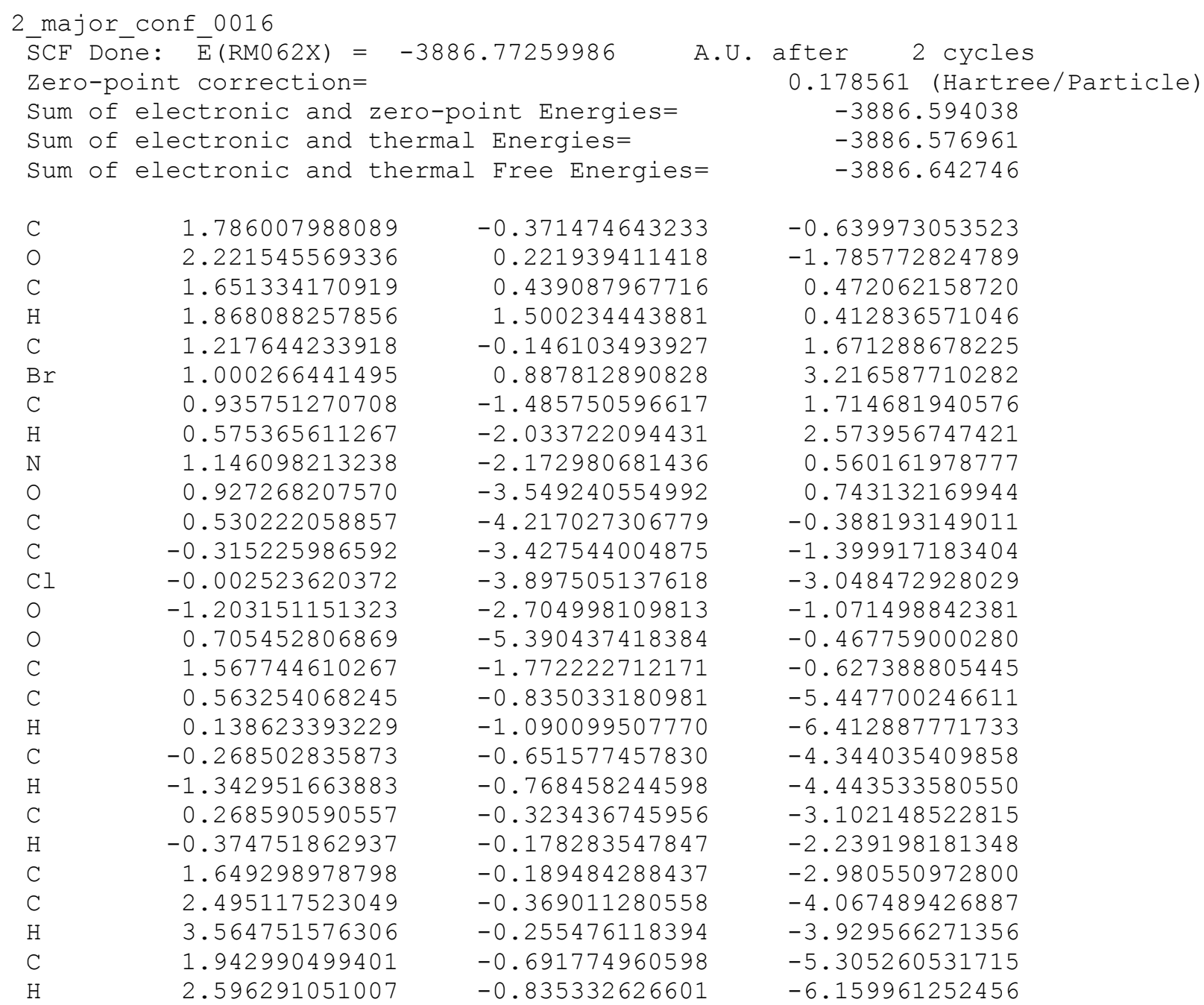




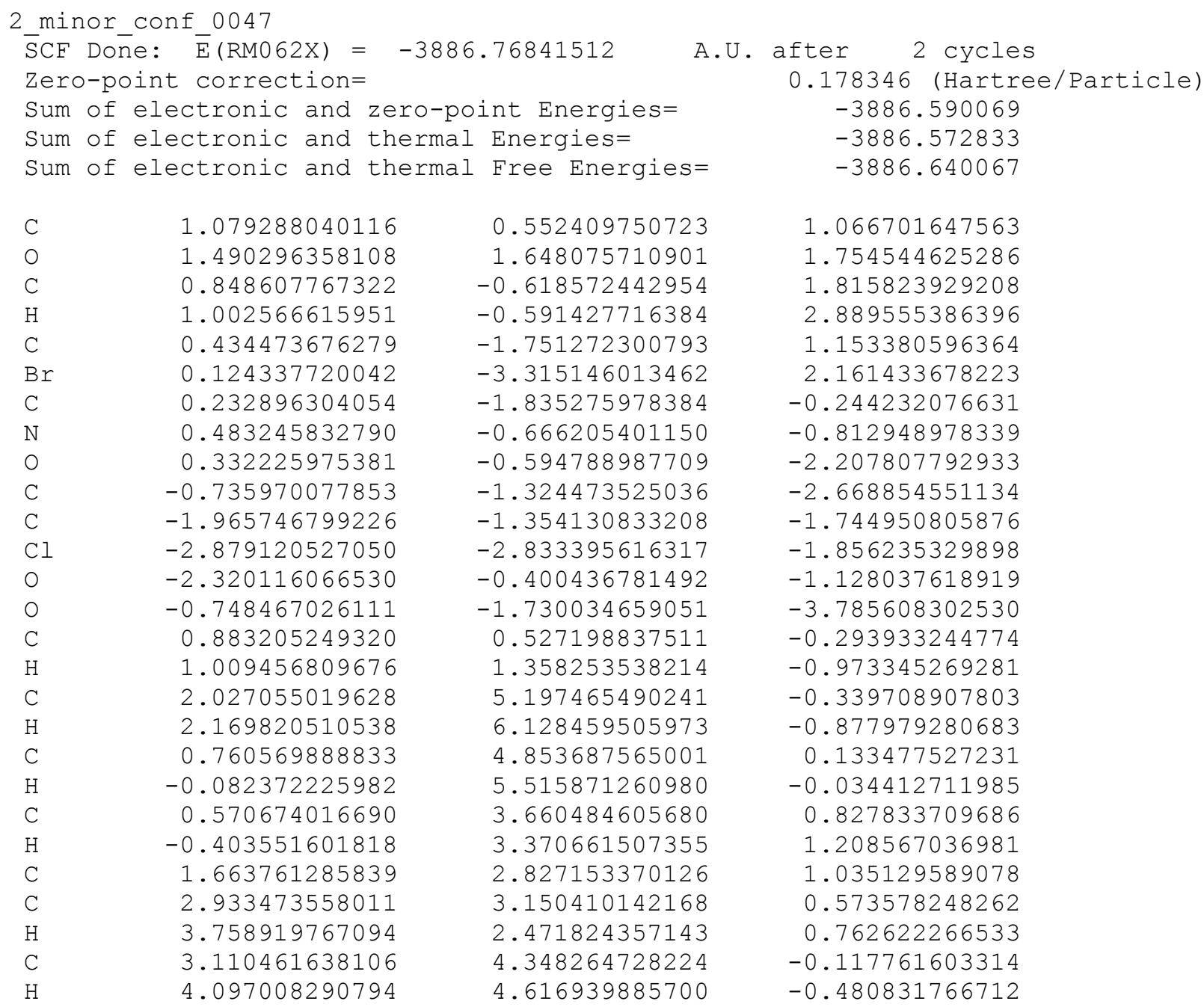




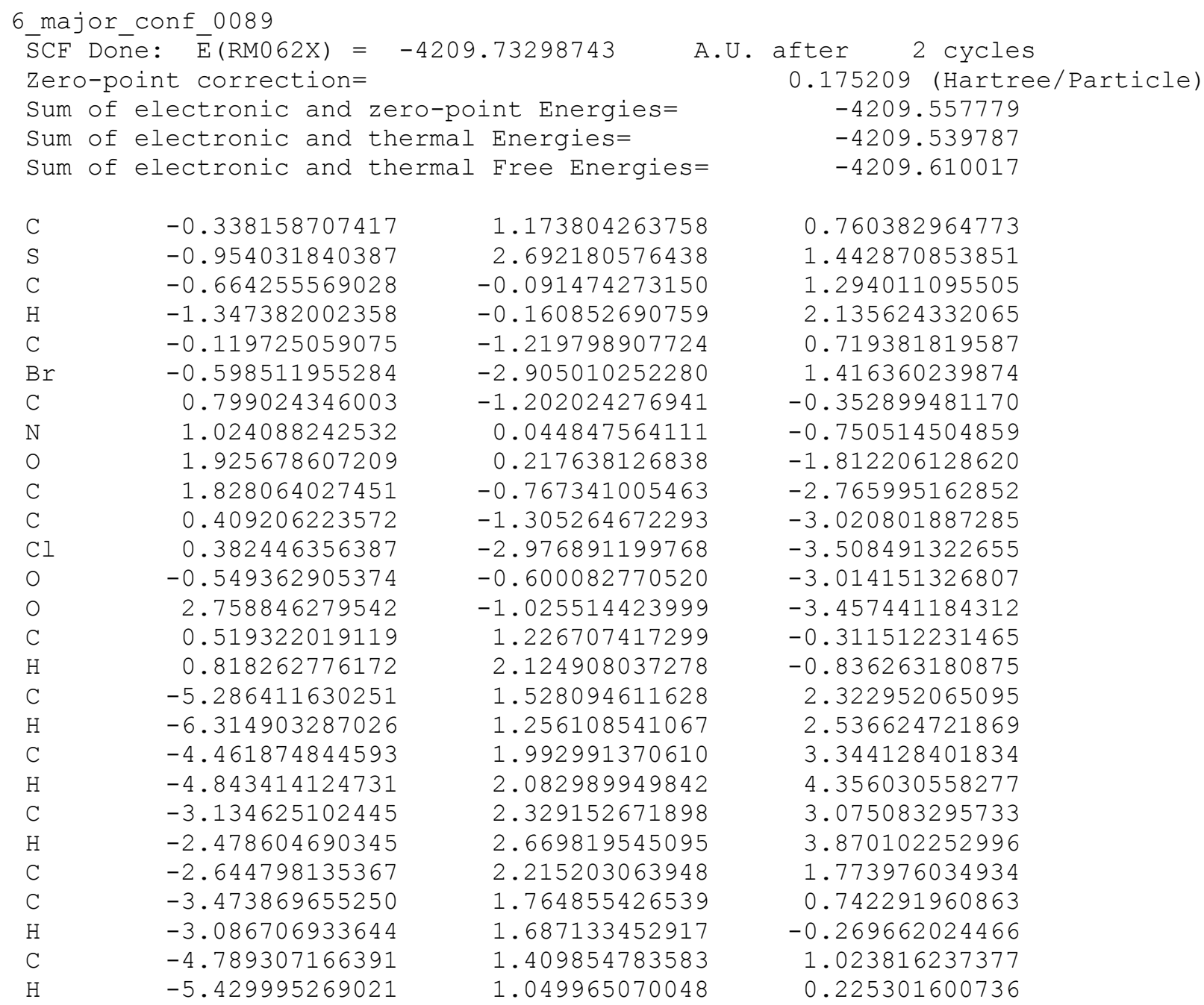




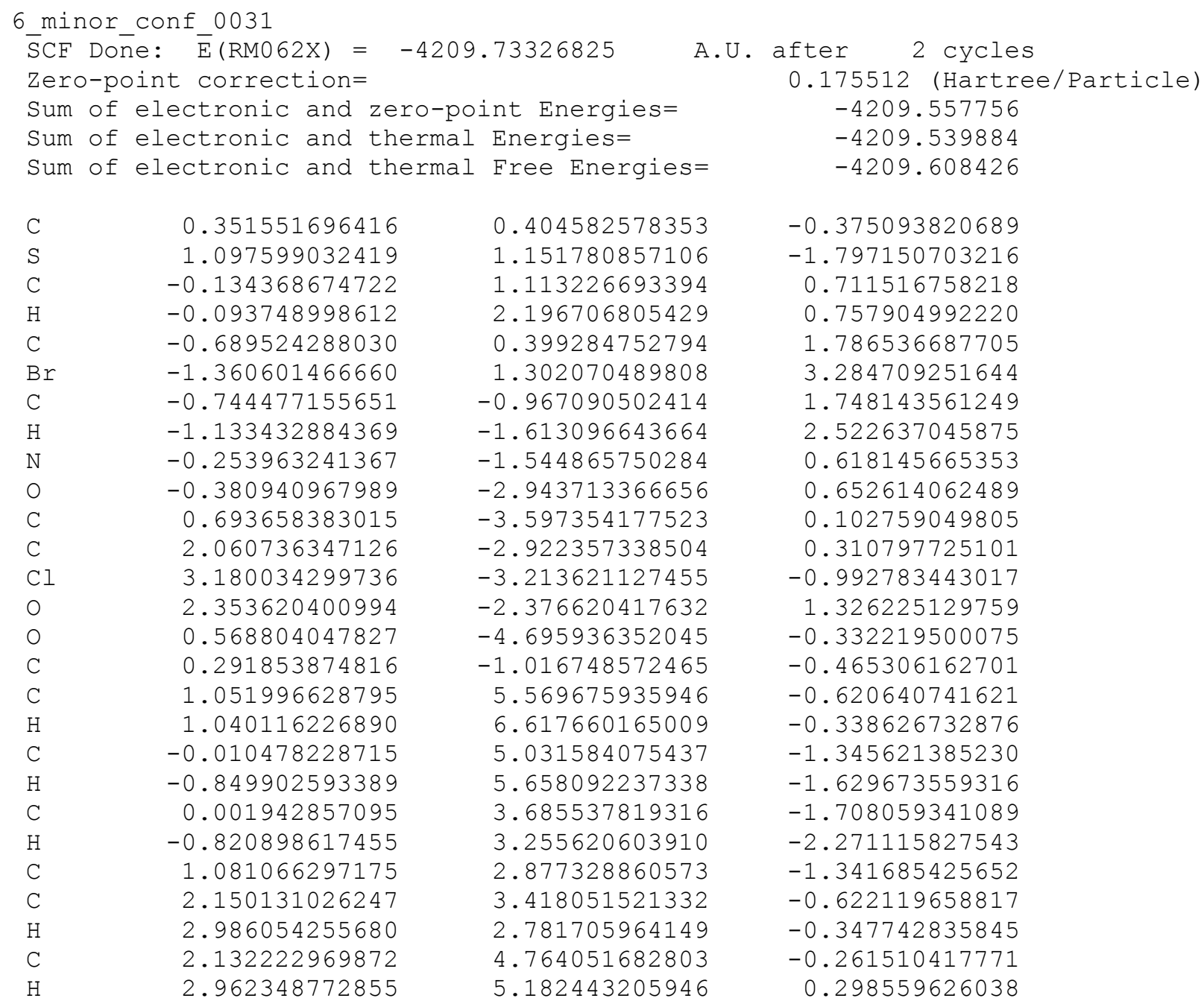




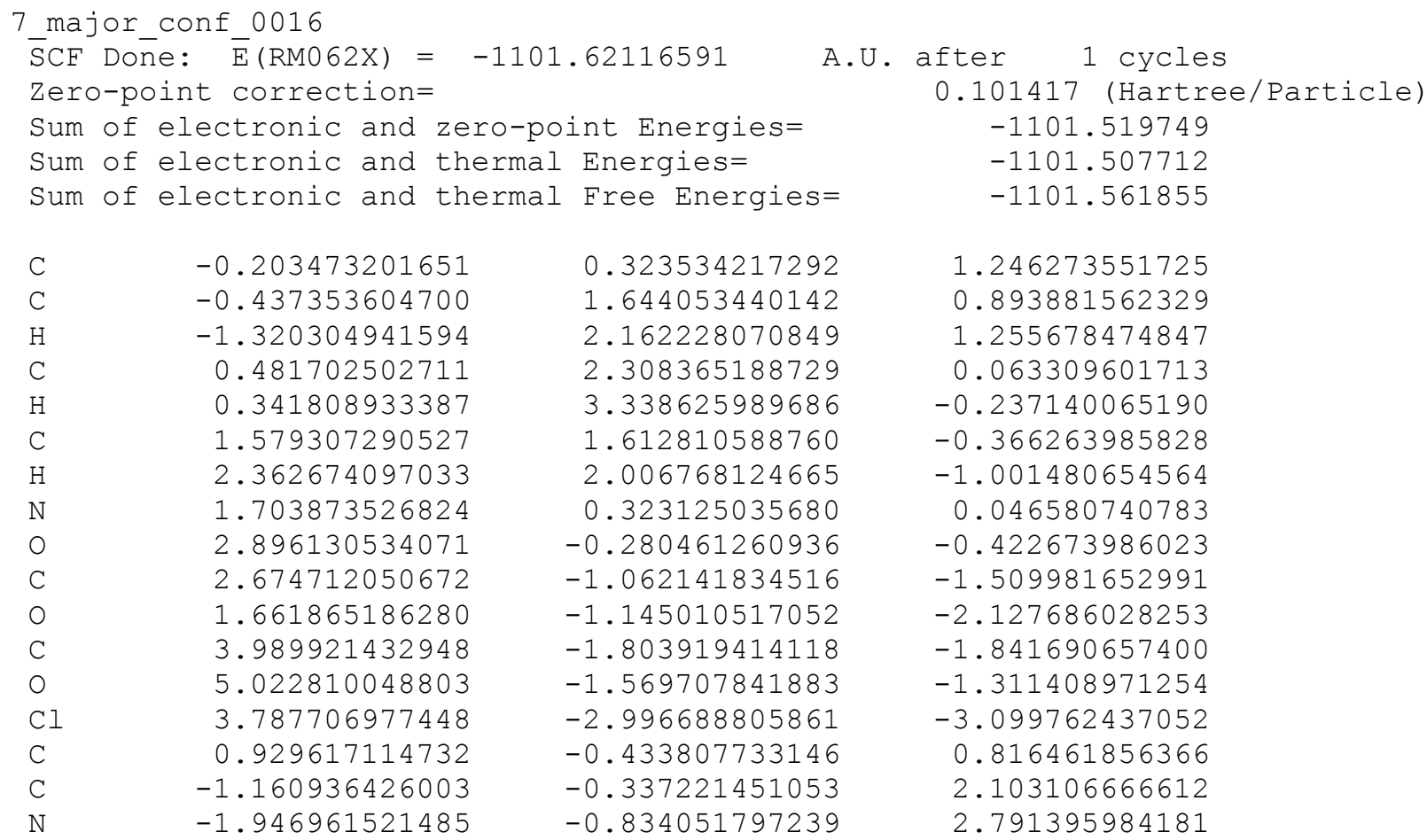




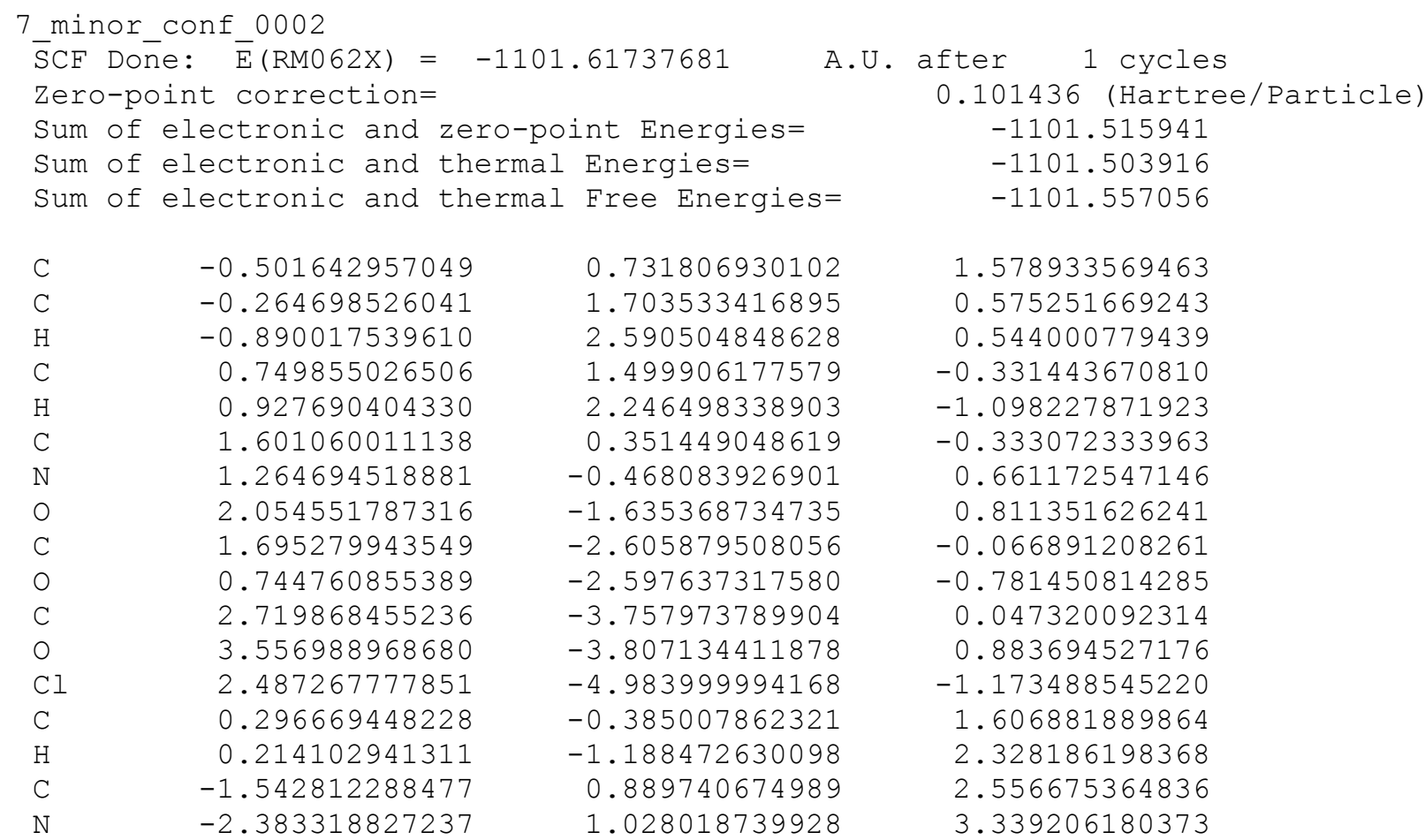




\begin{tabular}{|c|c|c|c|c|c|}
\hline \multicolumn{6}{|c|}{ 9_major_conf_0038 } \\
\hline $\bar{S} C F$ & Don & he: $\bar{E}(\mathrm{RM} 062$ & -3679 & .81971588 & after 1 cycles \\
\hline Zerc & & bint correct & Eion= & & 0.085089 (Hartree/Particle \\
\hline Sum & of & electronic & and zero-po & int Energies= & -3679.734627 \\
\hline Sum & of & electronic & and thermal & Energies= & -3679.722307 \\
\hline Sum & of & electronic & and thermal & - Free Energies= & -3679.777251 \\
\hline $\mathrm{C}$ & & -0.890268 & 3752975 & -0.368598726424 & 0.784082140670 \\
\hline $\mathrm{F}$ & & -1.992681 & 1624439 & -1.034552893827 & 1.154603901451 \\
\hline $\mathrm{C}$ & & -0.707534 & 4187730 & 0.888121478738 & 1.310243122568 \\
\hline $\mathrm{H}$ & & -1.427442 & 2962337 & 1.322904688487 & 1.995406632860 \\
\hline $\mathrm{C}$ & & 0.450016 & 6972463 & 1.590765280544 & 0.928638780124 \\
\hline $\mathrm{Br}$ & & 0.793437 & 7120591 & 3.306382010950 & 1.586834675297 \\
\hline $\mathrm{C}$ & & 1.329356 & 6985755 & 1.004226791876 & 0.060881607477 \\
\hline $\mathrm{H}$ & & 2.250077 & 7547088 & 1.446892974076 & -0.293582105059 \\
\hline $\mathrm{N}$ & & 1.007069 & 9266473 & -0.246826031917 & -0.373140066666 \\
\hline 0 & & 1.997861 & 1668683 & -0.779700178388 & -1.236021812476 \\
\hline $\mathrm{C}$ & & 1.663643 & 3189681 & -0.628511317883 & -2.542318868546 \\
\hline 0 & & 0.743029 & 9769643 & -0.009781304468 & -2.971096712537 \\
\hline $\mathrm{C}$ & & 2.716774 & 4498042 & -1.374142137491 & -3.394267256799 \\
\hline 0 & & 3.689187 & 7743297 & -1.874894537608 & -2.940222371954 \\
\hline $\mathrm{Cl}$ & & 2.325508 & 3719145 & -1.371428000052 & -5.095974778772 \\
\hline $\mathrm{C}$ & & -0.026535 & 5953382 & -1.028658096612 & -0.115166887640 \\
\hline
\end{tabular}




\begin{tabular}{|c|c|c|c|c|c|}
\hline \multicolumn{6}{|c|}{ 9_minor_conf_0013 } \\
\hline $\bar{S} C F$ & Dor & e: $\bar{E}(\mathrm{RM} 06$ & $2 \mathrm{X})=$ & .81398291 & $\begin{array}{l}\text { after } 1 \text { cycles } \\
0.084729 \text { (Hartree/Particle) }\end{array}$ \\
\hline Zer & & bint correc & tion= & & 0.084729 (Hartree/Particle) \\
\hline Sum & of & electronic & and zero-po & int Energies= & -3679.729253 \\
\hline Sum & of & electronic & and thermal & Energies= & -3679.716878 \\
\hline Sum & of & electronic & and thermal & Free Energies= & -3679.771874 \\
\hline $\mathrm{C}$ & & -0.30721 & 4193937 & 0.443240797498 & 1.421653222392 \\
\hline $\mathrm{F}$ & & -1.22339 & 5236046 & 0.259005781362 & 2.376481594208 \\
\hline $\mathrm{C}$ & & -0.18031 & 7212200 & 1.696816792338 & 0.808645704584 \\
\hline $\mathrm{H}$ & & -0.83074 & 6814356 & 2.509955953190 & 1.113934287127 \\
\hline $\mathrm{C}$ & & 0.78451 & 1158863 & 1.834461004290 & -0.166724298877 \\
\hline $\mathrm{Br}$ & & 0.96750 & 3391835 & 3.513040295326 & -1.001727988255 \\
\hline C & & 1.65274 & 7792805 & 0.797495451192 & -0.596373881233 \\
\hline $\mathrm{N}$ & & 1.40382 & 7566543 & -0.310552577363 & 0.078047581235 \\
\hline 0 & & 2.22548 & 0648685 & -1.426469536076 & -0.217964662698 \\
\hline C & & 1.80606 & 7761934 & -2.081722765803 & -1.329902776774 \\
\hline 0 & & 0.80202 & 7890728 & -1.881332298788 & -1.934668804977 \\
\hline $\mathrm{C}$ & & 2.84839 & 5328676 & -3.174633232511 & -1.660224583828 \\
\hline O & & 3.75138 & 2114630 & -3.452958876090 & -0.946123115896 \\
\hline $\mathrm{Cl}$ & & 2.52892 & 0863315 & -3.968252945663 & -3.182161874813 \\
\hline 0 & & 0.49685 & 5835706 & -0.596450275307 & 1.055619365868 \\
\hline $\mathrm{H}$ & & 0.46835 & 3102818 & -1.589543567593 & 1.482090231936 \\
\hline
\end{tabular}


11 major conf 0013

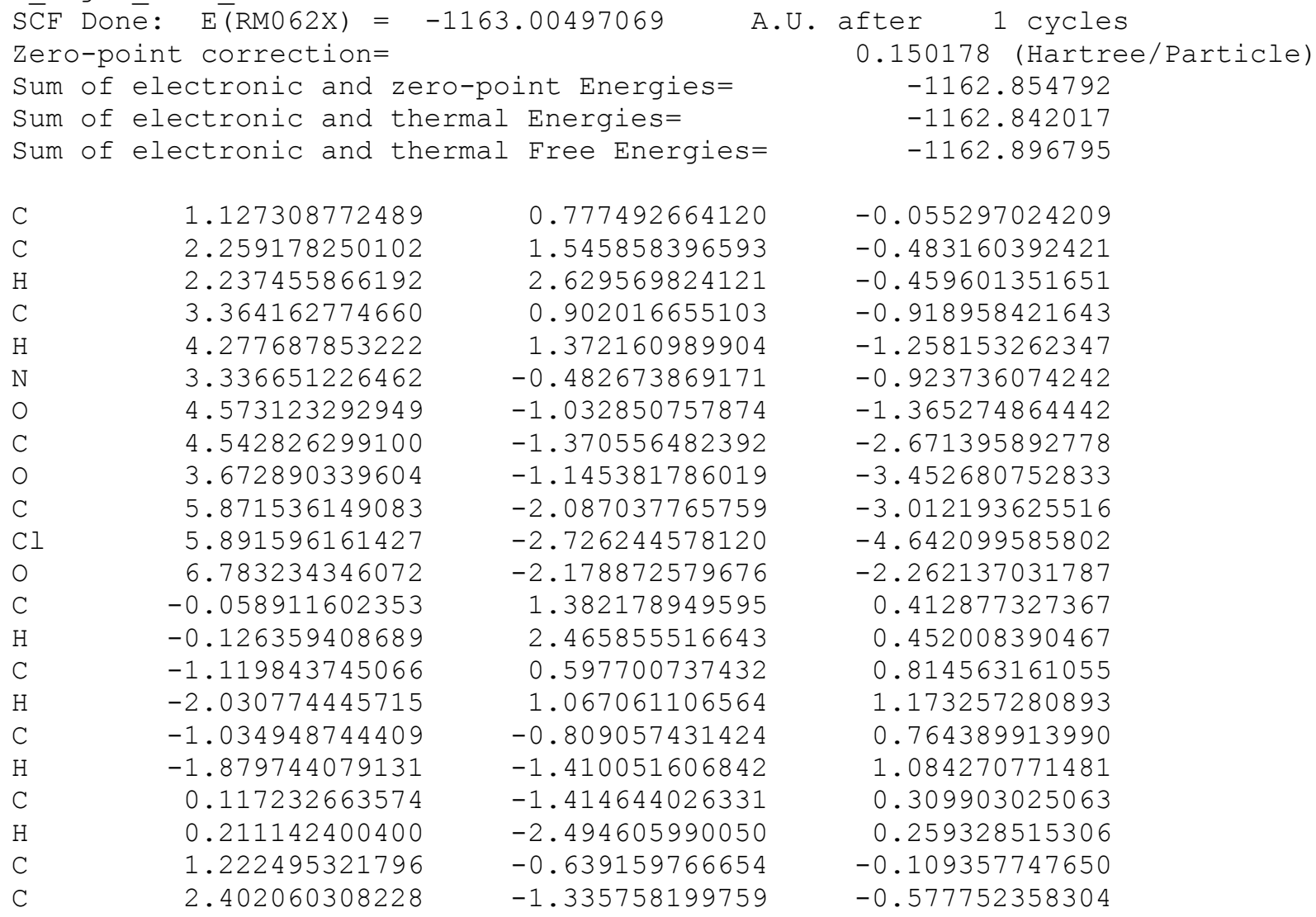


11 minor conf 0031

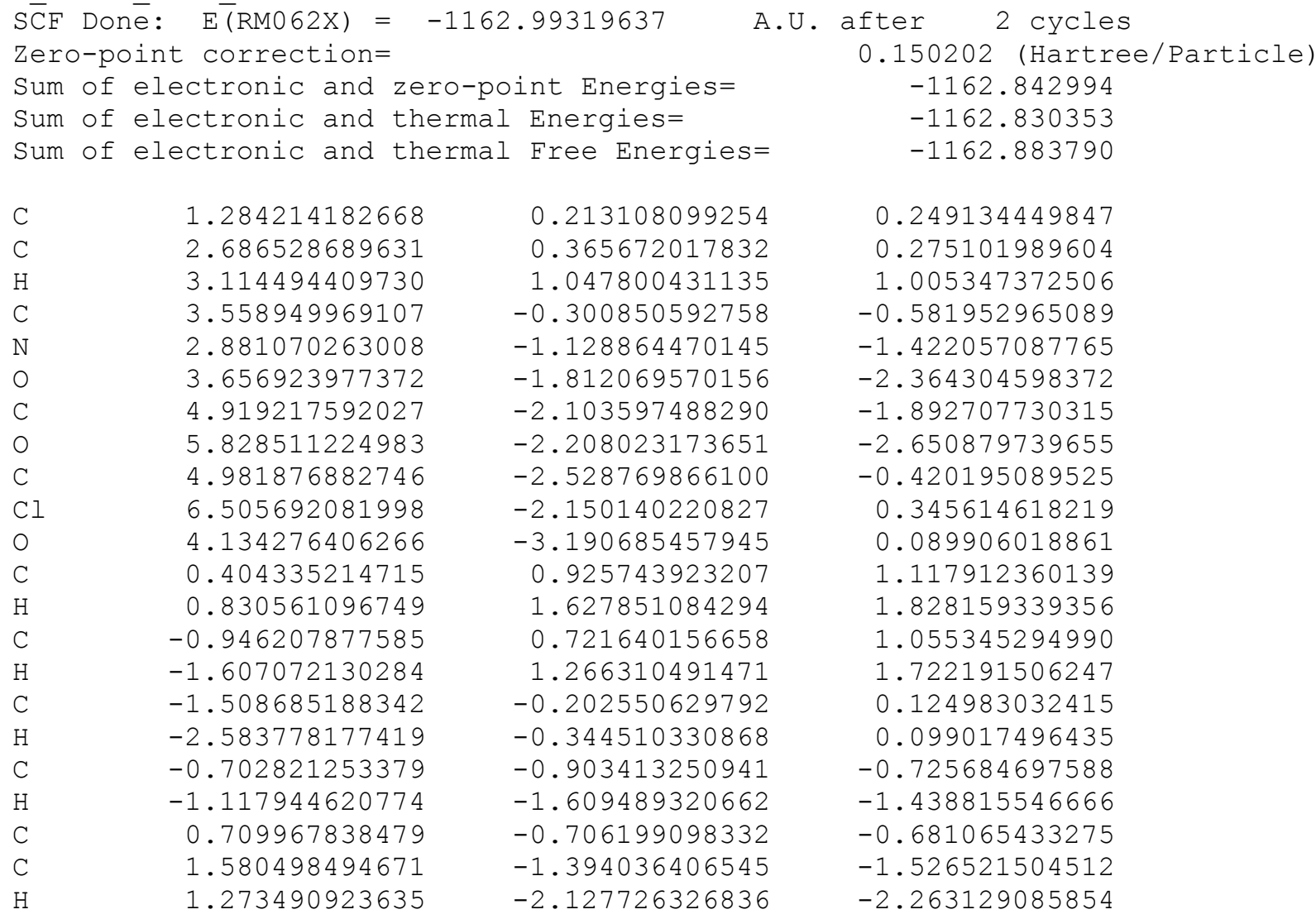


13 major conf 0098

$\begin{array}{lc}\mathrm{SC} F \text { Done }: \text { E (RM062X) }=-2000.02948085 \quad \text { A.U. after 2 cycles } \\ \text { Zero-point correction= } & 0.286887 \text { (Hartree/Particle) } \\ \text { Sum of electronic and zero-point Energies= } & -1999.742594 \\ \text { Sum of electronic and thermal Energies= } & -1999.718961 \\ \text { Sum of electronic and thermal Free Energies= } & -1999.800669\end{array}$

O

C

$\mathrm{H}$

C

S

C

$\mathrm{H}$

$\mathrm{N}$

O

C

C

$\mathrm{Cl}$

O

O

$\mathrm{H}$

C

$\mathrm{H}$

C

$\mathrm{H}$

C

$\mathrm{H}$

C

$\mathrm{H}$

C

$\mathrm{H}$

C

$\mathrm{H}$

C

$\mathrm{H}$

C

C

C

$\mathrm{H}$

$\mathrm{H}$

$\mathrm{H}$

$\mathrm{N}$
0.037090008256

0.445170388256

$-1.324987393919$

$-2.007717634606$

$-1.814694702065$

$-3.543138694022$

$-0.916231989024$

$-1.165895600362$

0.397718853879

1.232800368946

2.319080654834

2.083954722020

3.478395393431

1.074673082290

3.303798638814

0.992919957026

$-5.364454840788$

$-5.809525098784$

$-5.898322428537$

$-6.758866578177$

$-5.318668328835$

$-5.715222724335$

$-4.214196025791$

$-3.683967758834$

$-2.828664694194$

$-4.253774021824$

$-3.835608570759$

4.199954379427

5.177482426857

3.952543222069

4.731681808904

2.682178867837

2.413629951151

1.743575992892

2.064483987014

1.045634662555

0.847562917944

1.429305819228

0.094756155869

3.285821458847
$-0.447978683102$

$-1.740234471981$

$-0.224399296024$

$-1.051357451846$

1.083533016388

1.476518754576

2.100124089868

3.136120049550

1.755745489413

2.876709109982

2.629788807284

1.638189904244

0.677529267970

1.631635283540

3.287496315806

0.579337857847

$-1.751177134974$

$-2.511547488843$

$-1.490903420336$

$-2.049098863145$

$-0.524138608455$

$-0.335009729575$

0.196925789519

$-0.050176675395$

0.519499405534

$-1.032901951756$

$-1.229538058478$

$-2.873668237120$

$-3.196355318463$

$-2.719254133208$

$-2.913963467931$

$-2.309827524106$

$-2.167021459565$

$-2.074016968597$

$-2.254180318650$

$-2.003694529103$

$-0.931111974695$

$-2.383971989465$

$-2.489575765734$

$-2.653471809381$
$-1.119501575222$

$-1.282422622716$

$-1.201495570572$

$-1.375175476095$

$-1.029030327724$

$-1.121701070291$

$-0.803695253293$

$-0.618656733920$

$-0.800568848100$

$-0.634319177847$

0.165354824504

1.316570832371

1.707679890038

1.950022063797

0.063243928634

$-0.927924532083$

1. 558161279616

2. 191473137443

0.298858644082

$-0.055610782459$

$-0.523163333410$

$-1.515940635826$

$-0.068950077194$

1.200850467024

1. 553789837565

2. 004293020802

2. 986340074113

$-0.159305948242$

0.189601575800

$-1.520211999399$

$-2.248616937344$

$-1.914466244627$

$-2.956101228432$

$-0.926378818691$

0.424810757843

1.500297853778

1.614663967320

2. 447211080474

1.260693551262

0.789329298395 
13 minor conf 0091

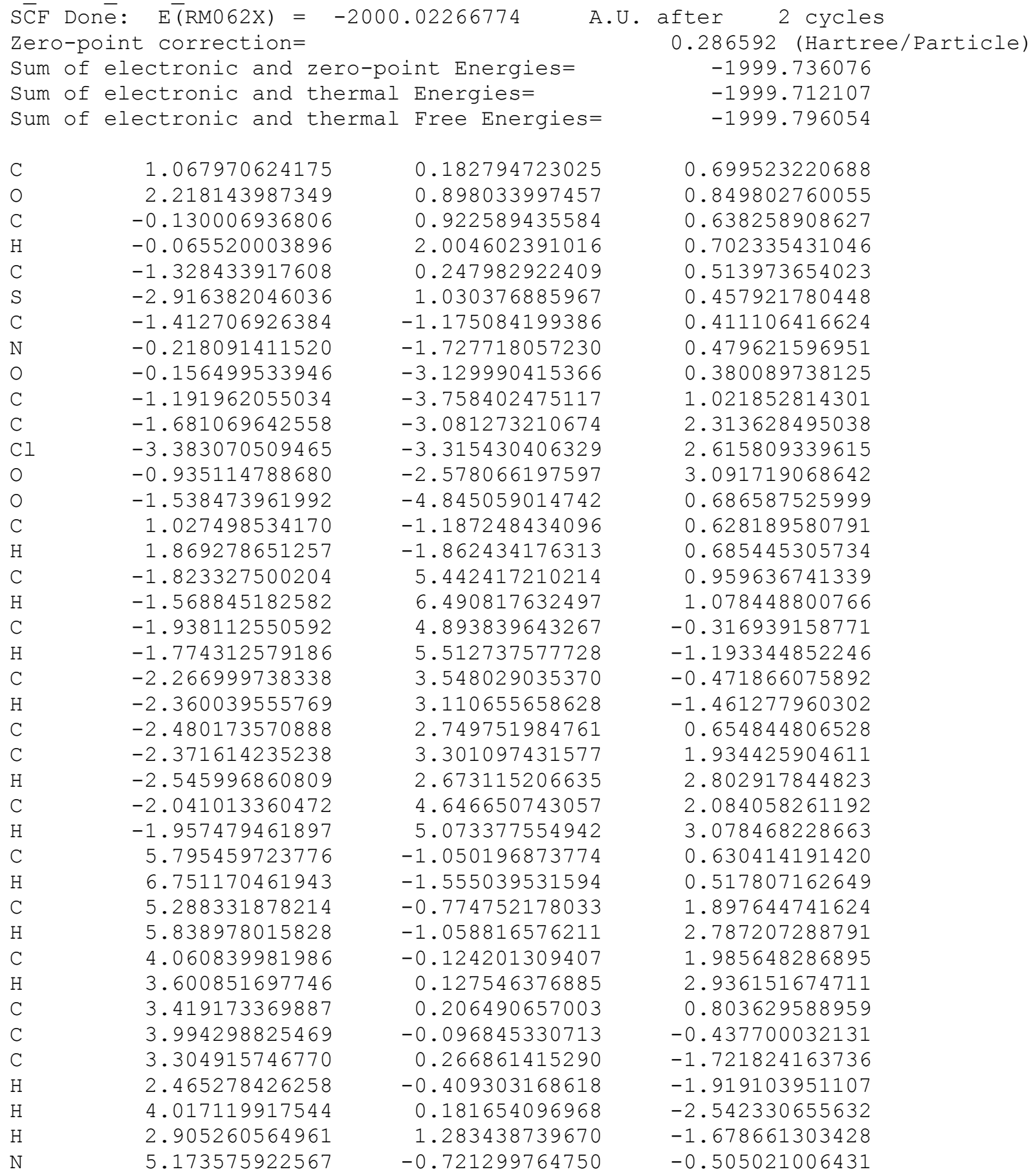


15 major conf 0006

\begin{tabular}{|c|c|c|c|}
\hline \multicolumn{4}{|c|}{-3847.71380723} \\
\hline Zero- & -point correction= & & 0.165002 (Hartree/Particle) \\
\hline um & of electronic and zero-poi & int Energies= & -3847.548806 \\
\hline Sum & of electronic and thermal & Energies= & -3847.531409 \\
\hline um & of electronic and thermal & Free Energies= & -3847.599543 \\
\hline C & -0.614109638162 & 0.005722814653 & 0.548403831152 \\
\hline & -0.516193934938 & 1.352143750006 & 0.146056729717 \\
\hline $\mathrm{H}$ & -1.265115651151 & 2.065784135662 & 0.472210336737 \\
\hline & 0.539278862541 & 1.719132550619 & -0.661677353382 \\
\hline $\mathrm{Br}$ & 0.656822860647 & 3.525985893050 & -1.203818573358 \\
\hline $\mathrm{C}$ & 1.553047264251 & 0.843001510799 & -1.118154928401 \\
\hline $\mathrm{N}$ & 1.325898584541 & -0.386057314989 & -0.662556917275 \\
\hline & 2.244789743728 & -1.382305144077 & -1.073084235300 \\
\hline $\mathrm{C}$ & 3.371889603326 & -1.370438761671 & -0.318419745859 \\
\hline 0 & 3.560506733977 & -0.727689294648 & 0.664255353124 \\
\hline C & 4.371329885583 & -2.374829647330 & -0.936526038876 \\
\hline 0 & 4.095990792692 & -3.110455165421 & -1.822780711538 \\
\hline $\mathrm{Cl}$ & 5.937716605313 & -2.320646046975 & -0.165678614631 \\
\hline $\mathrm{C}$ & 0.341640958400 & -0.881247571804 & 0.125432811209 \\
\hline $\mathrm{H}$ & 0.345819062133 & -1.935068599907 & 0.376057066667 \\
\hline $\mathrm{C}$ & -1.708854146509 & -0.525378933456 & 1.413303815957 \\
\hline 0 & -2.570741909666 & 0.426206520669 & 1.770785264721 \\
\hline $\mathrm{C}$ & -3.662168082355 & -0.001034071774 & 2.612828346121 \\
\hline $\mathrm{C}$ & -4.506859158018 & 1.218050621162 & 2.905175183721 \\
\hline $\mathrm{H}$ & -3.920270318675 & 1.980834460747 & 3.422506088340 \\
\hline $\mathrm{H}$ & -5.347970091415 & 0.936346327575 & 3.543361580463 \\
\hline $\mathrm{H}$ & -4.901958545619 & 1.646714391552 & 1.981217736979 \\
\hline n & -3.241493975618 & -0.444397916509 & 3.519412886535 \\
\hline & -4.219744871903 & -0.777654957858 & 2.082241205420 \\
\hline & -1791150633104 & -1.685219550073 & 1743248881760 \\
\hline
\end{tabular}




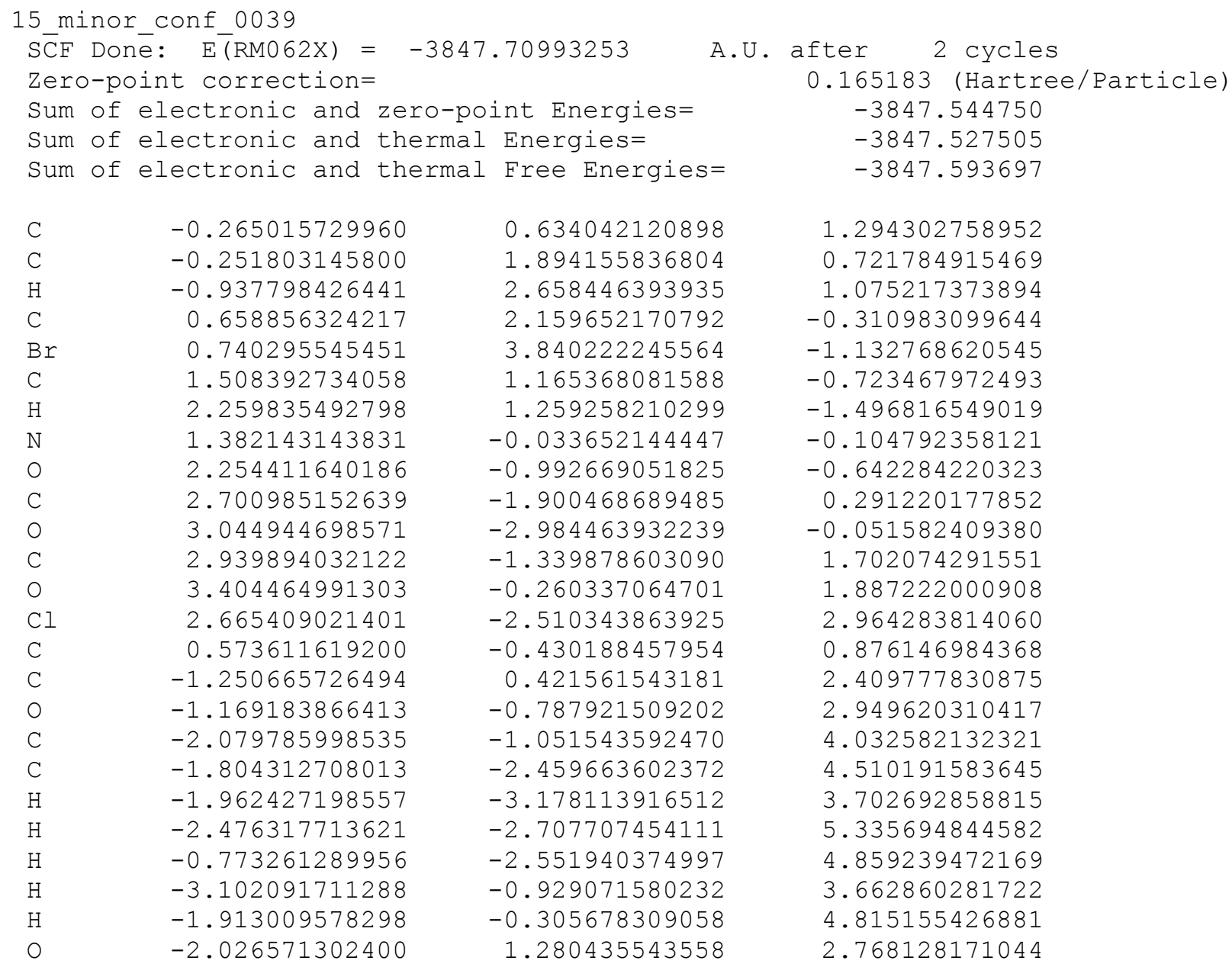


17 major conf 0056

$\begin{array}{lc}\mathrm{S} \bar{C} F \text { Done: } \mathrm{E}(\mathrm{RM} 6 \mathrm{XX})=-1944.69189800 \quad \text { A.U. after } 2 \text { cycles } \\ \text { Zero-point correction= } & 0.270907 \text { (Hartree/Particle) } \\ \text { Sum of electronic and zero-point Energies= } & -1944.420991 \\ \text { Sum of electronic and thermal Energies= } & -1944.398829 \\ \text { Sum of electronic and thermal Free Energies= } & -1944.477549\end{array}$

\begin{tabular}{lr}
$\mathrm{C}$ & 0.778276494726 \\
$\mathrm{O}$ & 1.788213249840 \\
$\mathrm{C}$ & 0.868570952853 \\
$\mathrm{H}$ & 1.730572821772 \\
$\mathrm{C}$ & -0.155089551593 \\
$\mathrm{~S}$ & 0.017308196235 \\
$\mathrm{C}$ & -1.210706503682 \\
$\mathrm{H}$ & -2.048008032489 \\
$\mathrm{~N}$ & -1.196707739810 \\
$\mathrm{O}$ & -2.372132269059 \\
$\mathrm{C}$ & -2.225403551341 \\
$\mathrm{C}$ & -0.849376660957 \\
$\mathrm{C}$ & -0.424685895756 \\
$\mathrm{O}$ & -0.240260335694 \\
$\mathrm{O}$ & -3.180803174889 \\
$\mathrm{C}$ & -0.327724544322 \\
$\mathrm{C}$ & -3.716386663506 \\
$\mathrm{H}$ & -4.603217964412 \\
$\mathrm{C}$ & -2.536763363091 \\
$\mathrm{H}$ & -2.502491634412 \\
$\mathrm{C}$ & -1.395928795204 \\
$\mathrm{H}$ & -0.471864005486 \\
$\mathrm{C}$ & -1.442495065603 \\
$\mathrm{C}$ & -2.621223002872 \\
$\mathrm{H}$ & -2.641881002603 \\
$\mathrm{C}$ & -3.758184913166 \\
$\mathrm{H}$ & -4.674903677236 \\
$\mathrm{C}$ & 3.279950232117 \\
$\mathrm{H}$ & 3.685942946765 \\
$\mathrm{C}$ & 2.986217398474 \\
$\mathrm{H}$ & 3.155977033998 \\
$\mathrm{C}$ & 2.463163473531 \\
$\mathrm{H}$ & 2.230986474602 \\
$\mathrm{C}$ & 2.232878157126 \\
$\mathrm{C}$ & 2.518137892398 \\
$\mathrm{H}$ & 2.321222159464 \\
$\mathrm{C}$ & 3.045070034901 \\
$\mathrm{H}$ & 3.267750828381 \\
& \\
\hline
\end{tabular}

$-0.018011630410$

$-1.753972601738$

$-2.672494002960$

$-0.716237161755$

0.886068203299

1.543571121776

0.931304726243

2.095615116795

$-0.646665484852$

0.255071064550

1. 581123149067

0.060749238038

$-0.034332742598$

$-0.753834918318$

$-1.526601022420$

$-2.724074809047$

$-3.401246559066$

$-4.253167426865$

$-3.493355358346$

$-3.266559598529$

$-0.884505215831$

1.202368407862

0.990490858976

0.498824577846

$-0.259714443216$

0.768857383908

0.227806577309

1.743367997575

2. 453264899724

3.210468789735

2.179199726890

2.728982033054

$-3.606327927667$

$-4.533137634329$

$-3.488973093797$

$-4.325275001018$

$-2.303526238630$

$-2.208647594316$

0.128974770490

0.806627742776

$-0.963397344573$

$-1.030603217344$

$-1.677903449135$

$-1.589886398067$

$-3.053144308371$

$-0.570130007204$

$-2.135956914022$

$-1.948495450742$

4.057264392882

4.645815460336

4.298656138598

5.073847312040

3.545287103882

3. 724080465322

2.545331712232

2. 305009033091

1.527385942582

3. 063233147775

2. 876484741846

$-4.141125019494$

$-4.532555793053$

$-2.783491704367$

$-2.112760478378$

$-2.273752399712$

$-1.217122128336$

$-1.239438311597$

$-1.337932488326$

$-0.487606092984$

$-2.528531107544$

$-2.613929171982$

$-3.142528428086$

$-4.499060058615$

$-5.142714101283$

$-4.994356341690$

$-6.053239383690$ 
17 minor conf 0071

\begin{tabular}{|c|c|c|c|}
\hline $\begin{array}{l}\text { SCF } \\
\text { Zerc }\end{array}$ & 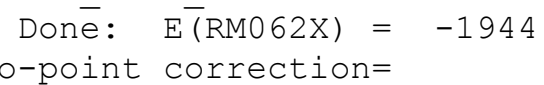 & .68725921 & $\begin{array}{l}\text { after } 2 \text { cycles } \\
0.270760 \text { (Hartree/Particle) }\end{array}$ \\
\hline Sum & of electronic and zero-po: & int Energies= & -1944.416499 \\
\hline Sum & of electronic and thermal & Energies= & -1944.394168 \\
\hline um & of electronic and thermal & - Free Energies= & -1944.474592 \\
\hline $\mathrm{C}$ & 1.670685459724 & 0.925691608825 & -0.283005041663 \\
\hline 0 & 2.997432610513 & 1.208823868854 & -0.387137991253 \\
\hline $\mathrm{C}$ & 0.899133692253 & 1.800401352908 & 0.509037599929 \\
\hline $\mathrm{H}$ & 1.401433275041 & 2.642966977615 & 0.974615720022 \\
\hline & -0.453559320070 & 1.561909546468 & 0.649740998955 \\
\hline S & -1.559225676826 & 2.585109896673 & 1.582765587270 \\
\hline $\mathrm{C}$ & -1.113765893749 & 0.448576784431 & 0.045305397215 \\
\hline $\mathrm{N}$ & -0.268676536576 & -0.280130147188 & -0.656683527403 \\
\hline & -0.792102053422 & -1.416202855174 & -1.302385152212 \\
\hline $\mathrm{C}$ & -2.043056752502 & -1.205475618384 & -1.819757689103 \\
\hline $\mathrm{C}$ & -2.303962565581 & 0.207489896757 & -2.369083640099 \\
\hline $\mathrm{Cl}$ & -3.963354737368 & 0.720958139150 & -2.202113653036 \\
\hline 0 & -1.482792849391 & 0.817443632185 & -2.976525333338 \\
\hline 0 & -2.786949712673 & -2.116819601340 & -1.993042293458 \\
\hline & 1.069884188578 & -0.148779607319 & -0.891510364237 \\
\hline $\mathrm{H}$ & 1.546468135357 & -0.876794140538 & -1.531659404253 \\
\hline $\mathrm{C}$ & 1.224215236113 & 5.906901907383 & 3.043341195092 \\
\hline $\mathrm{H}$ & 1.881707789843 & 6.697095310914 & 3.391697600485 \\
\hline $\mathrm{C}$ & 0.579785416834 & 6.028829455362 & 1.812667610261 \\
\hline $\mathrm{H}$ & 0.733265962301 & 6.912827922427 & 1.201997136557 \\
\hline r & -0.266940911407 & 5.016833098285 & 1.364099449825 \\
\hline $\mathrm{H}$ & -0.775369096658 & 5.102131178550 & 0.408465707272 \\
\hline $\mathrm{C}$ & -0.466214340120 & 3.877674352700 & 2.148686009753 \\
\hline $\mathrm{C}$ & 0.175088250238 & 3.757969871447 & 3.384215901731 \\
\hline $\mathrm{H}$ & 0.008526528943 & 2.871153370659 & 3.988119403016 \\
\hline $\mathrm{C}$ & 1.020466075751 & 4.773238396784 & 3.828930048547 \\
\hline $\mathrm{H}$ & 1.517455327478 & 4.678581694238 & 4.789133755316 \\
\hline $\mathrm{C}$ & 5.317656862201 & -0.966690520130 & -3.062895566380 \\
\hline $\mathrm{H}$ & 5.933004854613 & -1.527888947553 & -3.758124954254 \\
\hline C & 5.392624875246 & -1.229919430196 & -1.696268128963 \\
\hline $\mathrm{H}$ & 6.066545689553 & -1.994892469154 & -1.324714285060 \\
\hline $\mathrm{C}$ & 4.602545012072 & -0.512500984671 & -0.798191053368 \\
\hline $\mathrm{H}$ & 4.640866119875 & -0.695422313828 & 0.270547682404 \\
\hline $\mathrm{C}$ & 3.749016345182 & 0.466364572353 & -1.290265391625 \\
\hline C & 3.663043193727 & 0.747881209747 & -2.650188166340 \\
\hline $\mathrm{H}$ & 2.986001332846 & 1.523106687913 & -2.995424116081 \\
\hline $\mathrm{C}$ & 4.453523050934 & 0.021041186194 & -3.537288179666 \\
\hline$H$ & 4.396895161126 & 0.230814716653 & -4.600402871858 \\
\hline
\end{tabular}




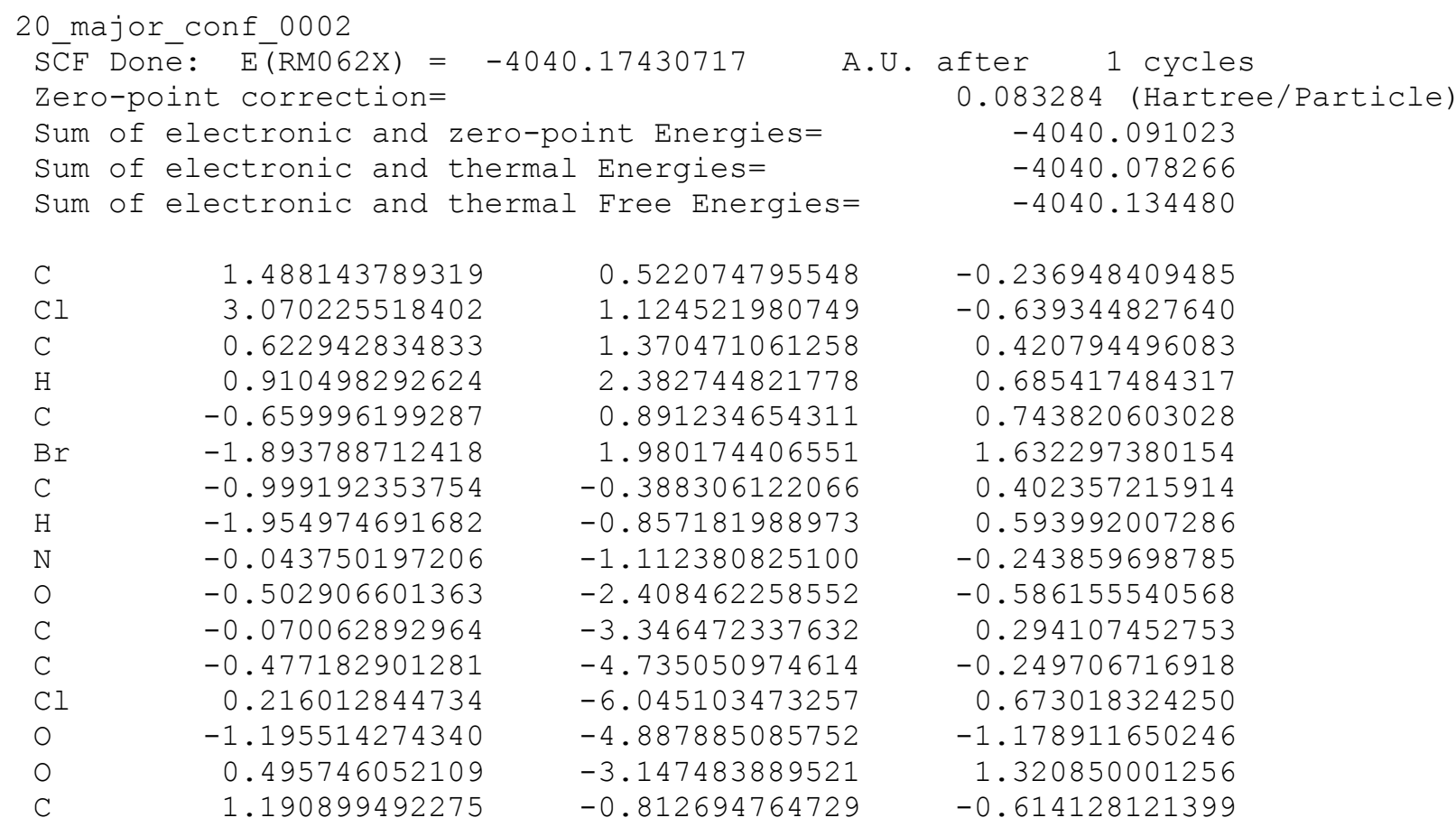




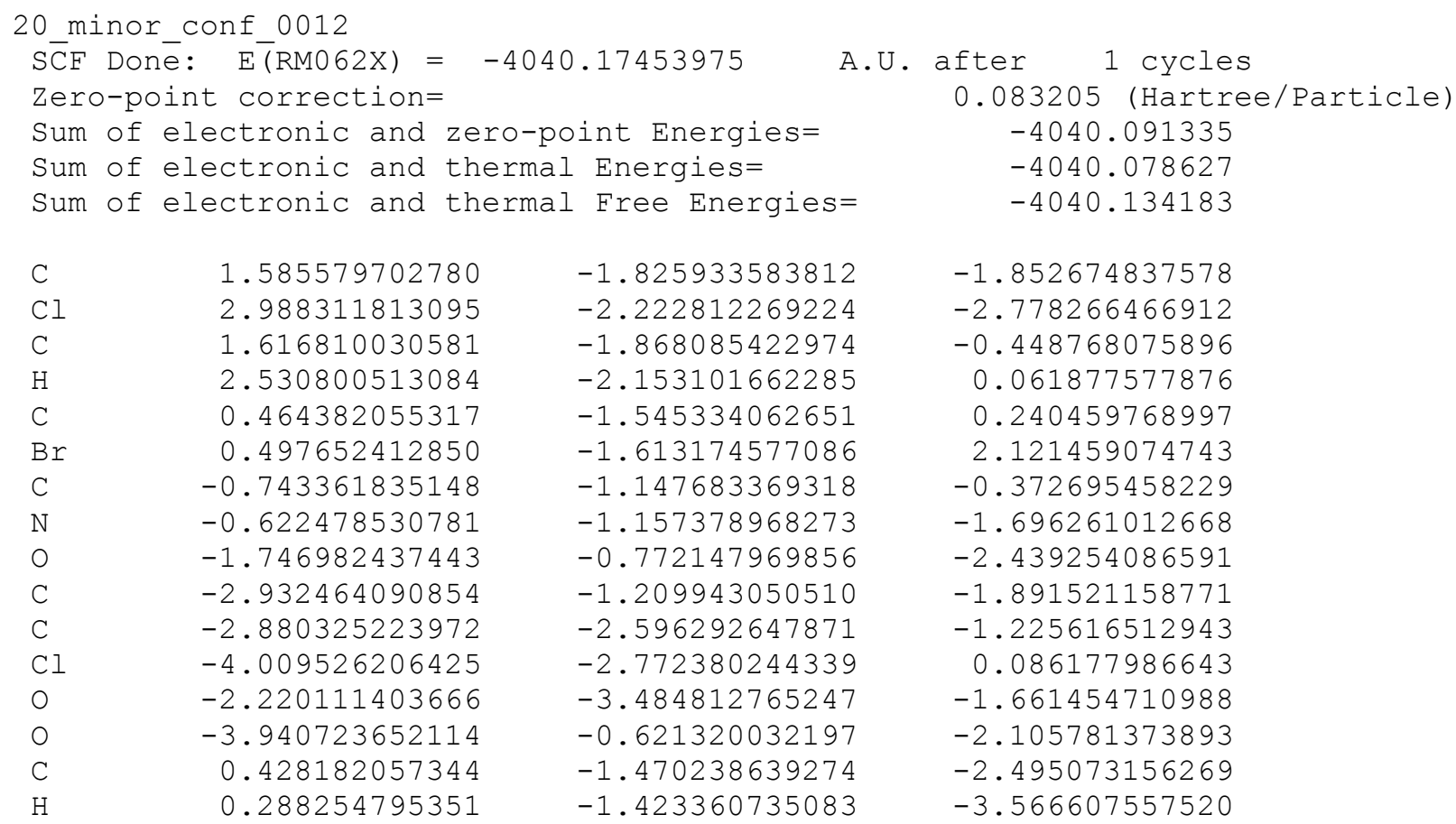




\section{S7. M06-2X/6-31+G(d,p) coordinates and energies of major and minor halogenated products for species $2,6,7,9,11,13,15,17$ and 20}

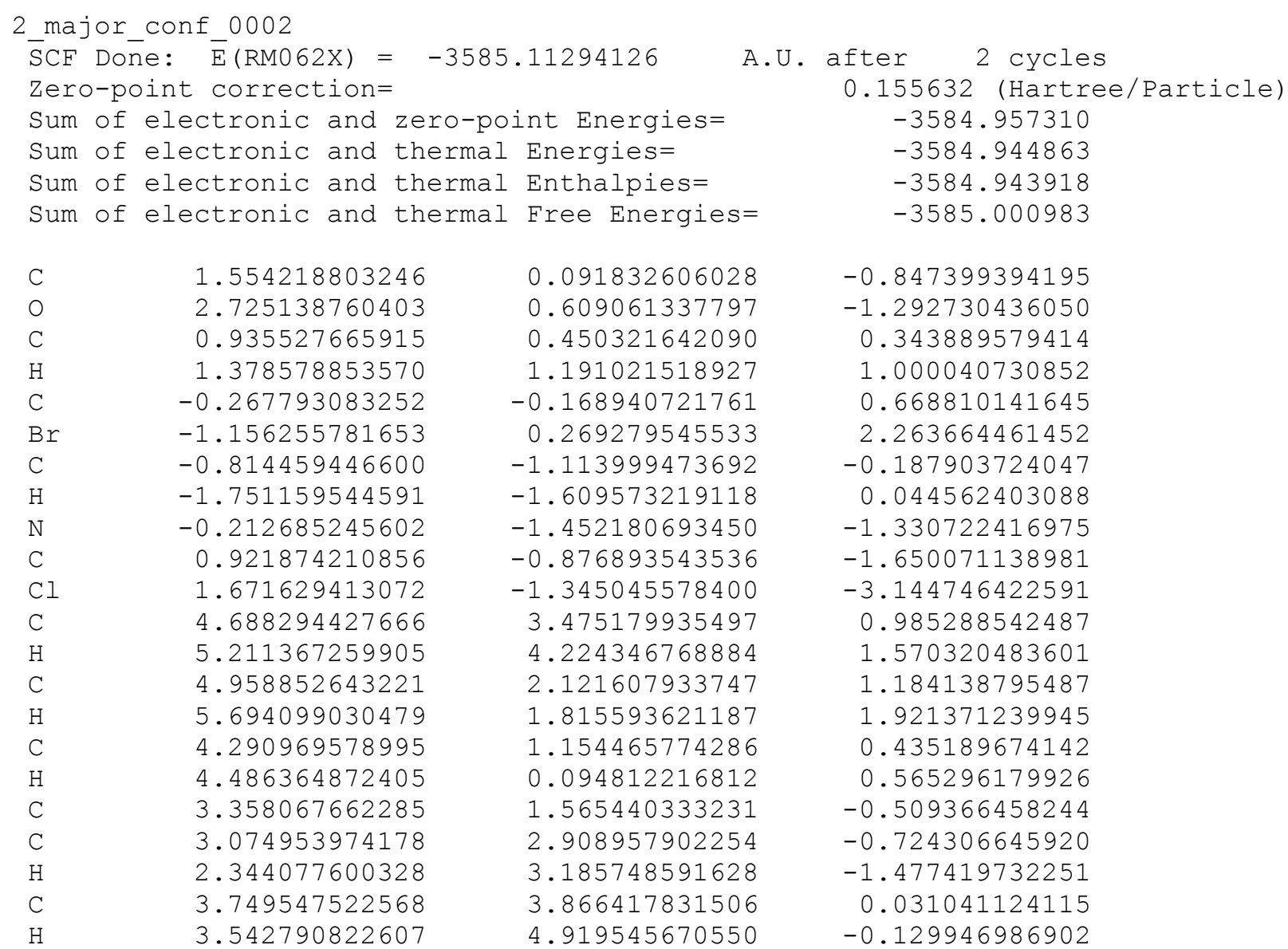




\begin{tabular}{|c|c|c|c|c|c|}
\hline \multicolumn{6}{|c|}{ _minor_conf_0001 } \\
\hline Zer & 0 & $\begin{array}{l}\text { ne: } \\
\text { oint }(\text { RMO } \\
\text { orrection })= \\
\end{array}$ & & & $\begin{array}{l}\text { atter } 2 \text { cycles } \\
0.155501 \text { (Hartree/Partic] }\end{array}$ \\
\hline Sum & of & electronic and $z$ & zero-po & int Energies= & -3584.956683 \\
\hline Sum & of & electronic and $t$ & thermal & Energies= & -3584.944321 \\
\hline Sum & of & electronic and $t$ & thermal & Enthalpies= & -3584.943377 \\
\hline Sum & of & electronic and $t$ & thermal & Free Energies= & -3585.000422 \\
\hline C & & 1.39661009530 & & 0.799681536591 & -0.167920019430 \\
\hline 0 & & 2.61708480568 & & 1.389276384704 & -0.322736592448 \\
\hline $\mathrm{C}$ & & 0.35107868297 & & 1.602265107745 & 0.277600887267 \\
\hline $\mathrm{H}$ & & 0.53406059784 & & 2.641104115977 & 0.528705140281 \\
\hline C & & -0.91449747263 & & 1.045700358132 & 0.388422303027 \\
\hline $\mathrm{Br}$ & & -2.33630194160 & & 2.105382729202 & 0.991668026824 \\
\hline C & & -1.07910469521 & & -0.304672951155 & 0.048927307807 \\
\hline $\mathrm{Cl}$ & & -2.63194920896 & & -1.072243097918 & 0.165684347440 \\
\hline $\mathrm{N}$ & & -0.08509725552 & & -1.056081039719 & -0.377273550382 \\
\hline C & & 1.13309248940 & & -0.531241565415 & -0.493464105234 \\
\hline $\mathrm{H}$ & & 1.92085914444 & & -1.187332698556 & -0.851143712877 \\
\hline $\mathrm{C}$ & & 6.11463869657 & & -0.831776346999 & 0.019406385622 \\
\hline $\mathrm{H}$ & & 7.03723507381 & & -1.395699325914 & 0.107305215975 \\
\hline $\mathrm{C}$ & & 5.89339359387 & & -0.020732149199 & -1.091842018640 \\
\hline $\mathrm{H}$ & & 6.64298560524 & & 0.049798194631 & -1.873492740627 \\
\hline C & & 4.70699111551 & & 0.701433559581 & -1.212504708990 \\
\hline $\mathrm{H}$ & & 4.50953257291 & & 1.338171110116 & -2.068259745884 \\
\hline $\mathrm{C}$ & & 3.75451509058 & & 0.605549893154 & -0.205808017664 \\
\hline C & & 3.96305451062 & & -0.188646974107 & 0.919514579686 \\
\hline $\mathrm{H}$ & & 3.20730699734 & & -0.234582880524 & 1.697832336015 \\
\hline C & & 5.14784558982 & & -0.912279959549 & 1.022217087423 \\
\hline $\mathrm{H}$ & & 5.31796591198 & & -1.535174000779 & 1.894561594810 \\
\hline
\end{tabular}




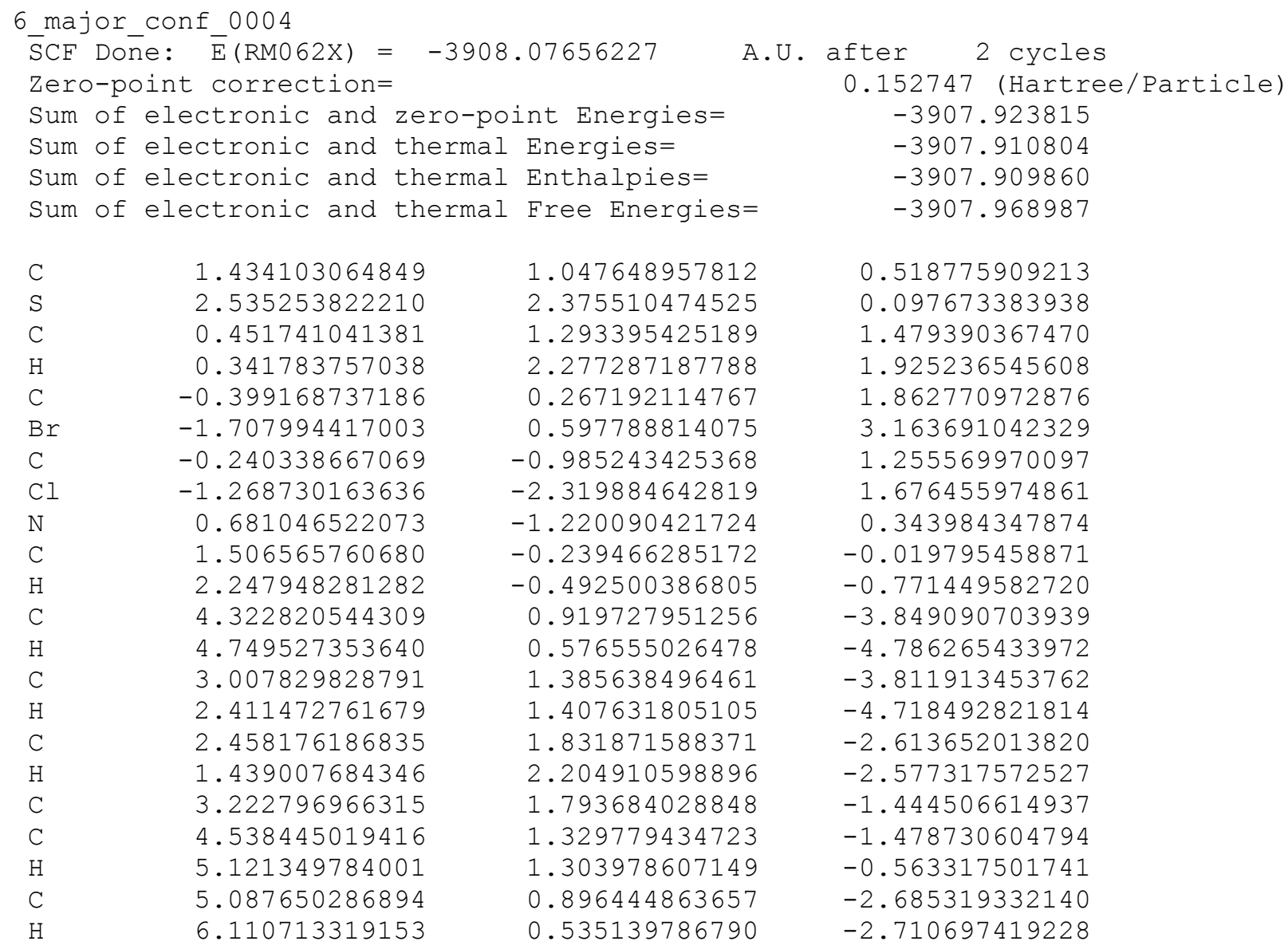




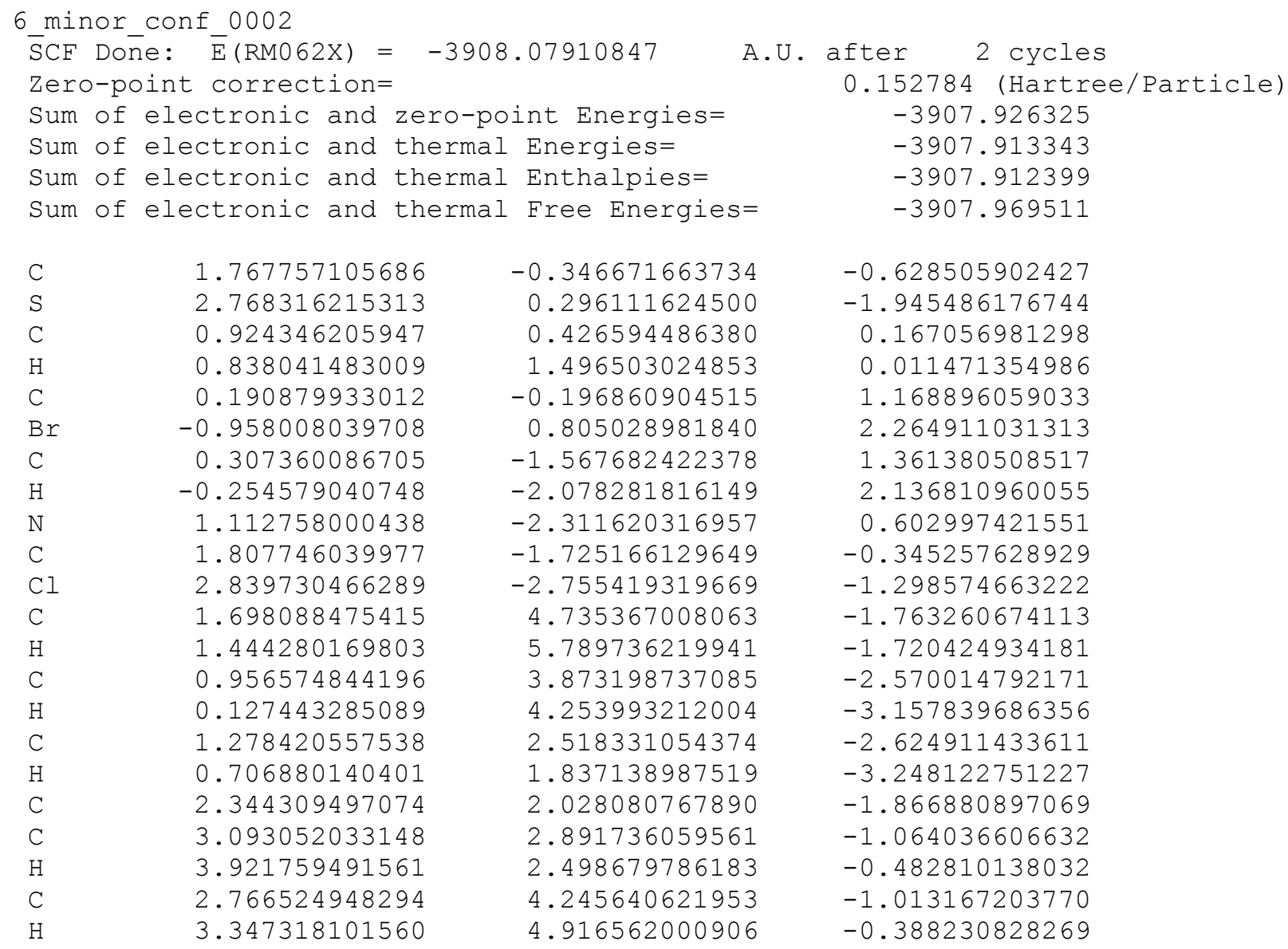




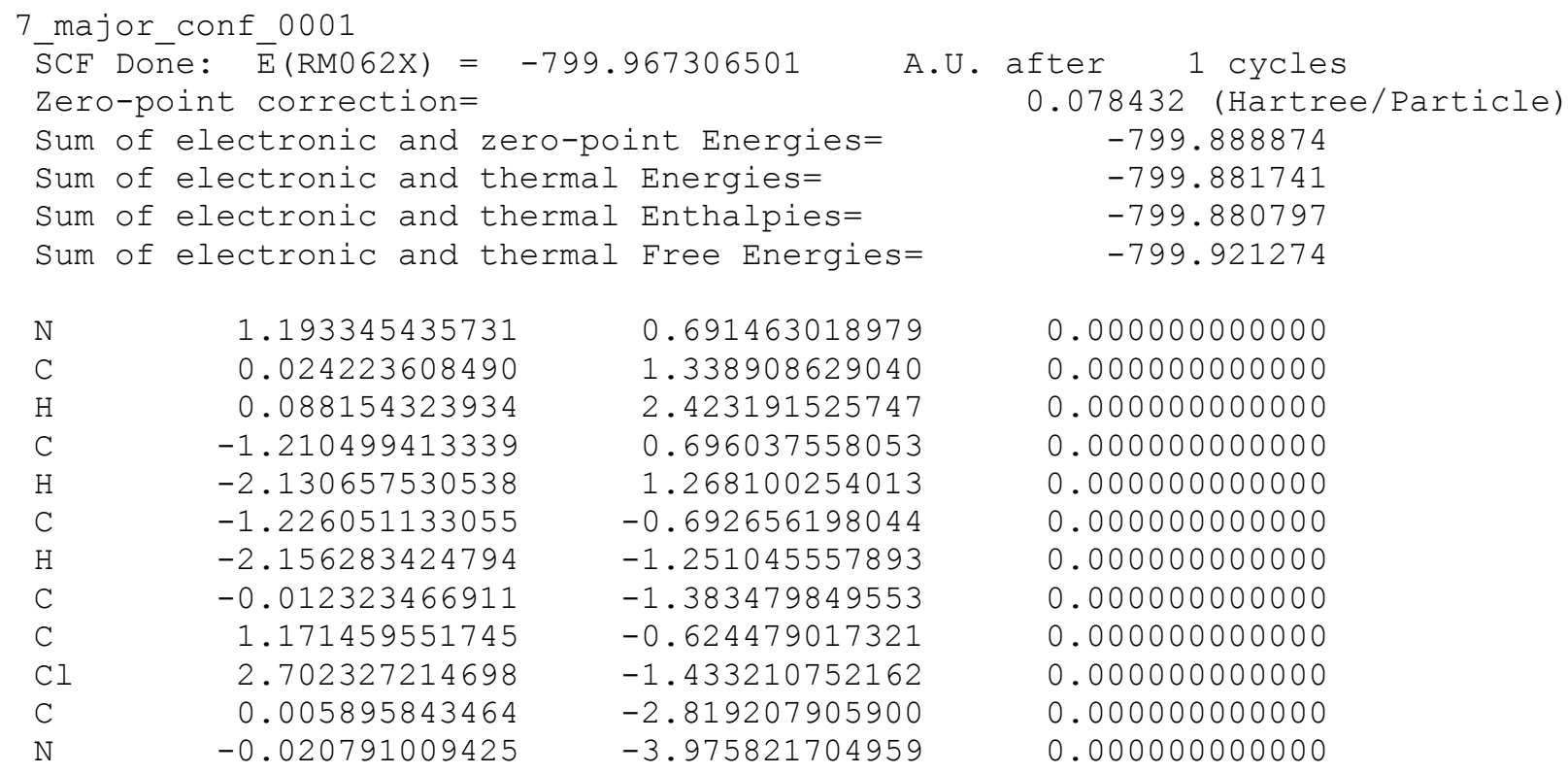




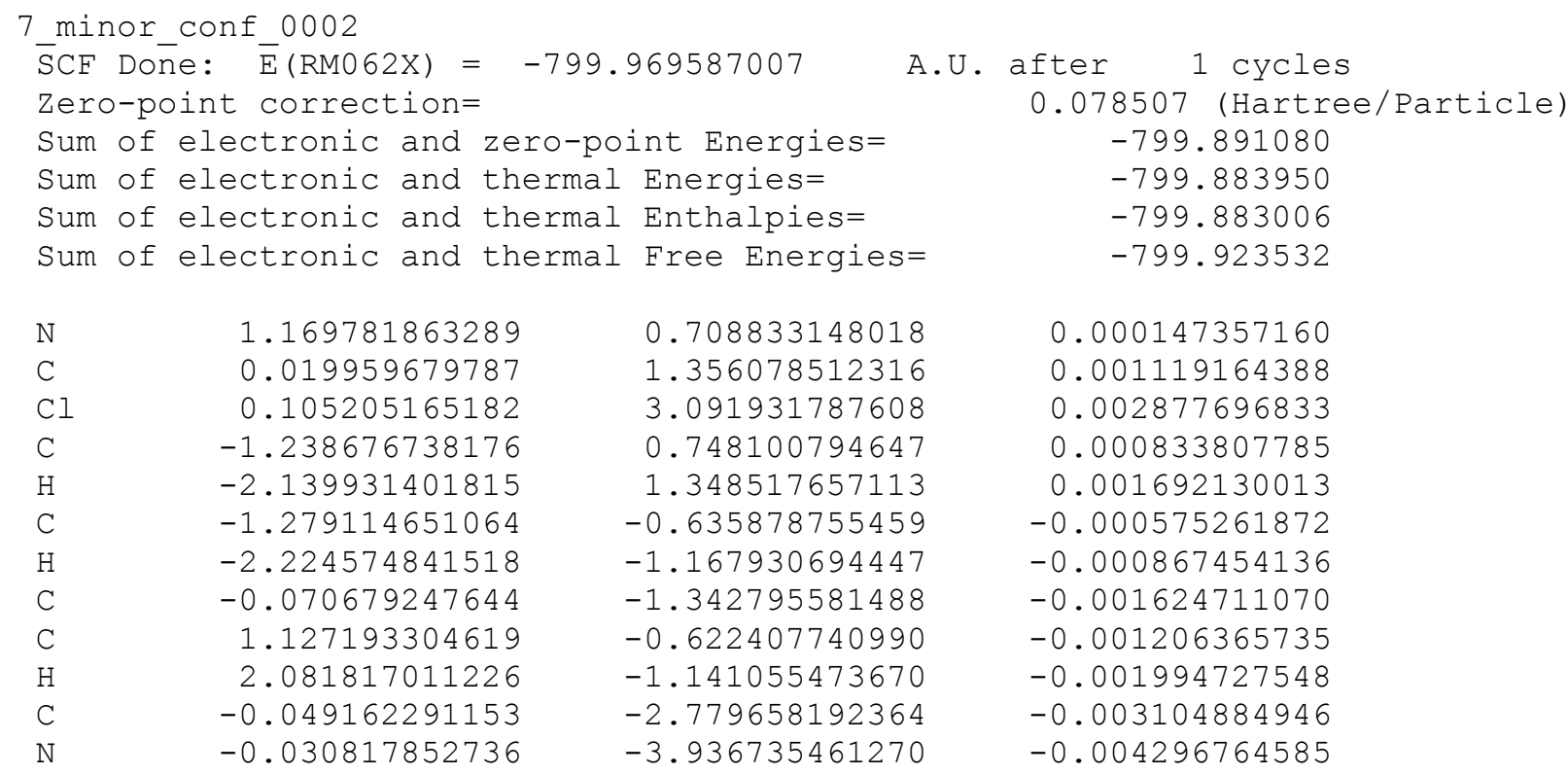




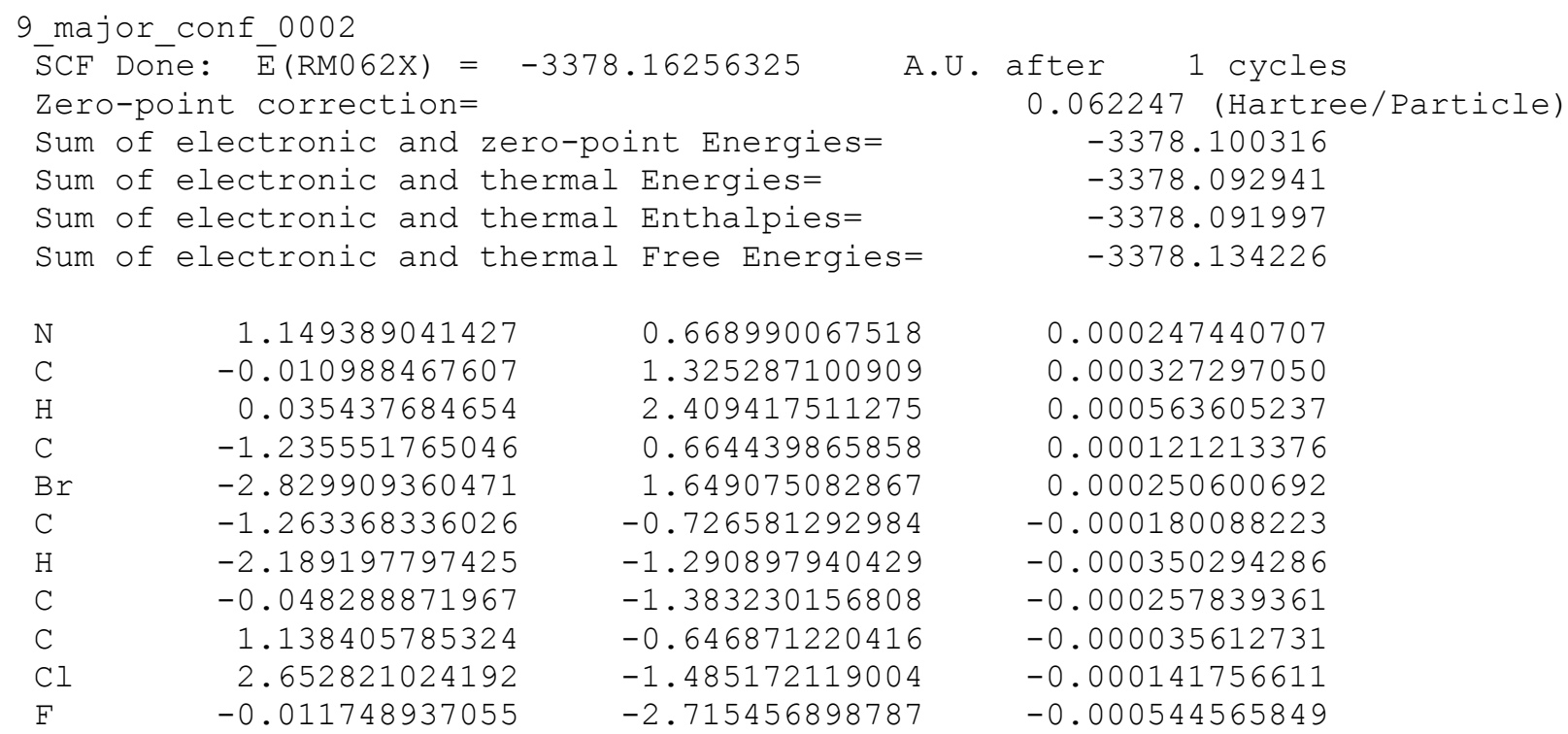




\begin{tabular}{|c|c|c|c|c|c|}
\hline \multicolumn{6}{|c|}{ _minor_conf_o002 } \\
\hline$\overline{\mathrm{S}} \mathrm{CF}$ & Don & ne: $\bar{E}(\operatorname{RM062X)}=$ & -3378 & .16250807 & after 2 cycles \\
\hline Zer & & oint correction= & & & 0.062123 (Hartree/Particle \\
\hline Sum & of & electronic and & zero-po & int Energies= & -3378.100385 \\
\hline Sum & of & electronic and $t$ & thermal & Energies= & -3378.093019 \\
\hline Sum & of & electronic and $t$ & thermal & Enthalpies= & -3378.092075 \\
\hline Sum & of & electronic and & thermal & - Free Energies= & -3378.134267 \\
\hline $\mathrm{N}$ & & 1.1301060503 & & 0.709942534691 & -0.002194170748 \\
\hline $\mathrm{C}$ & & $-0.0131417584 \varepsilon$ & & 1.365997075365 & -0.000809625362 \\
\hline $\mathrm{Cl}$ & & 0.09845003313 & & 3.097289021807 & 0.000007102426 \\
\hline $\mathrm{C}$ & & -1.2632223100 & & 0.728308125010 & -0.000015491660 \\
\hline $\mathrm{Br}$ & & -2.8707325122 & & 1.682099579091 & 0.001942157974 \\
\hline $\mathrm{C}$ & & -1.28820205413 & & -0.660884919127 & -0.000730080002 \\
\hline $\mathrm{H}$ & & -2.2212118764 & & -1.212931403003 & -0.000177913492 \\
\hline $\mathrm{C}$ & & -0.07390434131 & & -1.325170928228 & -0.002183154067 \\
\hline $\mathrm{C}$ & & 1.12027470968 & & -0.621617028220 & -0.002892399724 \\
\hline $\mathrm{H}$ & & $2.0745072779^{7}$ & & -1.138935705859 & -0.004033446347 \\
\hline $\mathrm{F}$ & & -0.05392321842 & 20 & -2.665096351527 & -0.002912978999 \\
\hline
\end{tabular}


11 major conf 0001

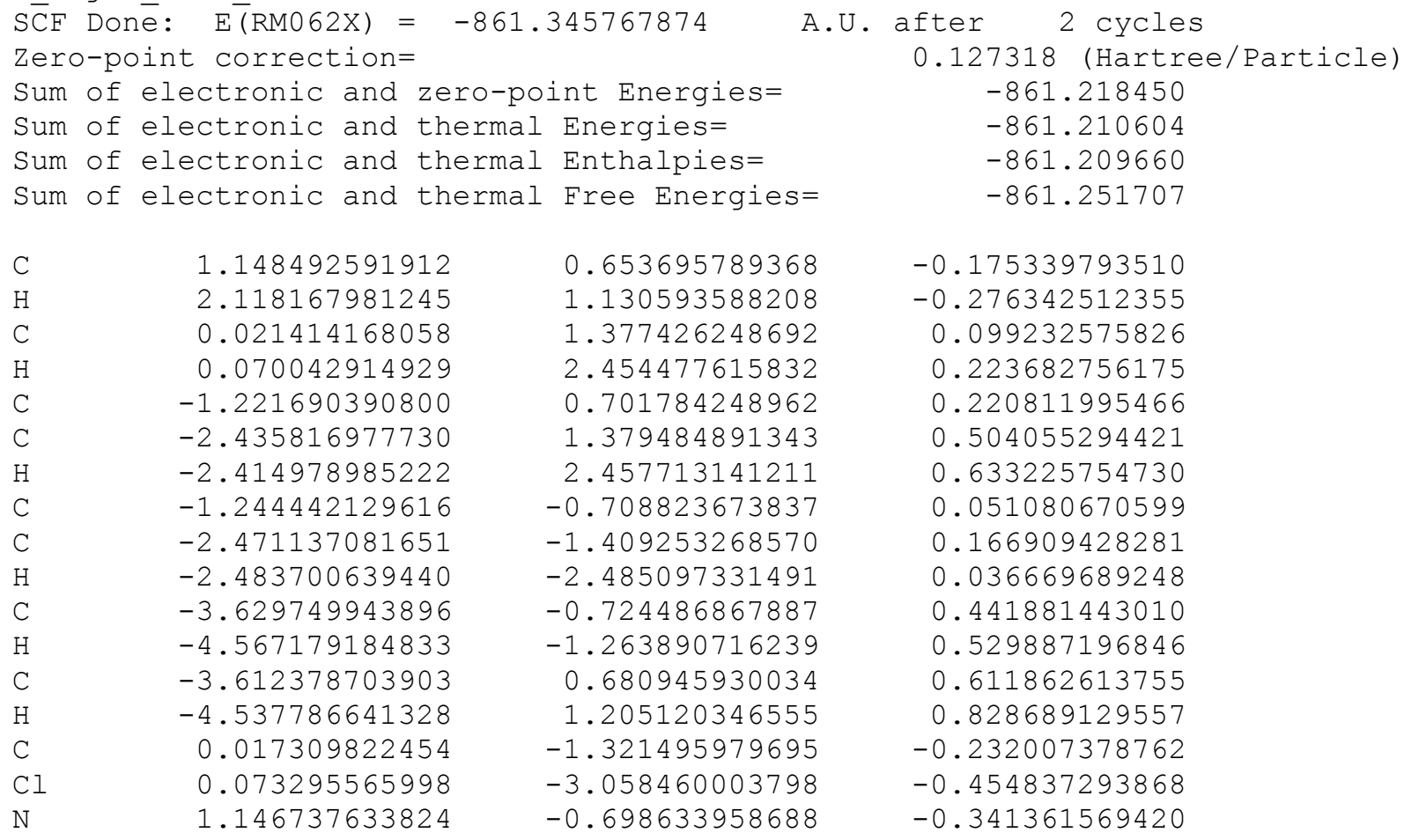


11 minor conf 0002

\begin{tabular}{|c|c|c|c|}
\hline \multicolumn{3}{|c|}{$\begin{array}{l}\text { SCF Done: } \\
\text { Zero-point }\end{array}$} & $\begin{array}{l}\text { after } 2 \text { cycles } \\
0.127151 \quad \text { (Hartree/Particle) }\end{array}$ \\
\hline Sum of & electronic and zero-po & int Energies= & -861.217188 \\
\hline Sum of & electronic and thermal & Energies $=$ & -861.209312 \\
\hline Sum of & electronic and thermal & Enthalpies $=$ & -861.208368 \\
\hline Sum of & electronic and thermal & Free Energies= & -861.250534 \\
\hline $\mathrm{C}$ & 1.140101602136 & 0.633244410949 & -0.170035474047 \\
\hline $\mathrm{Cl}$ & 2.690379524584 & 1.415778330527 & -0.319442797229 \\
\hline $\mathrm{C}$ & 0.028822745452 & 1.388108758862 & 0.108332472098 \\
\hline $\mathrm{H}$ & 0.102635229476 & 2.462564491093 & 0.236228377650 \\
\hline $\mathrm{C}$ & -1.216903822977 & 0.724372134833 & 0.223838289037 \\
\hline $\mathrm{C}$ & -2.435239485746 & 1.398161015077 & 0.509003703439 \\
\hline $\mathrm{H}$ & -2.424337779049 & 2.475383252134 & 0.647453499821 \\
\hline $\mathrm{C}$ & -1.237135378570 & -0.682813104830 & 0.043951429500 \\
\hline C & -2.460954390731 & -1.392774397283 & 0.149889878107 \\
\hline $\mathrm{H}$ & -2.459575028071 & -2.470353288463 & 0.009403024775 \\
\hline C & -3.622941737386 & -0.718858447347 & 0.425939946652 \\
\hline $\mathrm{H}$ & -4.561364004765 & -1.257381015649 & 0.507938725890 \\
\hline $\mathrm{C}$ & -3.605673798604 & 0.689397678629 & 0.606446178317 \\
\hline $\mathrm{H}$ & -4.534456249016 & 1.207483486666 & 0.824415995435 \\
\hline $\mathrm{C}$ & -0.003422327376 & -1.324740140525 & -0.239559383474 \\
\hline $\mathrm{H}$ & 0.016011246557 & -2.404393070441 & -0.383235901203 \\
\hline $\mathrm{N}$ & 1.146053654087 & -0.700180094232 & -0.344567964769 \\
\hline
\end{tabular}


13 major conf 0152

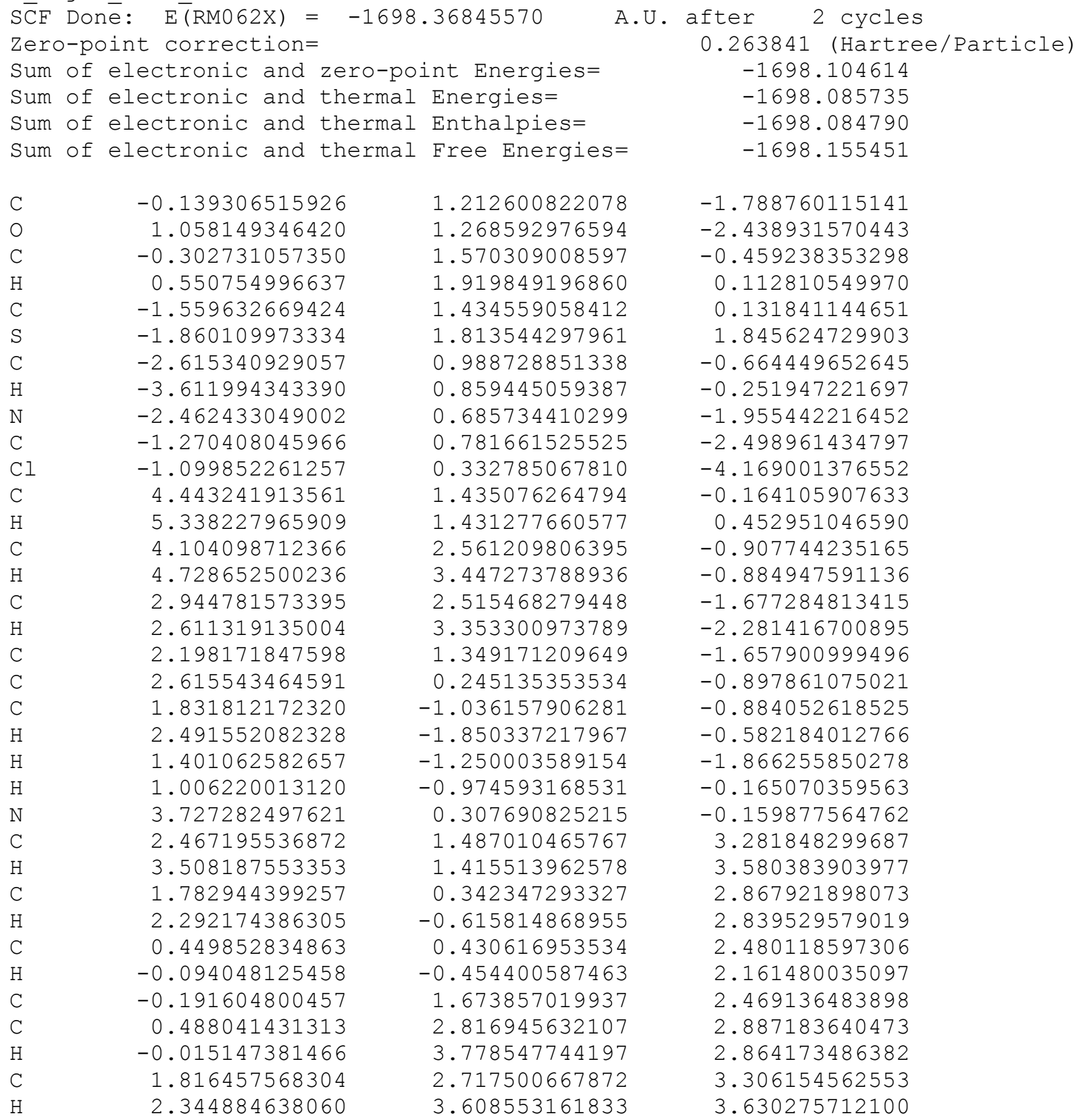


13 minor conf 0049

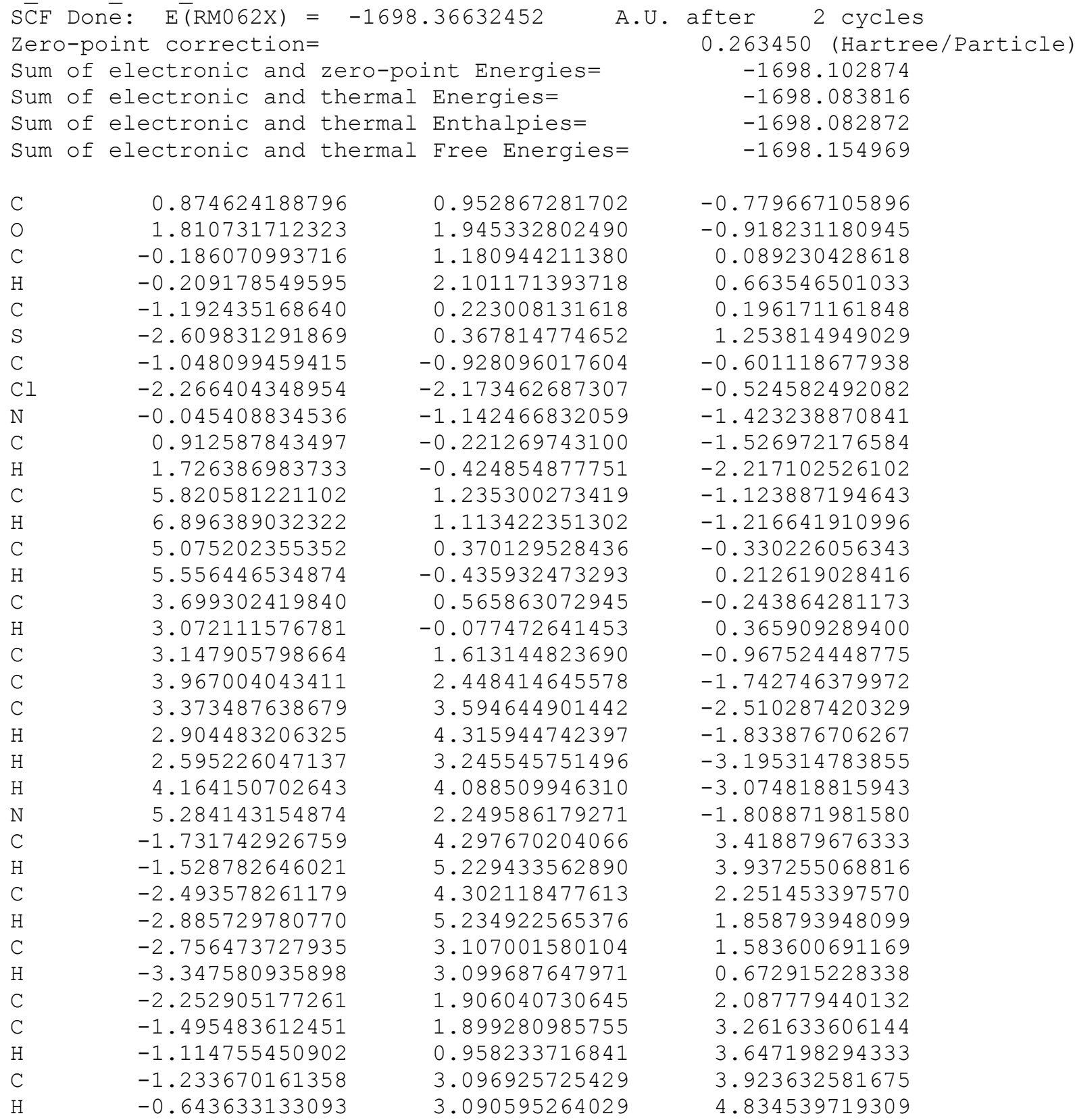




\begin{tabular}{|c|c|c|c|c|c|}
\hline \multicolumn{6}{|c|}{-3546.05890180} \\
\hline Zero & & oint correct & ion $=$ & & 0.142371 (Hartree/Particle) \\
\hline Sum & of & electronic & and zero-poj & int Energies $=$ & -3545.916531 \\
\hline Sum & of & electronic & and thermal & Energies $=$ & -3545.904133 \\
\hline Sum & $o f$ & electronic & and thermal & Enthalpies $=$ & -3545.903189 \\
\hline Sum & $o f$ & electronic & and thermal & Free Energies= & -3545.959105 \\
\hline $\mathrm{C}$ & & 0.390564 & 1072402 & 1.022339121066 & 1.095807653139 \\
\hline $\mathrm{C}$ & & -0.347904 & 4484371 & 1.741610730629 & 0.159036935036 \\
\hline $\mathrm{H}$ & & -0.315084 & $\$ 524021$ & 2.826568294035 & 0.164635317681 \\
\hline $\mathrm{C}$ & & -1.115500 & 489199 & 1.049867043813 & -0.764323866973 \\
\hline $\mathrm{Br}$ & & -2.126723 & 3772371 & 1.990578455262 & -2.029280701190 \\
\hline $\mathrm{C}$ & & -1.103086 & 5157221 & -0.353494463524 & -0.708195949387 \\
\hline $\mathrm{Cl}$ & & -2.028584 & 9927174 & -1.287621265067 & -1.834558152583 \\
\hline $\mathrm{N}$ & & -0.404966 & 5095039 & -1.038057174214 & 0.178029306748 \\
\hline $\mathrm{C}$ & & 0.328883 & 3639469 & -0.369621724990 & 1.067222146786 \\
\hline $\mathrm{H}$ & & 0.887565 & 5256312 & -0.964838128451 & 1.782161221285 \\
\hline $\mathrm{C}$ & & 1.212731 & 459881 & 1.776964217546 & 2.085509041314 \\
\hline O & & 1.859108 & 3665909 & 0.964913815720 & 2.928484801574 \\
\hline $\mathrm{C}$ & & 2.691444 & 1161516 & 1.601879604020 & 3.919082720565 \\
\hline $\mathrm{C}$ & & 4.034383 & 3041435 & 1.988165472280 & 3.330073954879 \\
\hline $\mathrm{H}$ & & 4.670429 & 9181480 & 2.411080996289 & 4.112640859426 \\
\hline $\mathrm{H}$ & & 3.908412 & 2634296 & 2.738372641222 & 2.546480245722 \\
\hline $\mathrm{H}$ & & 4.537099 & 9336164 & 1.112083146620 & 2.912687434465 \\
\hline $\mathrm{H}$ & & 2.793764 & 1870882 & 0.851177541167 & 4.704041518177 \\
\hline $\mathrm{H}$ & & 2.160399 & 9914383 & 2.472763513233 & 4.310355781150 \\
\hline O & & 1.276064 & \pm 215267 & 2.984268163342 & 2.115109732188 \\
\hline
\end{tabular}


15 minor conf 0004

\begin{tabular}{|c|c|c|c|}
\hline \multicolumn{4}{|c|}{ SCF Done: $\quad \bar{E}(\mathrm{RM} 062 \mathrm{X})=$} \\
\hline & -point correction= & & 0.142265 (Hartree/Particle) \\
\hline Sum & of electronic and zero-po & int Energies= & -3545.910102 \\
\hline Sum & of electronic and thermal & Energies= & -3545.897646 \\
\hline Sum & of electronic and thermal & Enthalpies= & -3545.896702 \\
\hline Sum & of electronic and thermal & Free Energies= & -3545.951840 \\
\hline $\mathrm{C}$ & 0.806071009320 & 0.701444471721 & 0.159893200515 \\
\hline $\mathrm{C}$ & 0.109242486099 & 1.893366054346 & 0.357270356912 \\
\hline $\mathrm{H}$ & 0.654013335290 & 2.767835773750 & 0.698333388336 \\
\hline $\mathrm{C}$ & -1.253204440213 & 1.930303169764 & 0.111443431832 \\
\hline $\mathrm{Br}$ & -2.241157910266 & 3.511205645542 & 0.329249388165 \\
\hline $\mathrm{C}$ & -1.889281864408 & 0.766192298214 & -0.309018742607 \\
\hline $\mathrm{H}$ & -2.955493068247 & 0.758077397791 & -0.517039866442 \\
\hline $\mathrm{N}$ & -1.238296438818 & -0.382665475275 & -0.469728862800 \\
\hline $\mathrm{C}$ & 0.063308759405 & -0.419664205549 & -0.245522474242 \\
\hline $\mathrm{Cl}$ & 0.780106006504 & -1.991962199058 & -0.428401343297 \\
\hline $\mathrm{C}$ & 2.283717880127 & 0.774526660620 & 0.414213990965 \\
\hline 0 & 2.995687496682 & -0.088114764045 & -0.300545558117 \\
\hline C & 4.422088744666 & -0.046012555927 & -0.098091986095 \\
\hline $\mathrm{C}$ & 5.026373896951 & -1.101159069678 & -0.996998436904 \\
\hline $\mathrm{H}$ & 4.639504516843 & -2.090827521489 & -0.742928778371 \\
\hline $\mathrm{H}$ & 4.792339670880 & -0.895190065241 & -2.044123808559 \\
\hline $\mathrm{H}$ & 6.112809666601 & -1.109317716551 & -0.878236897308 \\
\hline $\mathrm{H}$ & 4.624903224881 & -0.232475957782 & 0.960329836339 \\
\hline $\mathrm{H}$ & 4.775800482476 & 0.960717134671 & -0.338630219775 \\
\hline 0 & 2.762466545227 & 1.592720924176 & 1.166533381455 \\
\hline
\end{tabular}


17 major conf 0001

\begin{tabular}{|c|c|c|c|}
\hline \multicolumn{4}{|c|}{ SCF Done: $\quad \mathrm{E}(\mathrm{RM} 062 \mathrm{X})=$} \\
\hline \multicolumn{3}{|c|}{ o-point correction= } & 0.247676 (Hartree/Particle) \\
\hline Sum & of electronic and zero-po: & int Energies= & -1642.782428 \\
\hline Sum & of electronic and thermal & Energies= & -1642.764931 \\
\hline Sum & of electronic and thermal & Enthalpies= & -1642.763987 \\
\hline Sum & of electronic and thermal & Free Energies= & -1642.833199 \\
\hline $\mathrm{C}$ & 0.967662219917 & 0.249311825772 & -0.681637480661 \\
\hline 0 & 2.096633581187 & 0.748848420998 & -1.265248265810 \\
\hline $\mathrm{c}$ & 0.091008714860 & 1.109746076147 & -0.039831267115 \\
\hline $\mathrm{H}$ & 0.345547828356 & 2.163495758865 & 0.028125808989 \\
\hline $\mathrm{C}$ & -1.094654254333 & 0.596213387748 & 0.488663997151 \\
\hline $\mathrm{S}$ & -2.285435390222 & 1.569803108380 & 1.379706074348 \\
\hline $\mathrm{C}$ & -1.331223357275 & -0.773461534597 & 0.349464535110 \\
\hline $\mathrm{H}$ & -2.244782270308 & -1.219115885568 & 0.734687335919 \\
\hline & -0.481405238091 & -1.602592378365 & -0.258761044868 \\
\hline C & 0.628414707060 & -1.109170702266 & -0.764729131600 \\
\hline $\mathrm{Cl}$ & 1.702580756263 & -2.203118881898 & -1.580442710411 \\
\hline C & 5.844296242958 & 0.266906411147 & 0.474910340722 \\
\hline $\mathrm{H}$ & 6.831434523431 & 0.154507234730 & 0.910234722853 \\
\hline $\mathrm{C}$ & 5.696799527074 & 0.791349277271 & -0.810442648457 \\
\hline $\mathrm{H}$ & 6.570575595481 & 1.090322422847 & -1.380845250113 \\
\hline $\mathrm{C}$ & 4.433992768105 & 0.932807976661 & -1.373806324287 \\
\hline $\mathrm{H}$ & 4.294257940886 & 1.333300598377 & -2.372092334765 \\
\hline $\mathrm{C}$ & 3.313780422683 & 0.547678780888 & -0.640787946238 \\
\hline $\mathrm{C}$ & 3.438310795077 & 0.025088452631 & 0.643084501016 \\
\hline $\mathrm{H}$ & 2.562349317953 & -0.274624348849 & 1.208585222269 \\
\hline $\mathrm{C}$ & 4.714148560287 & -0.114332573058 & 1.191485352711 \\
\hline $\mathrm{H}$ & 4.815981727415 & -0.526850848882 & 2.190351698893 \\
\hline $\mathrm{C}$ & -1.546134445679 & 5.739827013268 & -0.343824084603 \\
\hline H & -1.375065249593 & 6.732175375118 & -0.748897131407 \\
\hline $\mathrm{C}$ & -1.224640546452 & 5.464084303405 & 0.982743501702 \\
\hline $\mathrm{H}$ & -0.801396518369 & 6.238981898727 & 1.613940233766 \\
\hline $\mathrm{C}$ & -1.435811629515 & 4.186776060568 & 1.503148280058 \\
\hline $\mathrm{H}$ & -1.174081538902 & 3.957273954035 & 2.531370914033 \\
\hline $\mathrm{C}$ & -1.983481521470 & 3.191131803500 & 0.693698459795 \\
\hline $\mathrm{C}$ & -2.318747099023 & 3.469459642611 & -0.634908994642 \\
\hline $\mathrm{H}$ & -2.751669670766 & 2.689000536794 & -1.253782284585 \\
\hline $\mathrm{C}$ & -2.090111007092 & 4.740707927758 & -1.152537935638 \\
\hline $\mathrm{H}$ & -2.345035491905 & 4.954668905240 & -2.185626144134 \\
\hline
\end{tabular}


17 minor conf 0008

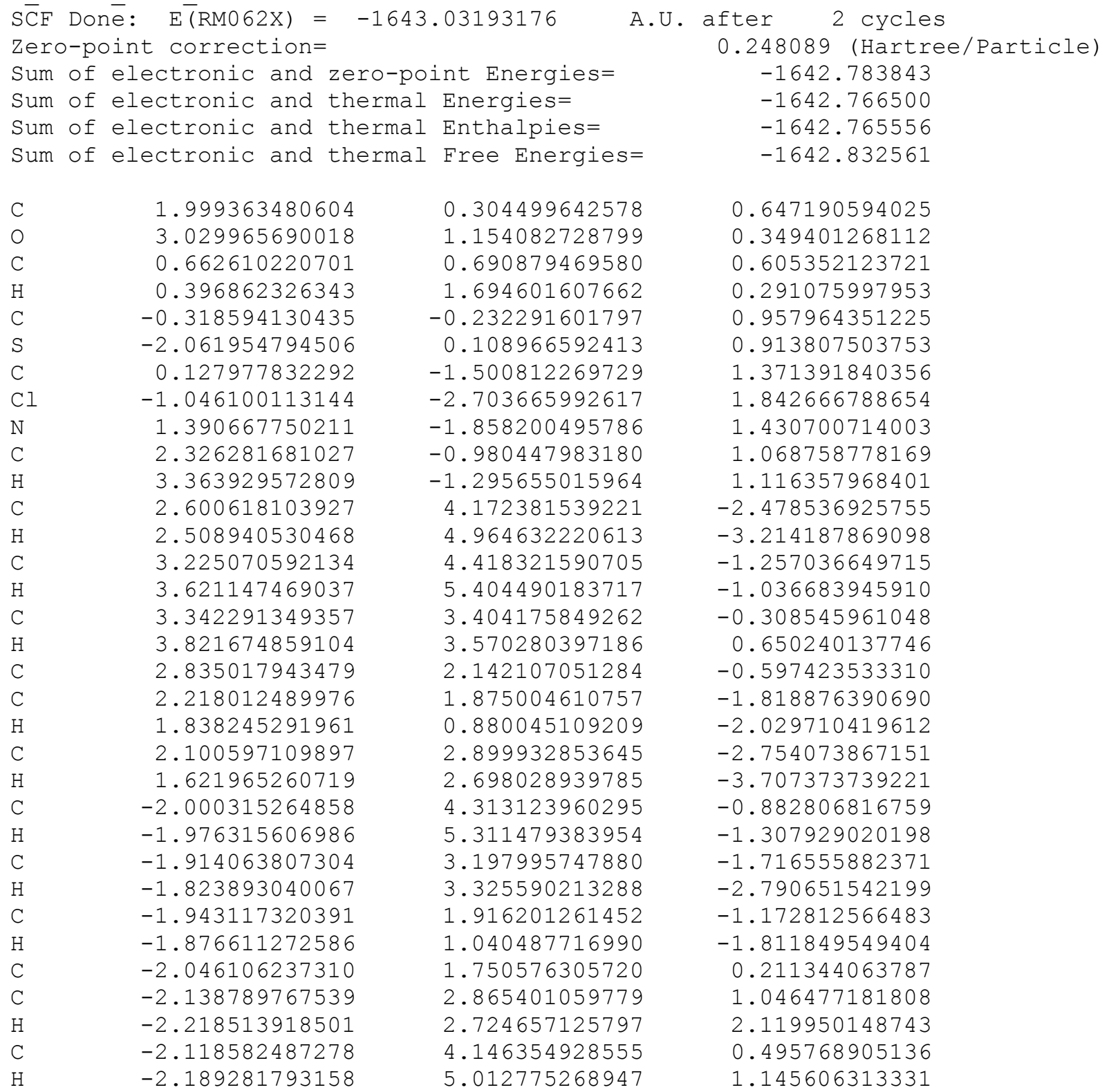




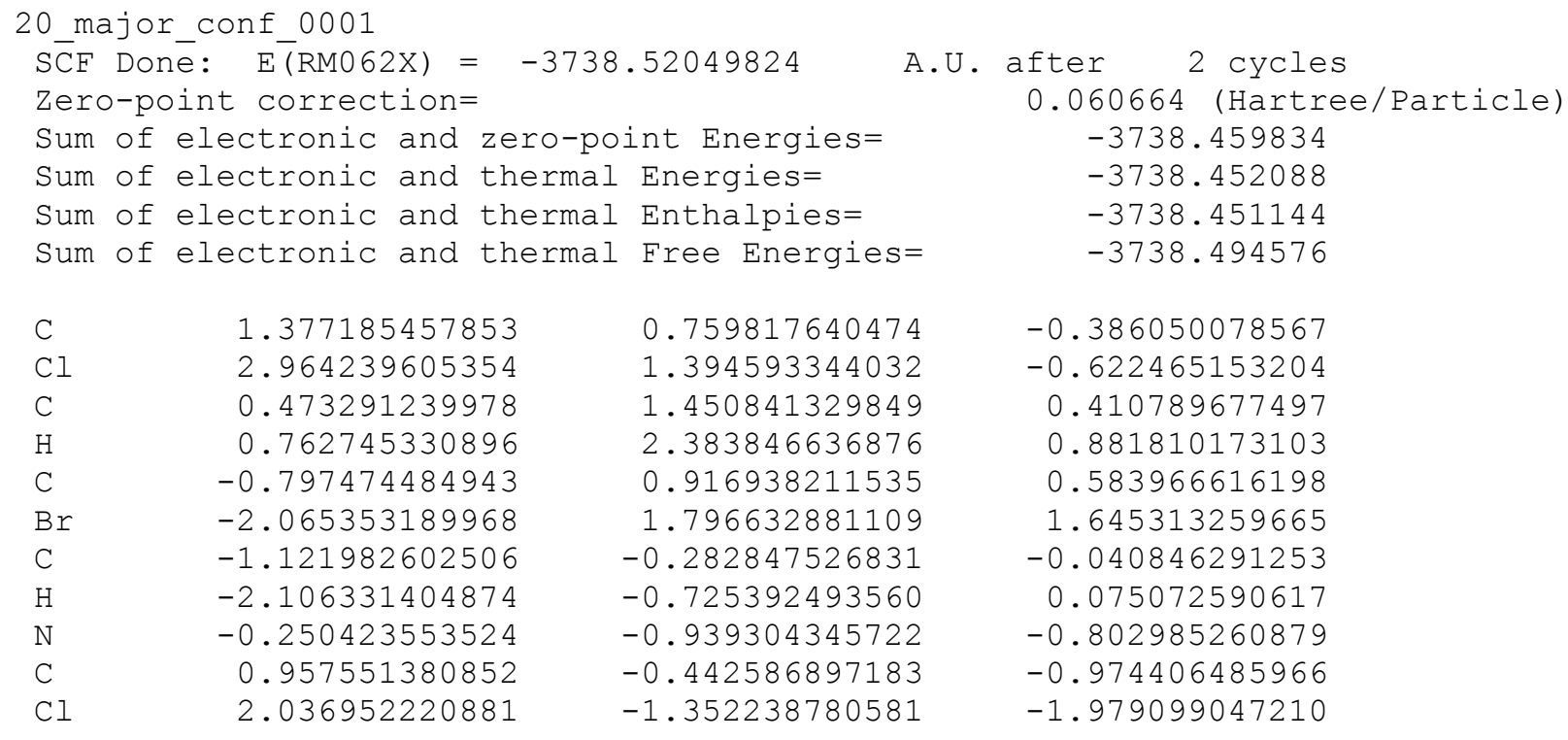




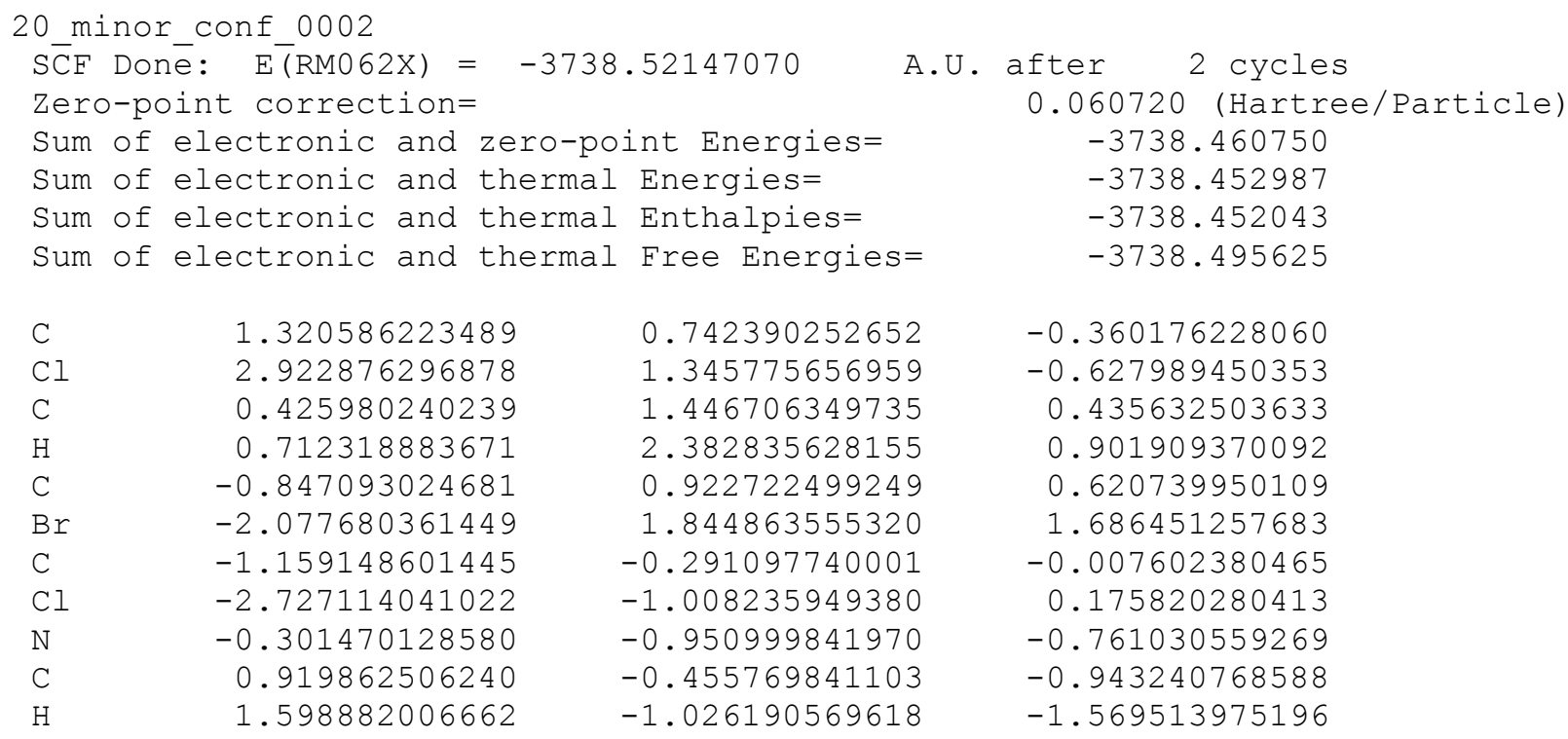


S8. Electrostatic potential-derived charges (using the Merz-Kollman-Singh and CHELPG schemes, the M06-2X/6-31+G(d,p) density, and constrained to reproduce dipole moment) for substrate 7 and resultant major and minor deprotonated intermediates.

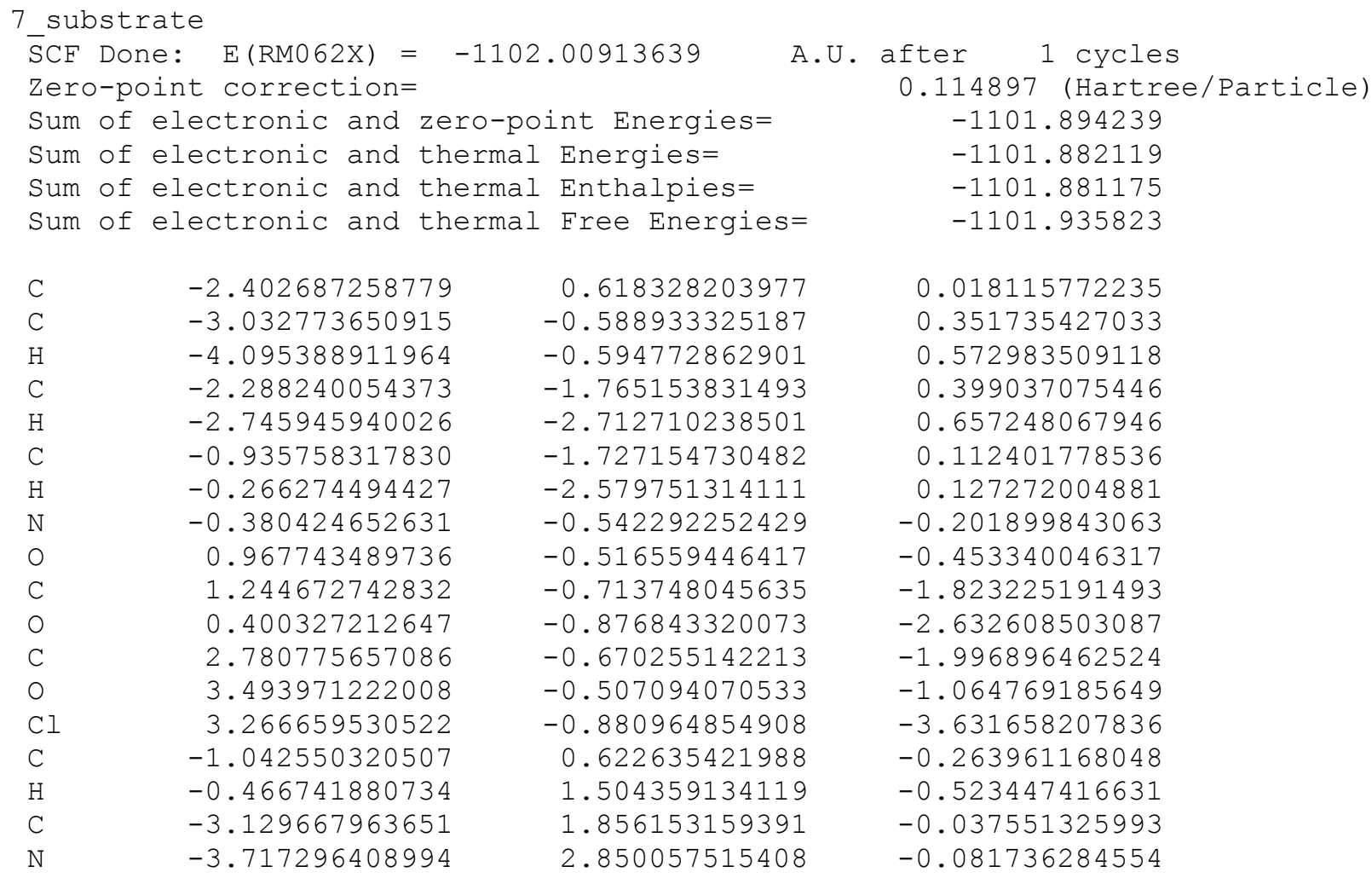

MK Scheme:

$\begin{array}{rlr}1 & \mathrm{C} & 0.103363 \\ 2 & \mathrm{C} & -0.055005 \\ 3 & \mathrm{H} & 0.213716 \\ 4 & \mathrm{C} & -0.096320 \\ 5 & \mathrm{H} & 0.214826 \\ 6 & \mathrm{C} & -0.136136 \\ 7 & \mathrm{H} & 0.252421 \\ 8 & \mathrm{~N} & 0.509509 \\ 9 & \mathrm{O} & -0.385656 \\ 10 & \mathrm{C} & 0.665821 \\ 11 & \mathrm{O} & -0.418535 \\ 12 & \mathrm{C} & 0.271016 \\ 13 & \mathrm{O} & -0.258705 \\ 14 & \mathrm{C} & 0.046082 \\ 15 & \mathrm{C} & -0.172273 \\ 16 & \mathrm{H} & 0.267109 \\ 17 & \mathrm{C} & 0.258797 \\ 18 & \mathrm{~N} & -0.280030\end{array}$


CHELPG Scheme:
C $\quad 0.075052$
C $\quad 0.005983$
$\mathrm{H} \quad 0.178183$
C $\quad-0.100930$
$\mathrm{H} \quad 0.191778$
C $\quad-0.021906$
H $\quad 0.210799$
N $\quad 0.374825$
O $\quad-0.373360$
C $\quad 0.716255$
O $\quad-0.453262$
C $\quad 0.314626$
o $\quad-0.289870$
Cl 0.033219
C $\quad-0.073068$
H $\quad 0.227996$
C $\quad 0.283369$
N $\quad-0.299691$ 
7_major_conf_0016

MK Scheme:

$\begin{array}{rrr}1 & \mathrm{C} & 0.577604 \\ 2 & \mathrm{C} & -0.520547 \\ 3 & \mathrm{H} & 0.238691 \\ 4 & \mathrm{C} & 0.181167 \\ 5 & \mathrm{H} & 0.129329 \\ 6 & \mathrm{C} & -0.579217 \\ 7 & \mathrm{H} & 0.278984 \\ 8 & \mathrm{~N} & 0.994971 \\ 9 & \mathrm{O} & -0.488768 \\ 10 & \mathrm{C} & 0.668641 \\ 11 & \mathrm{O} & -0.451243 \\ 12 & \mathrm{C} & 0.327535 \\ 13 & \mathrm{O} & -0.302879 \\ 14 & \mathrm{C} 1 & -0.062501 \\ 15 & \mathrm{C} & -0.805550 \\ 16 & \mathrm{C} & 0.224435 \\ 17 & \mathrm{~N} & -0.410652\end{array}$

CHELPG Scheme:

$\begin{array}{rrr}1 & \mathrm{C} & 0.490091 \\ 2 & \mathrm{C} & -0.391980 \\ 3 & \mathrm{H} & 0.194400 \\ 4 & \mathrm{C} & 0.126154 \\ 5 & \mathrm{H} & 0.107616 \\ 6 & \mathrm{C} & -0.405633 \\ 7 & \mathrm{H} & 0.232843 \\ 8 & \mathrm{~N} & 0.861008 \\ 9 & \mathrm{O} & -0.524030 \\ 10 & \mathrm{C} & 0.723710 \\ 11 & \mathrm{O} & -0.485756 \\ 12 & \mathrm{C} & 0.384528 \\ 13 & \mathrm{O} & -0.336744 \\ 14 & \mathrm{C} 1 & -0.082200 \\ 15 & \mathrm{C} & -0.708046 \\ 16 & \mathrm{C} & 0.245229 \\ 17 & \mathrm{~N} & -0.431190\end{array}$


7_minor_conf_0002

MK Scheme:

$\begin{array}{rlr}1 & \mathrm{C} & 0.367288 \\ 2 & \mathrm{C} & -0.569541 \\ 3 & \mathrm{H} & 0.248355 \\ 4 & \mathrm{C} & 0.473216 \\ 5 & \mathrm{H} & 0.044657 \\ 6 & \mathrm{C} & -0.850596 \\ 7 & \mathrm{~N} & 1.087040 \\ 8 & \mathrm{O} & -0.508450 \\ 9 & \mathrm{C} & 0.654899 \\ 10 & \mathrm{O} & -0.445818 \\ 11 & \mathrm{C} & 0.351018 \\ 12 & \mathrm{O} & -0.310169 \\ 13 & \mathrm{C} & -0.071137 \\ 14 & \mathrm{C} & -0.660326 \\ 15 & \mathrm{H} & 0.305443 \\ 16 & \mathrm{C} & 0.291217 \\ 17 & \mathrm{~N} & -0.407095\end{array}$

CHELPG Scheme:

$\begin{array}{rlr}1 & \mathrm{C} & 0.278051 \\ 2 & \mathrm{C} & -0.446839 \\ 3 & \mathrm{H} & 0.200347 \\ 4 & \mathrm{C} & 0.461040 \\ 5 & \mathrm{H} & -0.003366 \\ 6 & \mathrm{C} & -0.743862 \\ 7 & \mathrm{~N} & 0.907805 \\ 8 & \mathrm{O} & -0.536588 \\ 9 & \mathrm{C} & 0.723853 \\ 10 & \mathrm{O} & -0.484154 \\ 11 & \mathrm{C} & 0.389163 \\ 12 & \mathrm{O} & -0.336949 \\ 13 & \mathrm{Cl} & -0.086219 \\ 14 & \mathrm{C} & -0.460542 \\ 15 & \mathrm{H} & 0.252793 \\ 16 & \mathrm{C} & 0.300913 \\ 17 & \mathrm{~N} & -0.415446\end{array}$


S9. Product ratios and relative free energies of intermediates in Table 2, $\mathrm{CH}_{2} \mathrm{Cl}_{2}$ solvent.

\begin{tabular}{|c|c|c|c|}
\hline entry & major product & regioselectivity & $\begin{array}{r}\Delta \mathrm{G}_{\mathrm{rel}} \text { (minor-ma } \\
\text { intermediat } \\
\quad(\mathrm{kcal} / \mathrm{mol})^{\mathrm{a}}\end{array}$ \\
\hline 2 & & 93.6:6.4 & $\begin{array}{c}+1.7 \\
(+1.2)\end{array}$ \\
\hline 6 & & $77.9: 22.1$ & $\begin{array}{c}+1.0 \\
(+1.8)\end{array}$ \\
\hline 7 & & 100:0 & $\begin{array}{c}+3.0 \\
(+4.6)\end{array}$ \\
\hline 9 & & 100:0 & $\begin{array}{c}+3.4 \\
(+2.4)\end{array}$ \\
\hline 11 & $\mathrm{Cl}$ & $100: 0$ & $\begin{array}{c}+8.2 \\
(+8.3)\end{array}$ \\
\hline 13 & & $100: 0$ & $\begin{array}{c}+2.9 \\
(+2.5)\end{array}$ \\
\hline 15 & & 99.4:0.6 & $\begin{array}{l}+3.7 \\
(+4.6)\end{array}$ \\
\hline 17 & & $98.3: 1.7$ & $\begin{array}{c}+1.9 \\
(+1.6)\end{array}$ \\
\hline 20 & & $73.5: 26.5$ & $\begin{array}{c}+0.2 \\
(+1.8)\end{array}$ \\
\hline
\end{tabular}

${ }^{a}$ from IEFPCM-M06-2X/6-31+G(d,p) re-optimization and frequency determination of the gas-phase M06-2X/6-31+G(d,p) conformer possessing lowest Gibbs free energy at $298 \mathrm{~K}$. ${ }^{b}$ Positive values indicate greater stability of the intermediate or final product corresponding to that found experimentally. Parenthesized values correspond to implicit IEFPCM dichloromethane solvent; un-parenthesized values correspond to gas-phase values reported in Table 2. 


\section{S10. IEFPCM-M06-2X/6-31+G(d,p) coordinates and energies of major and minor intermediates for species $2,6,7,9,11,13,15,17$, and $20, \mathrm{CH}_{2} \mathrm{Cl}_{2}$ solvent.}

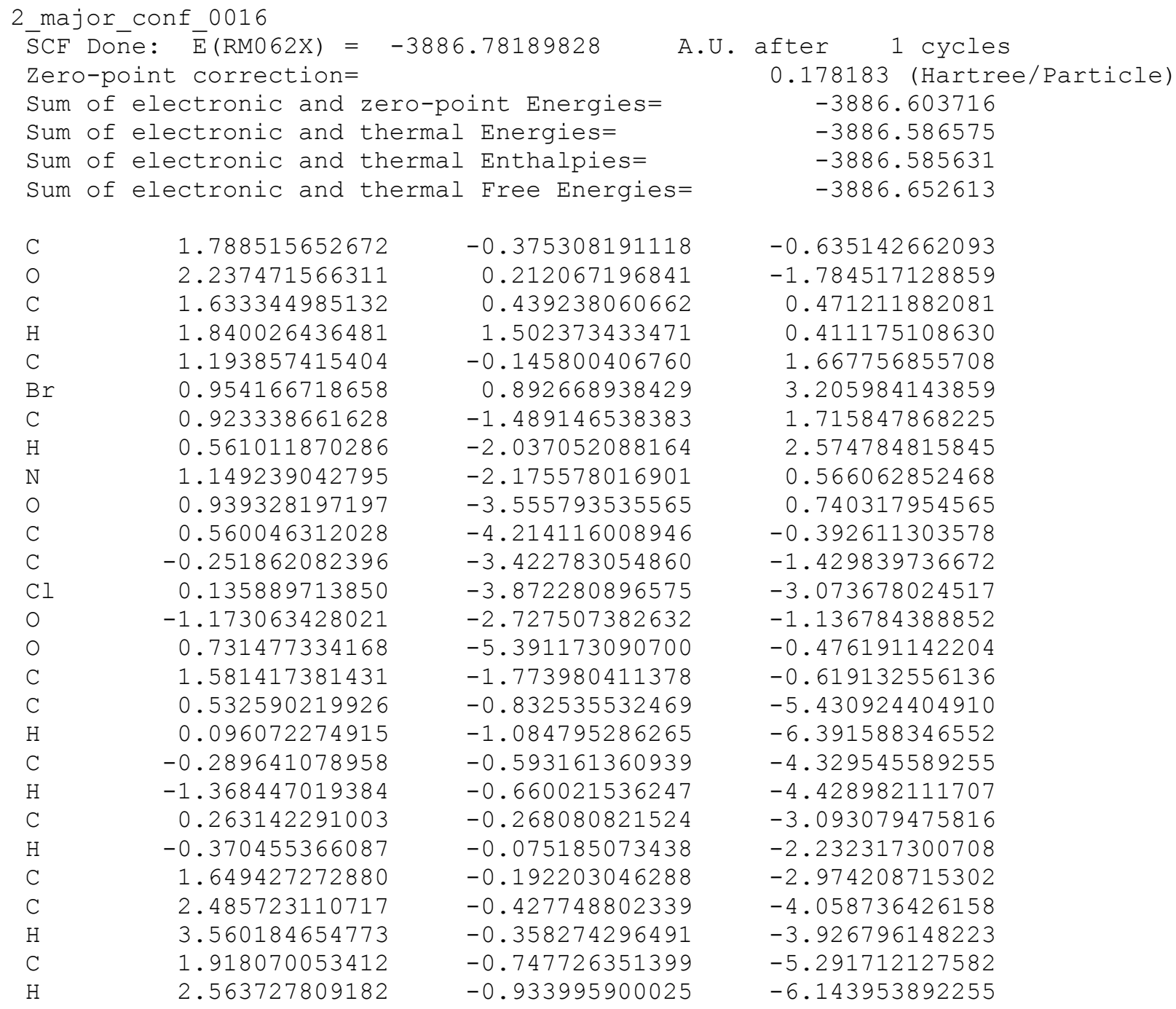




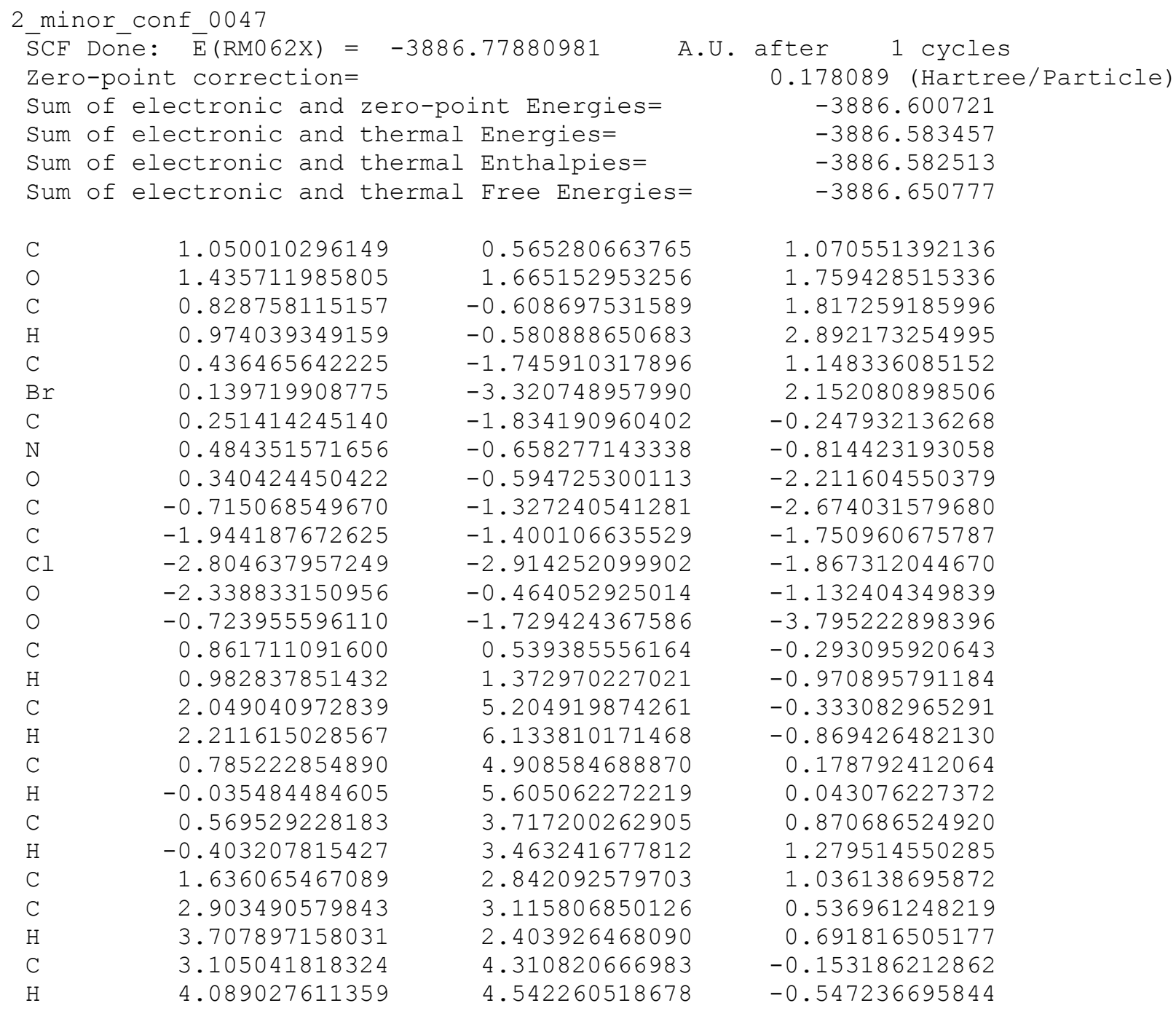




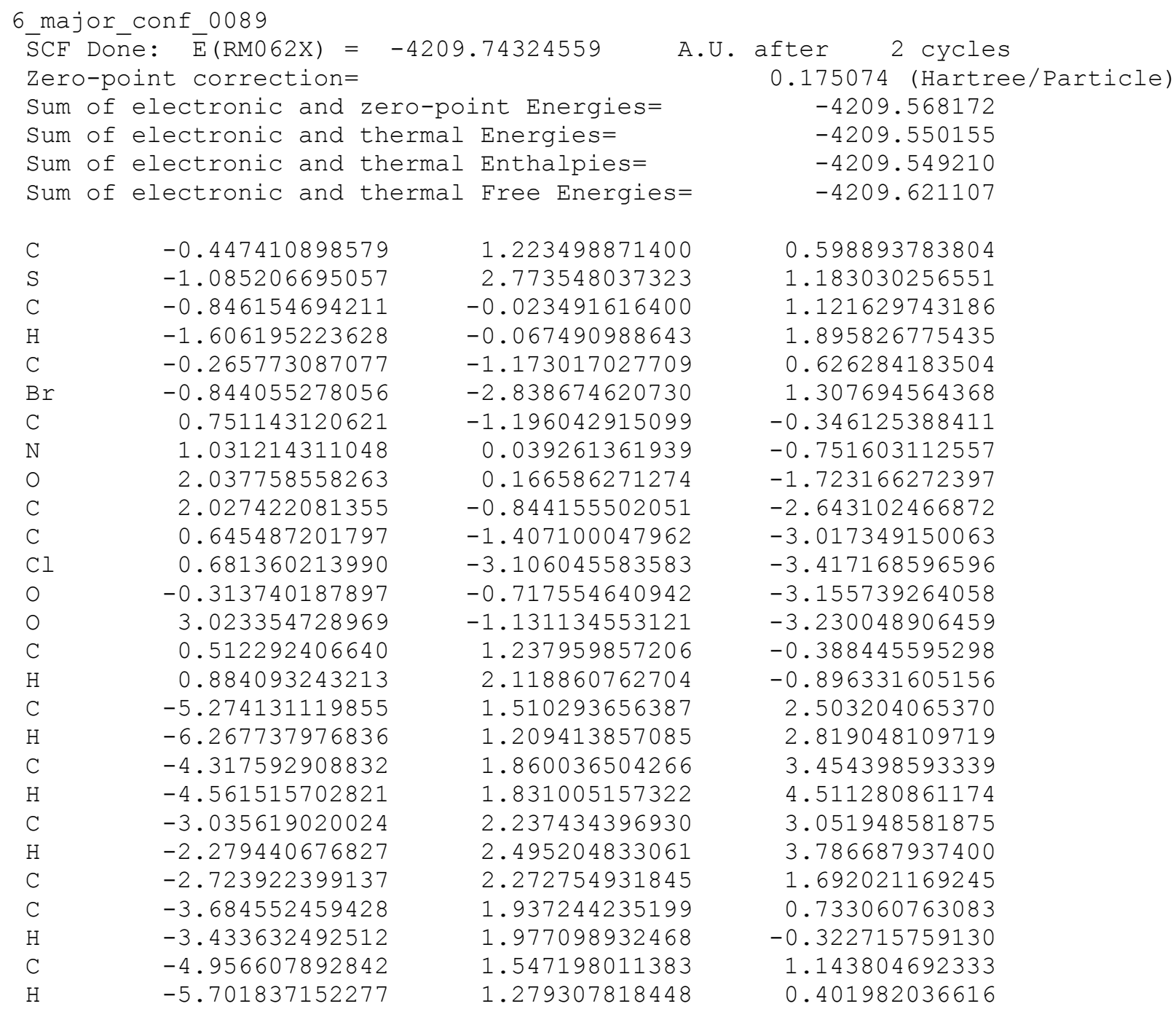




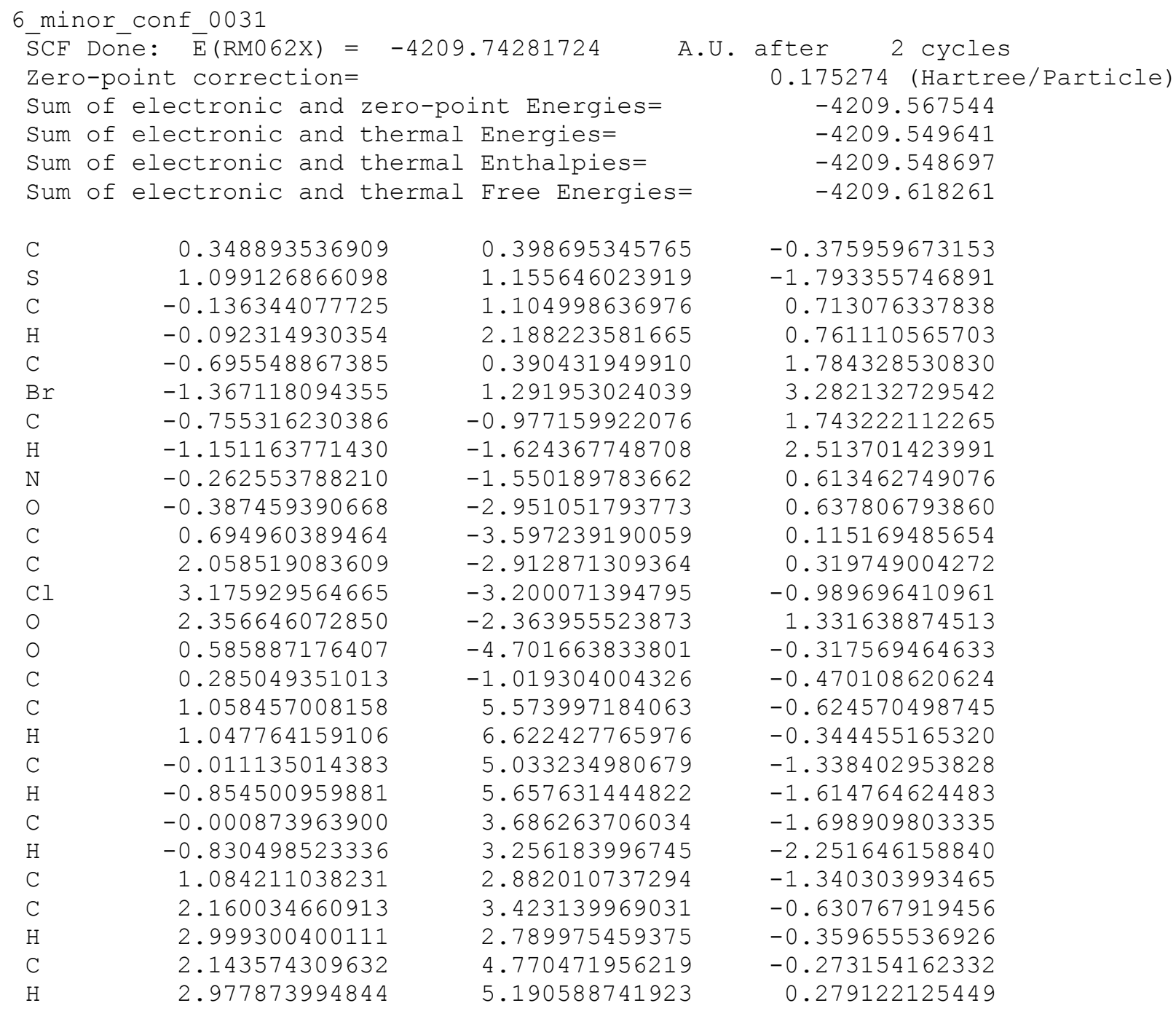




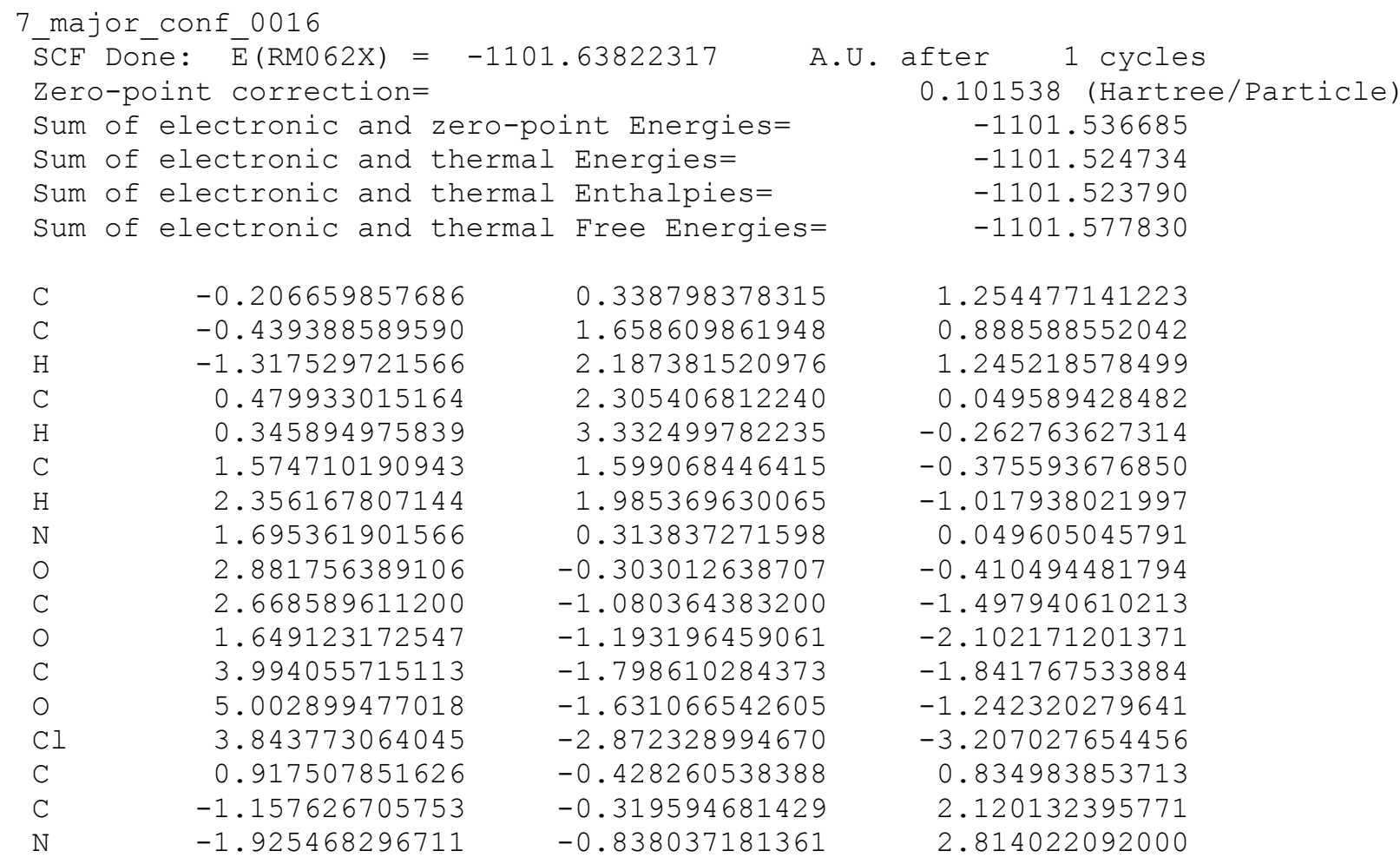




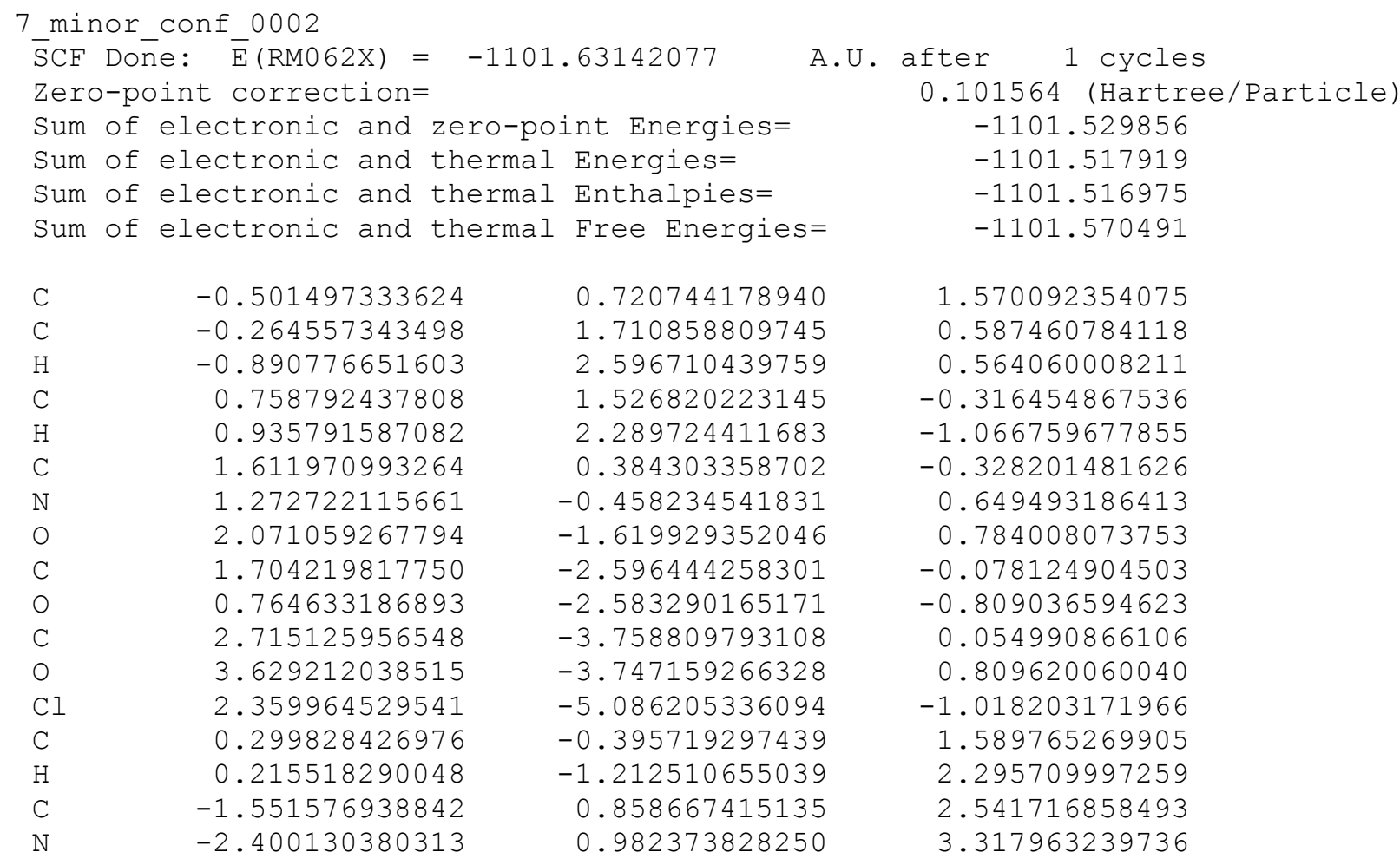




\begin{tabular}{|c|c|c|c|c|c|}
\hline \multicolumn{6}{|c|}{ _major_conf_o038 } \\
\hline $\bar{S} C F$ & Don & $\overline{\mathrm{E}}(\mathrm{RM} 062 \mathrm{X})=$ & -3679 & 9.83090118 & after 1 cycles \\
\hline Zerc & & oint correction= & & & 0.085006 (Hartree/Particle \\
\hline Sum & of & electronic and $\mathrm{z}$ & zero-po & oint Energies= & -3679.745895 \\
\hline Sum & of & electronic and $t$ & thermal & 1 Energies= & -3679.733602 \\
\hline Sum & of & electronic and $t$ & thermal & Enthalpies= & -3679.732658 \\
\hline Sum & of & electronic and $t$ & thermal & 1 Free Energies= & -3679.788342 \\
\hline $\mathrm{C}$ & & -0.88830507878 & & -0.365254548398 & 0.795514603494 \\
\hline $\mathrm{F}$ & & -1.99389535231 & & -1.034649743986 & 1.175421873250 \\
\hline $\mathrm{C}$ & & -0.70423500534 & & 0.894283304772 & 1.315432253049 \\
\hline $\mathrm{H}$ & & -1.41958316186 & & 1.332810419467 & 2.002969341063 \\
\hline $\mathrm{C}$ & & 0.45070582221 & & 1.591448152699 & 0.922364346162 \\
\hline $\mathrm{Br}$ & & 0.79999918929 & & 3.309336555396 & 1.569637109957 \\
\hline $\mathrm{C}$ & & 1.32614946467 & & 0.999864175380 & 0.050799660125 \\
\hline $\mathrm{H}$ & & 2.24518636317 & & 1.439913509071 & -0.312231848161 \\
\hline $\mathrm{N}$ & & 1.00120370249 & & -0.250957174056 & -0.375286314436 \\
\hline 0 & & 1.98221901637 & & -0.793740075264 & -1.238570914628 \\
\hline $\mathrm{C}$ & & 1.65465681935 & & -0.641172572739 & -2.543681064896 \\
\hline 0 & & 0.71849396341 & & -0.046553794732 & -2.975850039034 \\
\hline $\mathrm{C}$ & & 2.72446097515 & & -1.364034842030 & -3.393959054844 \\
\hline 0 & & 3.67609932713 & & -1.897776557786 & -2.930618961871 \\
\hline $\mathrm{Cl}$ & & 2.38267140815 & & -1.300383798740 & -5.102044576814 \\
\hline $\mathrm{C}$ & & -0.03432745313 & & -1.030933009053 & -0.100996412419 \\
\hline
\end{tabular}




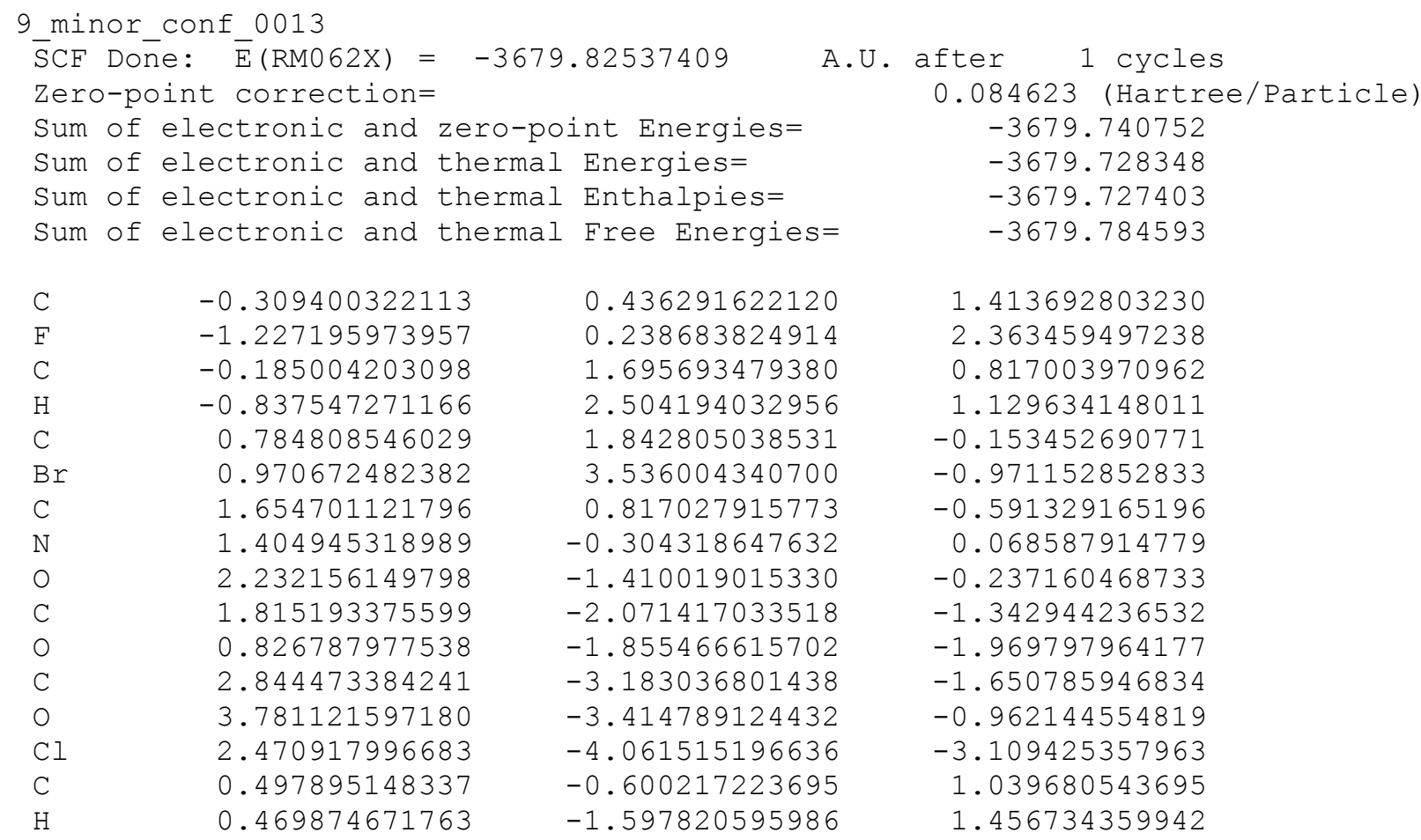


11 major conf 0013

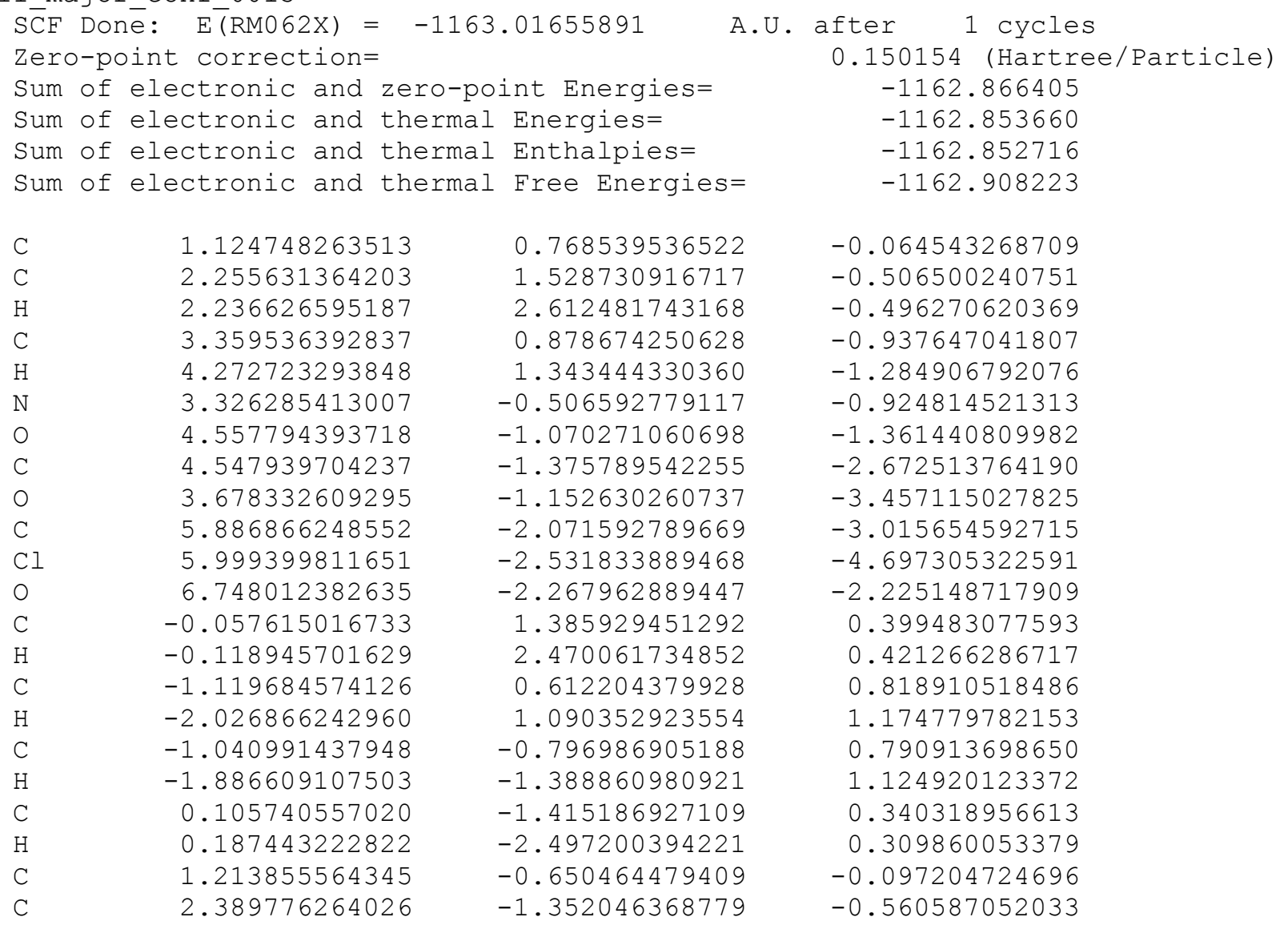


11 minor conf 0031

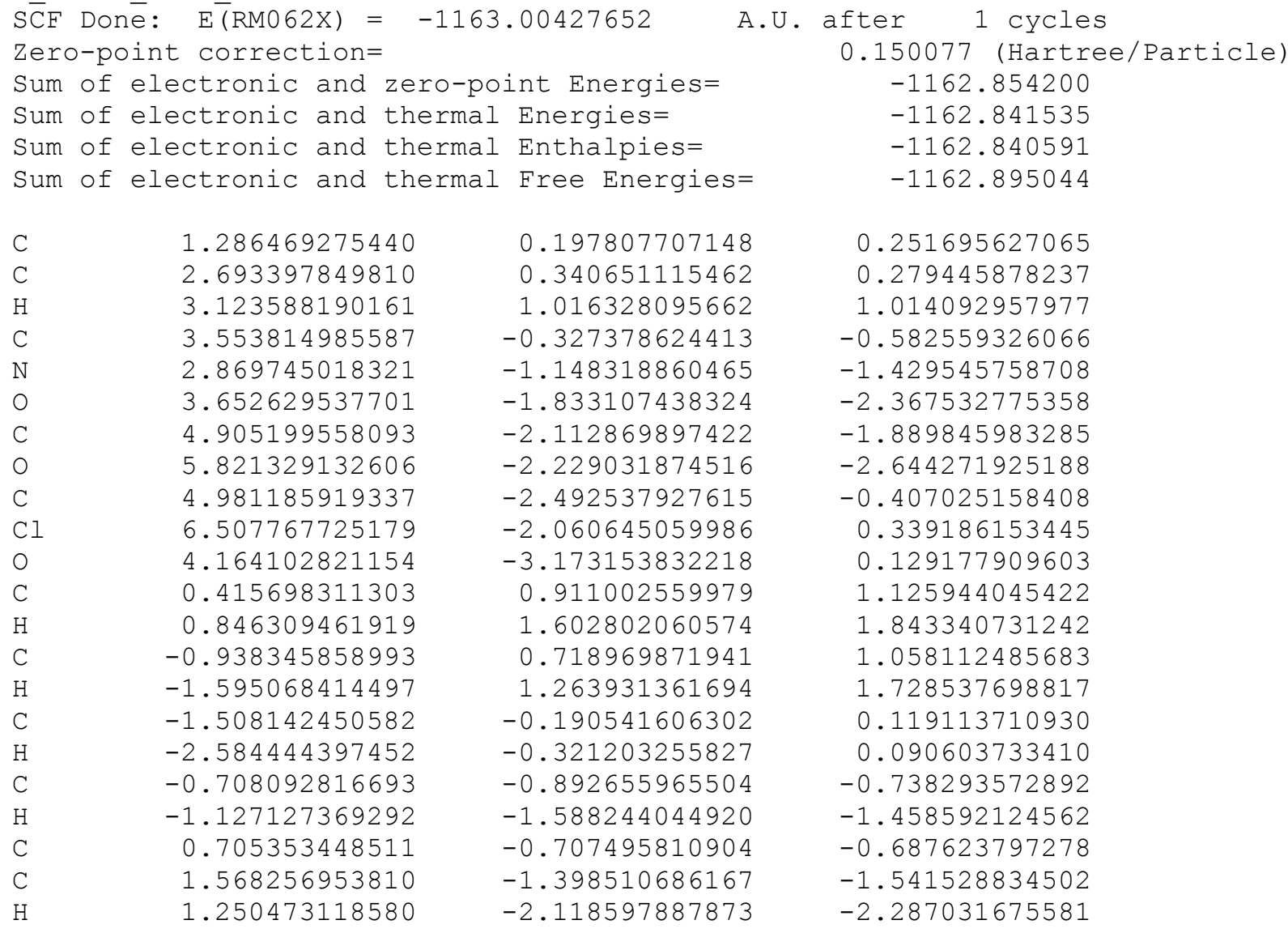


13 major conf 0098

$\begin{array}{lc}\mathrm{S} \bar{C} F \text { Done: } \bar{E}(\mathrm{RM} 06 \mathrm{X})=-2000.04152443 \quad \text { A.U. after } & 2 \text { cycles } \\ \text { Zero-point correction= } & 0.286593 \text { (Hartree/Particle) } \\ \text { Sum of electronic and zero-point Energies= } & -1999.754932 \\ \text { Sum of electronic and thermal Energies= } & -1999.731265 \\ \text { Sum of electronic and thermal Enthalpies= } & -1999.730321 \\ \text { Sum of electronic and thermal Free Energies= } & -1999.813284\end{array}$

\begin{tabular}{|c|c|c|c|}
\hline $\mathrm{C}$ & -0.006373361904 & -0.402417281695 & -0.979939178782 \\
\hline O & 0.359031063536 & -1.713688540626 & -1.120783464919 \\
\hline C & -1.367210432427 & -0.151399075821 & -0.974547785688 \\
\hline $\mathrm{H}$ & -2.074143273897 & -0.970863435590 & -1.068242597212 \\
\hline $\mathrm{C}$ & -1.818305348957 & 1.172099306226 & -0.829607225562 \\
\hline S & -3.536604649772 & 1.617241550222 & -0.838228661704 \\
\hline $\mathrm{C}$ & -0.883490790558 & 2.177781107919 & -0.712766715057 \\
\hline $\mathrm{H}$ & -1.096191753256 & 3.228518605162 & -0.567156584887 \\
\hline $\mathrm{N}$ & 0.417175914146 & 1.800404862965 & -0.782930866068 \\
\hline O & 1.289093386492 & 2.906938352505 & -0.735025368197 \\
\hline C & 2.429800672725 & 2.668160459257 & -0.027508469636 \\
\hline $\mathrm{C}$ & 2.295606991500 & 1.718074395110 & 1.173049730180 \\
\hline $\mathrm{Cl}$ & 3.711076410528 & 0.738683883350 & 1.444890575768 \\
\hline 0 & 1.366668584849 & 1.755110782382 & 1.916919652865 \\
\hline O & 3.417005351807 & 3.300264035925 & -0.242493347060 \\
\hline $\mathrm{C}$ & 0.980558439834 & 0.605444549962 & -0.902365417697 \\
\hline $\mathrm{C}$ & -5.415218979414 & -1.930235902413 & 1.350047265672 \\
\hline $\mathrm{H}$ & -5.870662129126 & -2.767670211970 & 1.868496645926 \\
\hline $\mathrm{C}$ & -5.786639051274 & -1.635724474107 & 0.040059813712 \\
\hline $\mathrm{H}$ & -6.529197792015 & -2.243049835216 & -0.467129333303 \\
\hline C & -5.194122016759 & -0.565691818941 & -0.633020278628 \\
\hline $\mathrm{H}$ & -5.464338919483 & -0.343128259042 & -1.660434647011 \\
\hline $\mathrm{C}$ & -4.241707466562 & 0.216775992870 & 0.020508809263 \\
\hline C & -3.876329545943 & -0.065595467411 & 1.340646171974 \\
\hline $\mathrm{H}$ & -3.141394733125 & 0.555016480425 & 1.845269982917 \\
\hline C & -4.456611557611 & -1.147184365475 & 1.997404164903 \\
\hline $\mathrm{H}$ & -4.168589176685 & -1.371608364337 & 3.019345421618 \\
\hline $\mathrm{C}$ & 4.144863580979 & -2.937965378103 & -0.216136056757 \\
\hline $\mathrm{H}$ & 5.132200192727 & -3.286604983572 & 0.074567374154 \\
\hline $\mathrm{C}$ & 3.809401413269 & -2.817434511176 & -1.561355058149 \\
\hline $\mathrm{H}$ & 4.528668217189 & -3.065430669336 & -2.333484815246 \\
\hline $\mathrm{C}$ & 2.528510010186 & -2.376089039052 & -1.882899455792 \\
\hline $\mathrm{H}$ & 2.195354294531 & -2.262590842699 & -2.909482261082 \\
\hline $\mathrm{C}$ & 1.667737579194 & -2.073265695311 & -0.842353174291 \\
\hline $\mathrm{C}$ & 2.074593587659 & -2.218045379742 & 0.490529220096 \\
\hline $\mathrm{C}$ & 1.140705207016 & -1.888192635832 & 1.619897173200 \\
\hline $\mathrm{H}$ & 0.993657205939 & -0.804678151106 & 1.703191159391 \\
\hline $\mathrm{H}$ & 1.564526379482 & -2.249928013379 & 2.557015448649 \\
\hline $\mathrm{H}$ & 0.156701145594 & -2.339172528648 & 1.461083818693 \\
\hline $\mathrm{N}$ & 3.304471983076 & -2.652281692088 & 0.784977225123 \\
\hline
\end{tabular}


13 minor conf 0091

$\begin{array}{lc}S \bar{C} F \text { Done: } \bar{E}(\mathrm{RM} 06 \mathrm{X})=-2000.03585143 \quad \text { A.U. after } & 1 \text { cycles } \\ \text { Zero-point correction= } & 0.286226 \text { (Hartree/Particle) } \\ \text { Sum of electronic and zero-point Energies= } & -1999.749625 \\ \text { Sum of electronic and thermal Energies= } & -1999.725652 \\ \text { Sum of electronic and thermal Enthalpies= } & -1999.724708 \\ \text { Sum of electronic and thermal Free Energies= } & -1999.809374\end{array}$

\begin{tabular}{|c|c|}
\hline $\mathrm{C}$ & 1.078819334383 \\
\hline 0 & 2.226074317668 \\
\hline $\mathrm{C}$ & -0.116815258605 \\
\hline $\mathrm{H}$ & -0.053994633838 \\
\hline & -1.314560209540 \\
\hline & -2.898776522198 \\
\hline & -1.398005644595 \\
\hline & -0.204486512731 \\
\hline & -0.151159218802 \\
\hline & -1.195166160245 \\
\hline & -1.732292681741 \\
\hline $\mathrm{Cl}$ & -3.451232627688 \\
\hline 0 & -1.020372247555 \\
\hline 0 & -1.538447717577 \\
\hline C & 1.037994308457 \\
\hline $\mathrm{H}$ & 1.878735403375 \\
\hline & -1.822637709539 \\
\hline & -1.571538054231 \\
\hline $\mathrm{C}$ & -1.912806413164 \\
\hline $\mathrm{H}$ & -1.732884962796 \\
\hline $\mathrm{C}$ & -2.236555557428 \\
\hline $\mathrm{H}$ & -2.307615215077 \\
\hline C & -2.469023005202 \\
\hline$C$ & -2.384405851086 \\
\hline $\mathrm{H}$ & -2.570709644740 \\
\hline C & -2.058650186343 \\
\hline $\mathrm{H}$ & -1.992425607143 \\
\hline C & 5.820377233745 \\
\hline $\mathrm{H}$ & 6.779571220158 \\
\hline $\mathrm{C}$ & 5.348015926804 \\
\hline $\mathrm{H}$ & 5.929561337058 \\
\hline C & 4.114853583253 \\
\hline $\mathrm{H}$ & 3.682816205318 \\
\hline C & 3.433346175639 \\
\hline $\mathrm{C}$ & 3.971938035530 \\
\hline C & 3.237071087881 \\
\hline $\mathrm{H}$ & 2.469375301894 \\
\hline $\mathrm{H}$ & 3.941664454683 \\
\hline & 2.738786020720 \\
\hline & 5.158461695298 \\
\hline
\end{tabular}

0.173922387226

0.735848764061

0.902959216409

0.658088404064

0.723497246904

0.511401546251

0.431324947169

0.400240015584

0.493099900078

0.391339055797

1.011442613163

2. 280379433871

2. 506332627858

3.094518579319

0.672467738242

0.663891156561

0.731380106288

0.964920969158

1.091288042729

$-0.315957534567$

$-1.187379351908$

$-0.481385967579$

$-1.474562753335$

0.640856410197

1. 925066351155

2. 790194713217

2. 083952657848

3.080889820551

0.598314666456

0.466391882228

1.876013872582

2.751888684569

1.992361069454

2. 953860432686

0.825477011422

$-0.429987183052$

$-1.697306316677$

$-1.908658423101$

$-2.529108757334$

$-1.622893440230$

$-0.523348208089$ 
15 major conf 0006

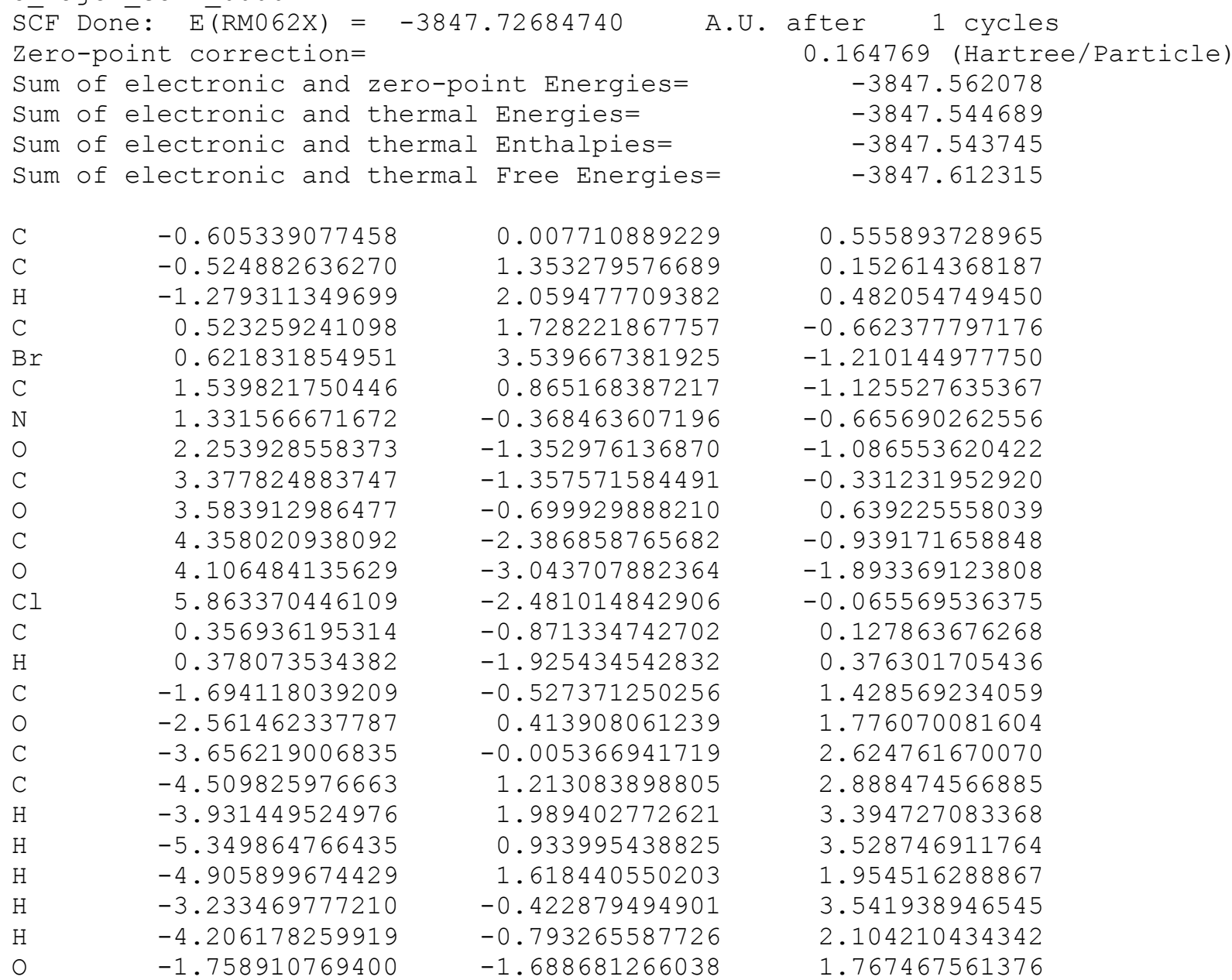


15 minor conf 0039

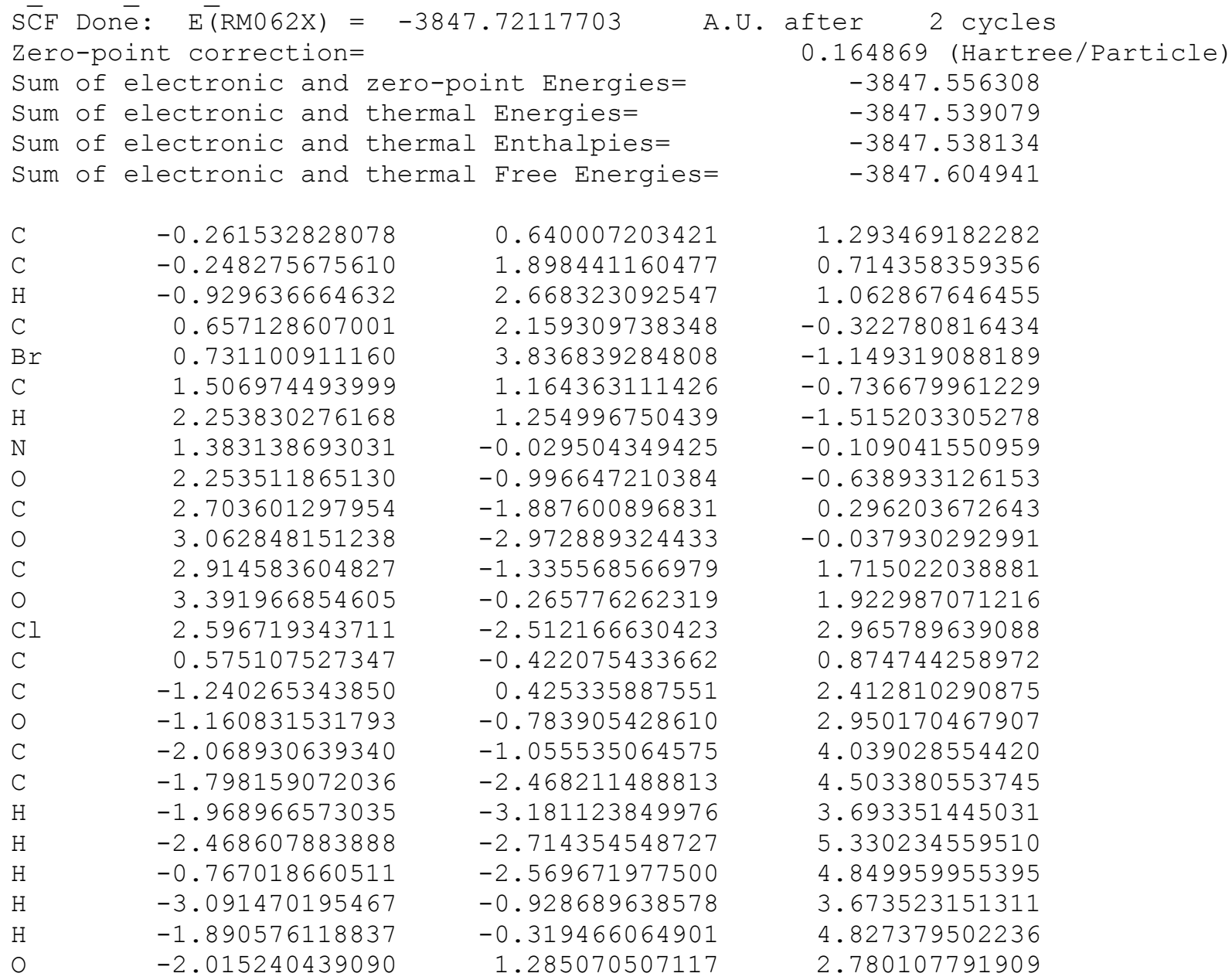


17 major conf 0056

$\begin{array}{lc}\mathrm{S} \bar{C} F \text { Done: } \bar{E}(\mathrm{RM} 62 \mathrm{X})=-1944.70310456 \quad \text { A.U. after } & 2 \text { cycles } \\ \text { Zero-point correction= } & 0.270633 \text { (Hartree/Particle) } \\ \text { Sum of electronic and zero-point Energies= } & -1944.432471 \\ \text { Sum of electronic and thermal Energies= } & -1944.410298 \\ \text { Sum of electronic and thermal Enthalpies= } & -1944.409354 \\ \text { Sum of electronic and thermal Free Energies= } & -1944.488935\end{array}$

$\begin{array}{lrrr}\mathrm{C} & 0.758427683163 & -0.010429484851 & -1.774865843911 \\ \mathrm{O} & 1.759251853514 & -0.003050427904 & -2.710400132816 \\ \mathrm{C} & 0.863343222181 & 0.878448949663 & -0.725505265926 \\ \mathrm{H} & 1.728838333454 & 1.530859205627 & -0.652125499874 \\ \mathrm{C} & -0.153370629414 & 0.917001088361 & 0.253199772308 \\ \mathrm{~S} & 0.030187065126 & 2.061489311698 & 1.592847674149 \\ \mathrm{C} & -1.216125964249 & 0.053089388588 & 0.123980718225 \\ \mathrm{H} & -2.049091290182 & -0.044570144625 & 0.807044972128 \\ \mathrm{~N} & -1.212979759221 & -0.746156949489 & -0.977228397400 \\ \mathrm{O} & -2.389370950551 & -1.518905014921 & -1.053779521274 \\ \mathrm{C} & -2.237989297775 & -2.702263255522 & -1.710328496948 \\ \mathrm{C} & -0.857663458009 & -3.373528661178 & -1.646387139180 \\ \mathrm{C} 1 & -0.437281389177 & -4.191115832803 & -3.136215365897 \\ \mathrm{O} & -0.244767018010 & -3.500659973943 & -0.633039433272 \\ \mathrm{O} & -3.191568545474 & -3.247415500942 & -2.176772307770 \\ \mathrm{C} & -0.350823086425 & -0.866852463569 & -1.971797360177 \\ \mathrm{C} & -3.708834976292 & 1.206017077763 & 4.073110623282 \\ \mathrm{H} & -4.595999038441 & 1.005508324839 & 4.665067034782 \\ \mathrm{C} & -2.533292030332 & 0.496281792569 & 4.319426961775 \\ \mathrm{H} & -2.502709798066 & -0.255479122640 & 5.101216240669 \\ \mathrm{C} & -1.390989041391 & 0.752288663945 & 3.562339831582 \\ \mathrm{H} & -0.471397855086 & 0.205446610417 & 3.746191419204 \\ \mathrm{C} & -1.434228947316 & 1.720026299756 & 2.555340268368 \\ \mathrm{C} & -2.608106689658 & 2.436561946194 & 2.308596471601 \\ \mathrm{H} & -2.627047467185 & 3.188146225804 & 1.525535213658 \\ \mathrm{C} & -3.746160968259 & 2.175074818278 & 3.070253683634 \\ \mathrm{H} & -4.659255345788 & 2.729504513502 & 2.880123842203 \\ \mathrm{C} & 3.296275360387 & -3.612581450237 & -4.093600115907 \\ \mathrm{H} & 3.714088231722 & -4.543427287936 & -4.462431434208 \\ \mathrm{C} & 3.023381653022 & -3.457032930053 & -2.734437641933 \\ \mathrm{H} & 3.225781329529 & -4.266994515269 & -2.040671393374 \\ \mathrm{C} & 2.486128110246 & -2.265237205576 & -2.253227308198 \\ \mathrm{H} & 2.274081633944 & -2.137958332706 & -1.195744773463 \\ \mathrm{C} & 2.221841861514 & -1.232547605616 & -3.151266304065 \\ \mathrm{C} & 2.485701104997 & -1.369413194137 & -4.509711202484 \\ \mathrm{H} & 2.265151916550 & -0.544909204563 & -5.179325256330 \\ \mathrm{C} & 3.026737626035 & -2.566604201721 & -4.976760503809 \\ \mathrm{H} & 3.233836560917 & -2.680811456804 & -6.036054029349 \\ & & & \end{array}$


17 minor conf 0071

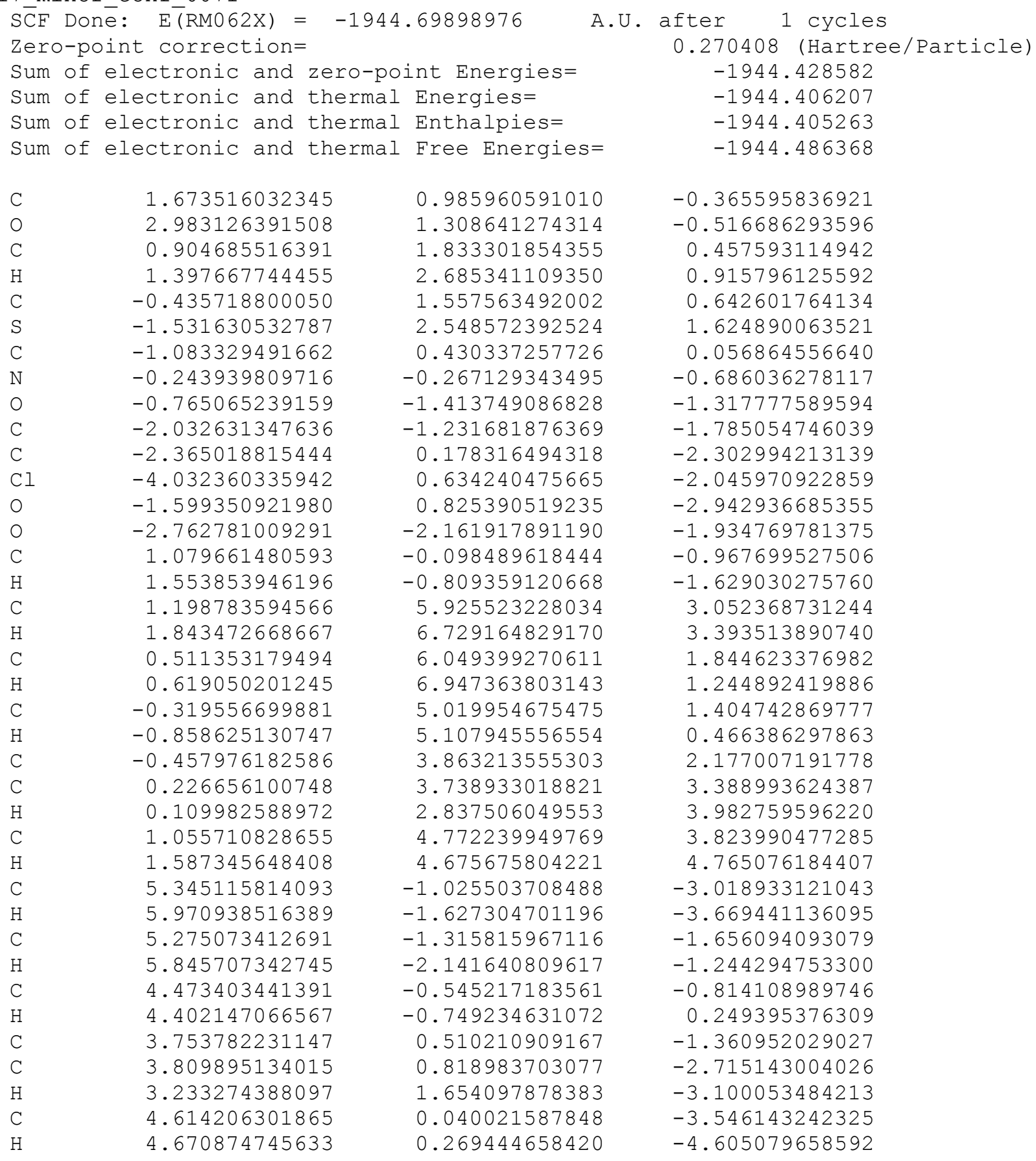




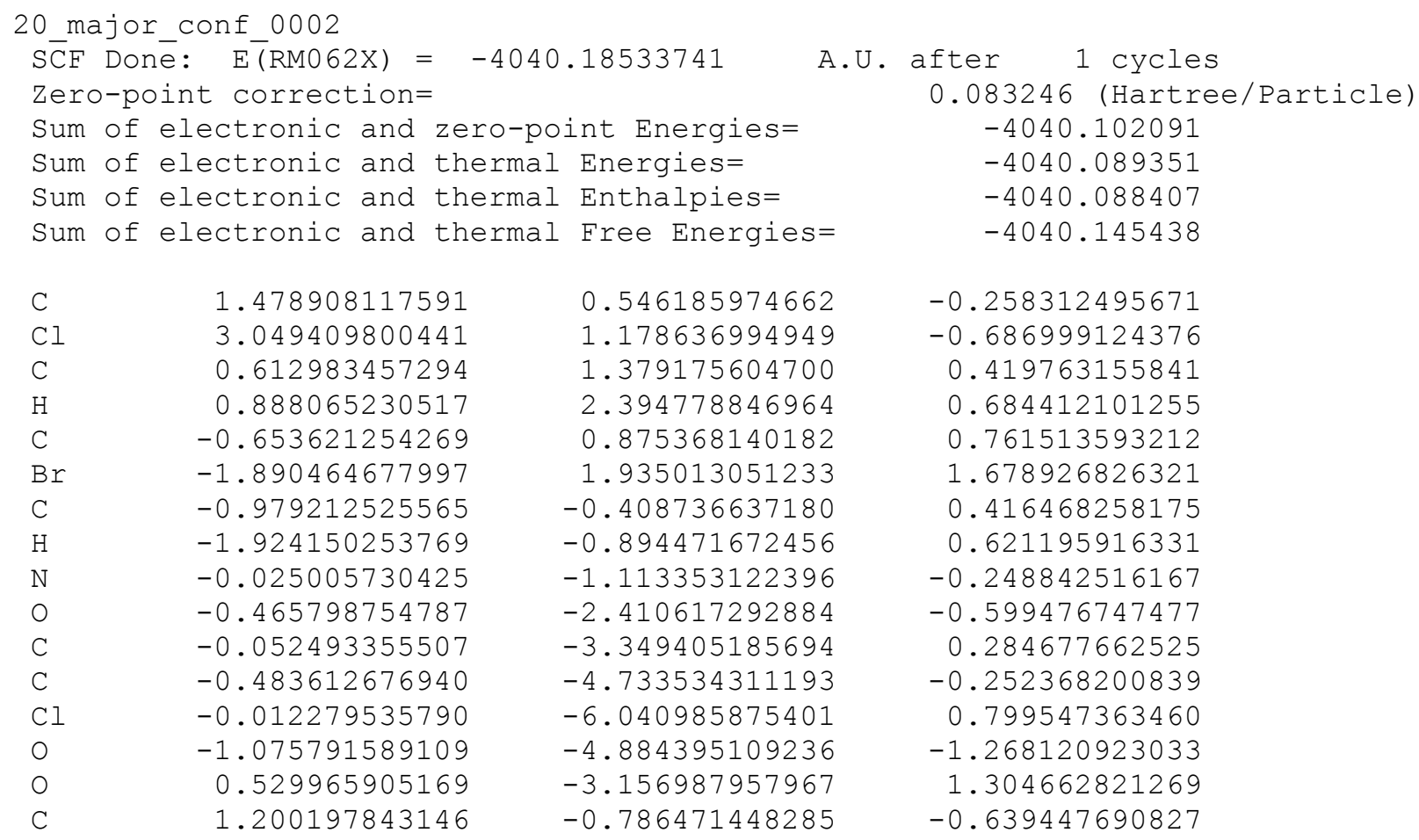




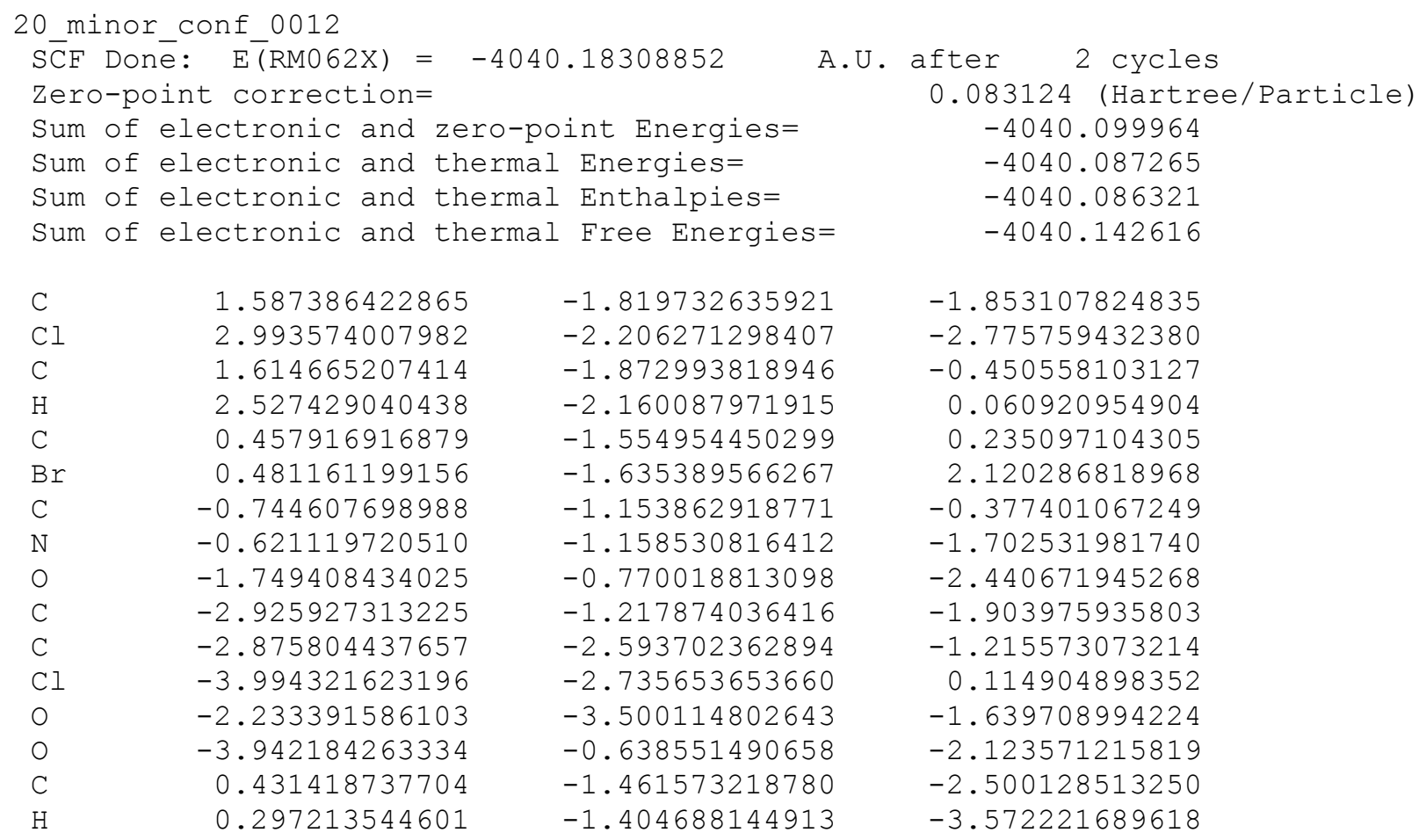

